\title{
Observations of Cool-Star Magnetic Fields
}

\author{
Ansgar Reiners \\ Georg-August-Universität \\ Institut für Astrophysik \\ Friedrich-Hund-Platz 1 \\ 37077 Göttingen \\ email: Ansgar.Reiners@phys.uni-goettingen.de \\ http://www.astro.physik.uni-goettingen.de/ areiners
}

Accepted on 20 February 2012

Published on 29 February 2012

(Revised on 29 March 2012)

\begin{abstract}
Cool stars like the Sun harbor convection zones capable of producing substantial surface magnetic fields leading to stellar magnetic activity. The influence of stellar parameters like rotation, radius, and age on cool-star magnetism, and the importance of the shear layer between a radiative core and the convective envelope for the generation of magnetic fields are keys for our understanding of low-mass stellar dynamos, the solar dynamo, and also for other large-scale and planetary dynamos. Our observational picture of cool-star magnetic fields has improved tremendously over the last years. Sophisticated methods were developed to search for the subtle effects of magnetism, which are difficult to detect particularly in cool stars. With an emphasis on the assumptions and capabilities of modern methods used to measure magnetism in cool stars, I review the different techniques available for magnetic field measurements. I collect the analyses on cool-star magnetic fields and try to compare results from different methods, and I review empirical evidence that led to our current picture of magnetic fields and their generation in cool stars and brown dwarfs.
\end{abstract}

This review is licensed under a Creative Commons Attribution-Non-Commercial-NoDerivs 3.0 Germany License. http://creativecommons.org/licenses/by-nc-nd/3.0/de/ 


\section{Imprint / Terms of Use}

Living Reviews in Solar Physics is a peer reviewed open access journal published by the Max Planck Institute for Solar System Research, Max-Planck-Str. 2, 37191 Katlenburg-Lindau, Germany. ISSN 1614-4961.

This review is licensed under a Creative Commons Attribution-Non-Commercial-NoDerivs 3.0 Germany License: http://creativecommons.org/licenses/by-nc-nd/3.0/de/. Figures that have been previously published elsewhere may not be reproduced without consent of the original copyright holders.

Because a Living Reviews article can evolve over time, we recommend to cite the article as follows:

Ansgar Reiners,

"Observations of Cool-Star Magnetic Fields",

Living Rev. Solar Phys., 8, (2012), 1. [Online Article]: cited [<date $>$ ], http://www.livingreviews.org/lrsp-2012-1

The date given as $<$ date $>$ then uniquely identifies the version of the article you are referring to. 


\section{Article Revisions}

Living Reviews supports two ways of keeping its articles up-to-date:

Fast-track revision A fast-track revision provides the author with the opportunity to add short notices of current research results, trends and developments, or important publications to the article. A fast-track revision is refereed by the responsible subject editor. If an article has undergone a fast-track revision, a summary of changes will be listed here.

Major update A major update will include substantial changes and additions and is subject to full external refereeing. It is published with a new publication number.

For detailed documentation of an article's evolution, please refer to the history document of the article's online version at http://www. livingreviews.org/lrsp-2012-1.

29 March 2012: Removed Figure 7 post-publication due to copyright restrictions. Springer did not grant permission to reuse material "in a work to be published on an Open Access Website".

Page 19: Removed Figure 7. 


\section{Contents}

1 Introduction $\quad 7$

2 Methodology of Magnetic Field Measurements 9

2.1 Zeeman effect . . . . . . . . . . . . . . . . . . . . 9

2.1.1 Absorption lines in a magnetic field . . . . . . . . . . . . . . . . . . . . 9

2.1.2 Polarization of Zeeman components . . . . . . . . . . . . . . 10

2.1 .3 The Stokes vectors . . . . . . . . . . . . . . . . . . 11

2.1.4 Reconstruction of stellar magnetic fields from Stokes vectors . . . . . . . . . 14

2.1 .5 Field, flux, and filling factor . . . . . . . . . . . . . . . . . . . 17

2.1.6 Equivalent widths . . . . . . . . . . . . . . . . . 17

2.1 .7 Doppler Imaging . . . . . . . . . . . . . . . . . . . . . . . . . . . . . . . . . . . . . . . . . . . . .

2.1 .8 Least Squares Deconvolution . . . . . . . . . . . . . . . . . . . . 22

2.2 Broad band polarization . . . . . . . . . . . . . . . . . . . . . 23

2.3 Indirect diagnostics . . . . . . . . . . . . . . . . . . . . . . . . . 24

3 Magnetic Field Measurements in Cool Stars $\quad 27$

3.1 Average magnetic fields from integrated light . . . . . . . . . . . . . . . 27

3.1.1 Sun-like stars . . . . . . . . . . . . . . . . . . . . . . . . . . . . . . . . 27

3.1 .2 M-type stars . . . . . . . . . . . . . . . . . . 29

3.1.3 Pre-main sequence stars and young brown dwarfs . . . . . . . . . . . . . . 33

3.2 Longitudinal fields and Zeeman Doppler maps from Stokes V . . . . . . . . . . . . 41

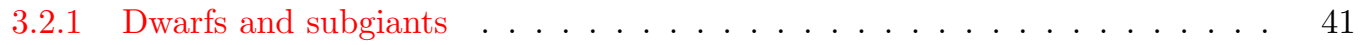

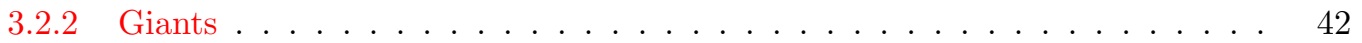

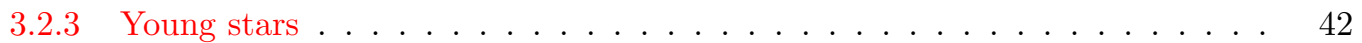

4 The Rotation-Magnetic Field-Activity Relation $\quad 45$

4.1 The dynamo at very low masses . . . . . . . . . . . . . . . . . . . . . . 47

4.2 Magnetism and $\mathrm{H} \alpha$ activity . . . . . . . . . . . . . . . . . . . . . . . . . . . . . . . . . . . . . . . . .

4.3 A posteriori knowledge about detectability of magnetic fields . . . . . . . . . . . . 49

$\begin{array}{llr}5 & \text { Equipartition } & 51\end{array}$

6 Geometries of Stellar Magnetic Fields $\quad 52$

7 Beyond Rotation $\quad \mathbf{5 7}$

7.1 A scaling law for saturated planetary and stellar dynamos . . . . . . . . . . . . 57

7.2 Brown dwarfs . . . . . . . . . . . . . . . . . . . . . . . . . . . . . . . . . . . . . . . . . 59

7.3 Fossil or dynamo fields in young low-mass stars . . . . . . . . . . . . . . . 59

8 Summary $r$

9 Acknowledgments $\quad 60$

$\begin{array}{lr}\text { References } & 61\end{array}$

\section{List of Tables}

1 Average magnetic fields from Stokes I measurements in sun-like stars. . . . . . . . 36

2 Average magnetic fields from Stokes I in M-dwarfs. . . . . . . . . . . . . . . . . 37 
3 Average magnetic fields from Stokes I in pre-main sequence stars and young brown dwarfs. . . . . . . . . . . . . . . . . . . . . 39

4 Magnetic field measurements not listed in Tables 1, 2, and 3. . . . . . . . . . 40

5 Longitudinal magnetic fields or Zeeman Doppler maps from Stokes V for dwarfs and subgiants. . . . . . . . . . . . . . . . . . . . . 43

$6 \quad$ Longitudinal magnetic fields or Zeeman Doppler maps from Stokes V for giants. . $\quad 44$

7 Longitudinal magnetic fields or Zeeman Doppler maps from circular polarization in pre-main sequence stars. . . . . . . . . . . . . . . . . . . . . . . 44 



\section{Introduction}

One of the reasons why the physics of magnetic fields are so attractive but poorly understood probably is that magnetic fields are invisible. Both, the detection of magnetic fields and the interpretation of field measurements connect a variety of research fields because magnetic effects are manifold and measurement processes involve a number of sophisticated techniques and unknowns. In the stellar context, magnetic fields are believed to be the reason why young stars can accrete material from their surrounding disk, they rule the evolution of angular momentum, and the stellar dynamo converts kinetic into thermal energy that appears in the many facets of stellar activity.

The observation of stellar magnetic fields is difficult because they are not directly visible, but also because we have only a very limited idea about the nature of the fields that may exist in stars other than the Sun. Because no measurement technique is capable of capturing the entire complexity of a stellar magnetic field, observations always only reveal that part of a magnetic field the observing strategy is specialized for - and in most cases it is not entirely clear what that is.

Our imagination of magnetic fields in cool stars rests on observations of the star we can observe in most detail - the Sun. Figure 1 shows an image of the solar surface from SOHO together with a magnetogram taken at the same time during solar maximum in 2001. Groups of cool spots appear where the magnetogram reveals regions of high fields. Interestingly, the fields appear in groups consisting of at least two areas in close proximity and of opposite polarity (for an overview of the solar magnetic field, see, e.g., Solanki et al., 2006). Obviously, such groups, if they exist, cannot be resolved in other stars where generally we can only observe the light integrated from the whole projected stellar surface.
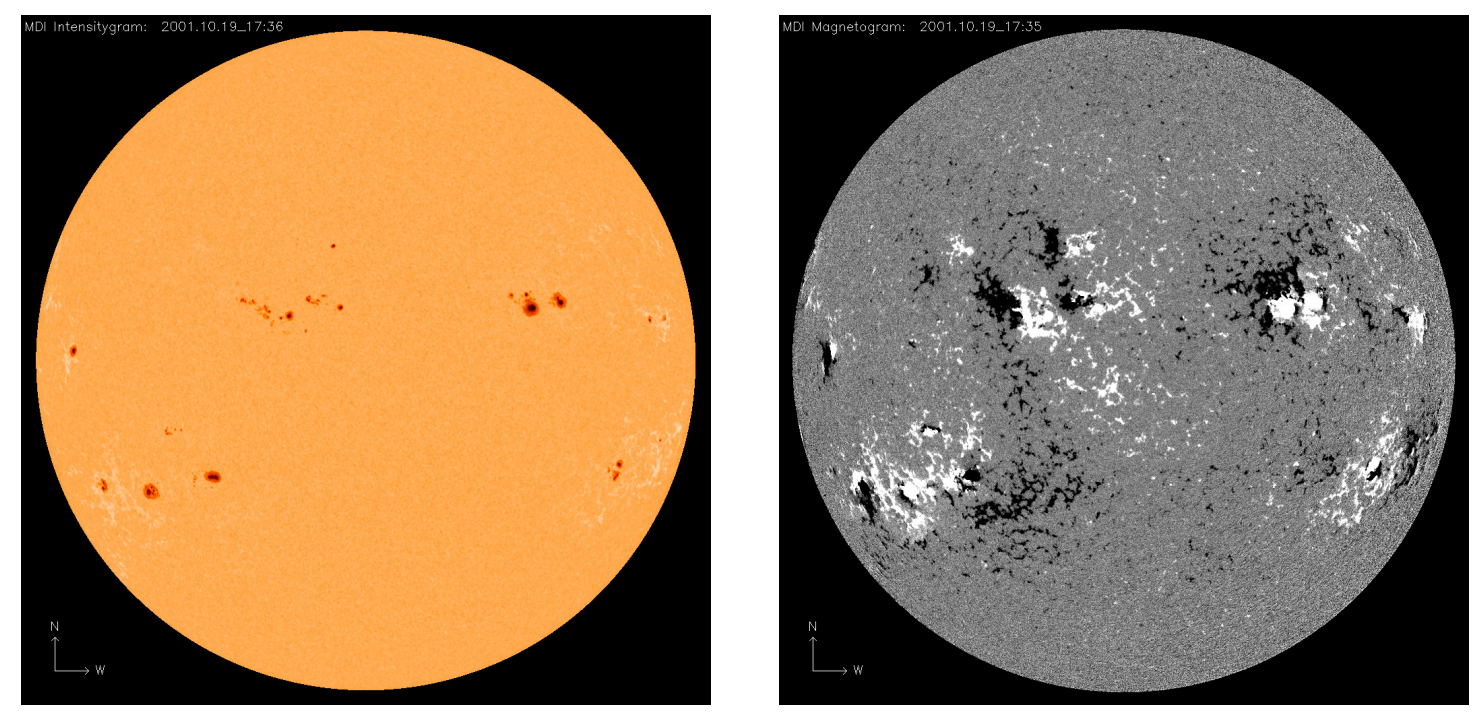

Figure 1: Images of the Sun taken with the SOHO (ESA \& NASA) satellite. Cool sunspots appear as dark areas in the visible light image (left). They correspond to magnetic areas seen as black or white areas in the magnetogram (right; black and white show magnetic areas of different polarity). The magnetic field is arranged in spot groups of opposite polarity.

Although it serves as reference for cool-star magnetism, the solar magnetic field is not at all easy to understand in all its details. The mean unsigned magnetic flux density on the solar surface is often reported to be on the order of $10 \mathrm{G}$ using Zeeman splitting diagnostics. However, Trujillo Bueno et al. (2004) reported average flux densities one order of magnitude higher employing a more sophisticated three-dimensional radiative transfer approach taking into account the con- 
sequences of the Hanle effect. The solar magnetic field is not the subject of this review, but the example shows how confusing even the magnetic field of the Sun can be if reduced to a single number. The reason for this is the wide range in strengths and scales that are probed using different methods.

Fortunately, not all stars have average magnetic flux densities as low as the solar one, and we absolutely can go out and look for fields that are stronger or have a more obvious observational signature than the solar field. Nevertheless, one has to keep in mind that all observations can only reveal the type of field they are sensitive to, and it is often more difficult to find out what that means than to actually carry out the observation. This article reviews the existing measurements of magnetic fields in cool stars. I define these to be stars with efficient convection in their surface layers, i.e., stars later than spectral type early F. Since F-type stars tend to be fast rotators, which makes a magnetic field detection even more difficult, a review on magnetic fields in cool stars essentially narrows down to stars cooler than the Sun. Magnetic field measurements are available for late-type dwarfs and also for some giants. One of the main motivations for investigating stellar magnetic fields is to understand the solar dynamo by assuming that the same mechanism works in other stars but runs with a different set of parameters. By studying magnetic fields in a sample of stars with different temperature, convective velocities, and rotation rates, one can hope to shed light on the fundamental mechanisms of a presumably universal cool-star dynamo mechanism.

A particularly interesting class of stars are cool stars of spectral type M. Covering the mass spectrum between $\sim 0.6$ and $0.1 M_{\odot}, \mathrm{M}$ dwarfs are the most frequent type of stars, which makes them very interesting by themselves. Furthermore, within this mass range, the stars can have very different physical properties rendering them very attractive targets for comparative studies. The transition from partly convective (sun-like) to fully convective stars happens in the M dwarf regime, probably around spectral type M3/M4. This area is in the center of interest for dynamo theory because the tachocline is believed to be the place where at least one important part of the solar dynamo is located. Furthermore, atmospheres of $\mathrm{M}$ dwarfs can be very different and both molecules and dust gain importance as the temperature drops toward late spectral types. It is important to understand how this influences magnetic field generation, and how the coupling between magnetic fields and stellar atmospheres changes. Towards even cooler objects, brown dwarfs are objects with masses below $0.08 M_{\odot}$ that are described as failed stars because they do not burn hydrogen in their core. Although they are not considered stars, their physical properties are very similar to low-mass stars, especially close to the surface. I will, therefore, include them in the discussion of stellar magnetic fields. 


\section{Methodology of Magnetic Field Measurements}

Magnetic fields are not directly visible. Their effects on the observable world are manifold, but all we can hope to measure are the consequences the presence of a magnetic field has on any properties that are accessible to observation. A particularly useful indicator of stellar magnetic activity, for example, is the non-thermal emission generated through magnetic heating (for a review, see Hall, 2008). Non-thermal emission is an example for indicators of magnetism that I will call indirect in the following. Indirect indicators require an additional mechanism to provide evidence for magnetic fields, and it is often difficult to entirely rule out alternative mechanisms as a source for its presence. For example, non-thermal emission may be generated by acoustic heating mechanisms so that the detectability cannot be translated into a magnetic field strength without further knowledge (Narain and Ulmschneider, 1996). Nevertheless, there is ample evidence that indirect indicators like chromospheric $\mathrm{Ca}$ or coronal X-ray emission are reliable tracers of magnetic flux density at least in sun-like stars (Schrijver et al., 1989; Pevtsov et al., 2003). For the following, an observable is called direct if its detection or amplitude necessarily implies the presence of a magnetic field.

The most successfully employed mechanism for direct detection of stellar magnetic fields is the Zeeman effect (Zeeman, 1897). Different approaches to use the Zeeman effect for magnetic field measurements and resulting field determinations are discussed and build the main part of the following chapters. Another mechanism that appears similar to the Zeeman effect and has been used in solar magnetic field measurements is the Hanle effect (Hanle, 1924). The Hanle effect describes how selective level population can be modified by a magnetic field (Landi Degl'Innocenti and Landolf, 2004; Trujillo Bueno, 2006). It can be used to measure tangled, very small-scale fields of up to a few hundred Gauss but requires a very detailed understanding of atomic level population and three-dimensional scattering processes (Trujillo Bueno et al., 2004). This level of detail cannot yet be achieved in stellar observations and the Hanle effect could so far not be used to detect magnetic fields in stars other than the Sun.

Observations of magnetically induced emission, i.e., indirect magnetic field diagnostics, provide a wealth of information on stellar magnetic activity that is often easier accessible than direct field measurements. For reviews on observations of coronal emission, chromospheric emission, and starspots the reader is referred to the reviews by Güdel (2002, 2004), Berdyugina (2005), and Hall (2008). In this article, the main consequences of indirect observations for our picture of stellar magnetism will be discussed only briefly in Section 2.3.

\subsection{Zeeman effect}

\subsubsection{Absorption lines in a magnetic field}

In this section, I will give a brief introduction on the basics of the Zeeman effect. More comprehensive discussions of the Zeeman effect and equations to calculate Zeeman splitting in stellar atomic absorption lines can be found, e.g., in Condon and Shortley (1963), Beckers (1969), Saar (1988), Landstreet (1992), Mestel and Landstreet (2005), and Donati and Landstreet (2009).

An atomic or molecular absorption or emission line is excited if electrons make a transition from one energy level to another. The energy of each level is altered in the presence of a magnetic field according to the vector product between the spin $(S)$ and orbital angular momenta $(L)$ of the electron, and the magnetic field vector (so-called LS coupling). Each energy level with total angular momentum quantum number $J$ splits into $(2 J+1)$ states of energy with different magnetic quantum numbers $M$. The difference between subsequent states of energy is proportional to $B g$ with $B$ the magnetic field and $g$ the Landé factor, the latter being a function of the energy level's orbital and spin angular momentum quantum numbers,

$$
g_{i}=\frac{3}{2}+\frac{S_{i}\left(S_{i}+1\right)-L_{i}\left(L_{i}+1\right)}{2 J_{i}\left(J_{i}+1\right)} .
$$


A dipole transition between two energy levels must obey the selection rule $\Delta M=-1,0,+1$, hence there are generally three groups of transitions between two energy levels. Spectral lines with $\Delta M=0$ are called $\pi$ components, spectral lines with $\Delta M=-1$ or +1 are called $\sigma_{\text {blue }}$ and

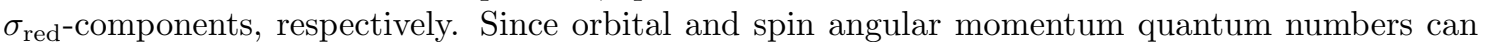
be different between the two energy levels, the Landé-factors of both levels can be different, and transitions between energy levels are not only a function of $M$ but depend on the Landé-factors of the energy states. The result is that each component consists of a group of transitions.

An often used quantity in the characterization of Zeeman splitting is the so-called effective Landé-factor, which is the average displacement of the group of $\sigma$-components with respect to line center. The effective Landé factor $g$ of a transition is a combination of Landé values of the two energy levels involved (Beckers, 1969),

$$
g=\frac{1}{2}\left(g_{u}+g_{l}\right)+\frac{1}{4}\left(J_{u}-J_{l}\right)\left(g_{u}-g_{l}\right)\left(J_{u}+J_{l}+1\right) .
$$

In principle, the effective Landé factor can be calculated from the energy level's individual Landé factors and Equation (2). For many transitions, however, LS-coupling is a poor approximation of the real situation leading to large errors in the calculation of individual $g_{i}$ values. In such cases, it can be more appropriate to measure $g_{i}$ in laboratory experiments (e.g., Reader and Sugar, 1975) and use Equation (2) to obtain more useful empirical effective Landé factors (Landi Degl'Innocenti, 1982; Solanki and Stenflo, 1985).

In summary, in the presence of a magnetic field, the transition energies of the $\sigma$-components are shifted according to the sensitivity of the transition (the Landé-factor $g$ ) and the strength of the magnetic field $B$. The energy perturbation depends on the energy level's quantum numbers, condensed in $g$, and the magnetic field, $B$. If we measure the energy shift in terms of wavelength shift $\Delta \lambda$ or as Doppler displacement $\Delta v$, the perturbation becomes a function of the initial wavelength of the transition, $\lambda_{0}$. The wavelength displacement of the $\sigma$ components is

$$
\Delta \lambda=46.67 g \lambda_{0}^{2} B
$$

with $\Delta \lambda$ in $\mathrm{m} \AA, \lambda_{0}$ in $\mu \mathrm{m}$, and $B$ in $\mathrm{kG}$. The average velocity displacement of the spectral line components can then be written as

$$
\Delta v=1.4 \lambda_{0} g B
$$

with $B$ in $\mathrm{kG}, \lambda_{0}$ in $\mu \mathrm{m}$, and $\Delta v$ in $\mathrm{km} \mathrm{s}^{-1}$. The typical Zeeman velocity displacement of a spectral line at visual wavelengths in the presence of a kG-field is on the order of $1 \mathrm{~km} \mathrm{~s}^{-1}$, which is somewhat smaller than the typical resolving power of a high-resolution spectrograph and the intrinsic line-width of stellar absorption lines. The Doppler displacement is proportional to the wavelength $\lambda_{0}$, which facilitates the detection of Zeeman splitting at infrared wavelengths compared to measurements in the visual.

\subsubsection{Polarization of Zeeman components}

The three groups of Zeeman components, $\sigma_{\text {blue }}, \sigma_{\text {red }}$, and $\pi$, are characterized by different magnetic moments, which means that the three Zeeman components have distinct polarization states. Furthermore, the actual intensity and polarization seen by an observer depends on the angle between the line of sight and the magnetic field at the atom. Figure 2 shows a simplified scheme of the splitting (left panel) and of the different observable polarization states (right) of the $\pi$ and $\sigma$ components: The $\pi$ component is not shifted in energy, it is always linearly polarized but is not observed if the line of sight is parallel to the magnetic field vector. The $\sigma$ components, on the other hand, are shifted according to the formulae above. They can be observed linearly or circularly polarized depending on the observer's view. If the line of sight is parallel (longitudinal field)

Living Reviews in Solar Physics

http://www. livingreviews.org/lrsp-2012-1 
to the magnetic field vector, both components are circularly polarized but in opposite directions. If the line of sight is perpendicular (transverse field) to the magnetic field, the $\sigma$-components are linearly polarized in the direction perpendicular to the polarization of the $\pi$-component.

It is important to realize that measurements of longitudinal and transerve fields as seen in circular and linear polarization, and also field measurements from unpolarized light are usually not identical to the real surface magnetic field because of the measuring principles discussed here. For the following, I will speak of longitudinal fields if magnetic fields are derived from circular polarization. This includes results from Stokes V magnetic maps, which combine observational information of longitudinal fields visible at different epochs.
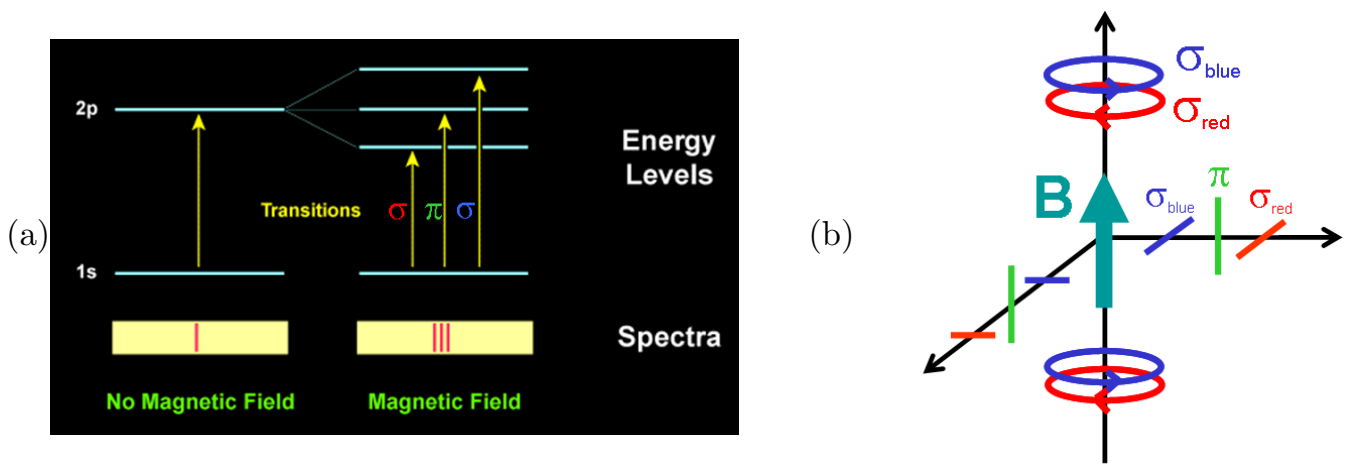

Figure 2: Schematic view of Zeeman splitting. (a) The upper level in the example is split into three levels producing three spectral lines that are separated. (b) Polarization of the $\pi$ and $\sigma$ components.

\subsubsection{The Stokes vectors}

For the characterization of a magnetic field, the measurement of intensity in different polarization states can be of great advantage. This is immediately clear from Figure $2 \mathrm{~b}$ since the different Zeeman components are polarized in a characteristic fashion. A commonly used system are the Stokes components I, Q, U, and V (Stokes, 1852) defined in the following sense:

$$
\begin{aligned}
& \mathrm{I}=\uparrow+\leftrightarrow \\
& \mathrm{Q}=\uparrow-\leftrightarrow \\
& \mathrm{U}=\nwarrow-\swarrow \\
& \mathrm{V}=\circlearrowleft-\circlearrowright
\end{aligned}
$$

Stokes I is just the integrated (unpolarized) light. Stokes Q and U measure the two directions of linear polarization, and Stokes V measures circular polarization. Note that Stokes Q and U are the differences between two linearly polarized beams with perpendicular directions of polarization. The components measured in Stokes $U$ are rotated by $45^{\circ}$ with respect to the components measured in Stokes Q; directions of $\mathrm{Q}$ and $\mathrm{U}$ are not defined in an absolute sense but require the definition of a frame of reference, in which polarization is measured. For a very readable introduction to Stokes vectors and alternative forms the reader is referred to Tinbergen (1996).

Stokes vectors are useful in astronomy because perpendicular circular and linear polarization states can be measured with relatively straightforward instrumentation. The representation of astronomical polarization measurements is usually done in terms of Stokes vectors. The problem we are concerned with, however, is under what circumstances the magnetic field of a star can be recovered from measurements of the Stokes vectors. If solar magnetic fields are a good example for other stellar magnetic fields, we can expect that they often show up in groups of different polarity. 
This is a problem to measurements in Stokes $\mathrm{V}$ because equal amounts of polarized light with opposite polarization simply cancel out and become invisible. In Stokes Q and U, on the other hand, regions with magnetic field vectors pointing into directions perpendicular to each other will cancel. Thus, magnetism on a star with one magnetic region on the eastern limb and another one of an identical field strength and geometry on the northern limb will show no linear polarization and be invisible to Stokes Q and U measurements.

In reality, stellar magnetic fields can be expected to be very complicated structures with a continuum of field strengths and orientations. Therefore, we can in general not expect to resolve classical splitting patterns with three groups of Zeeman components, because magnetic field strengths and, thus, the velocity displacement of the $\sigma$-components will be continuous. Finally, the visible surface of a star is not a flat disk but one half of a sphere and even the academic case of a completely radial (non-potential) field has vector components that would be observed under viewing angles between $0^{\circ}$ and $90^{\circ}$. It is, therefore, a formidable task to measure magnetic fields and their geometries in stars that are spatially entirely unresolved.

For a first estimate of the signals that may be expected from Stokes measurements in sunlike stars, we can take a look at sunspot data. In the center of a sunspot with $B=2200 \mathrm{G}$, the polarization of spectral lines at around $630 \mathrm{~nm}$ is on the order of $10 \%$ in Stokes Q and U, and about $20 \%$ in Stokes $\mathrm{V}$ if measured at very high spectral resolving power $(R=200000)$ (Lites, 2000). Observations of spatially unresolved sun-like stars will obviously not reach that level because of canceling effects and lower spectral resolution. Piskunov and Kochukhov (2002) calculated the four Stokes parameters in profiles at $\lambda=615 \mathrm{~nm}$ for a star with a dipolar magnetic field configuration with a field strength of $8 \mathrm{kG}$ at a spectral resolving power of $R=100000$. This model star is probably not very similar to a low-mass star, but the example nicely shows what level of polarization can be expected in an extreme case of a very strong and organized magnetic field. The signal from disk-integrated observations reaches maximum values of $0.5 \%$ in Stokes Q and U, and 5\% in Stokes V. Thus, if magnetic fields are to be detected in all four Stokes parameters, extremely high data quality is required. At visual wavelengths, linear polarization observations must have signal-to-noise ratios of roughly 1000 while circular polarization perhaps is relaxed by a factor 10, approximately. For similar magnetic field measurements in sun-like stars, the requirements are likely higher by at least one order of magnitude.

A series of very high quality stellar measurements in all four Stokes vectors of magnetic Ap and Bp stars was presented by Wade et al. (2000). Again, no comparable measurements are available for sun-like stars, but hotter stars with very strong fields may serve as guideline for our expectations of polarization in cool star observations. Wade et al. (2000) show that in magnetic Ap and Bp stars, circular polarization detected in strong, magnetically sensitive lines is typically around $1-2 \times 10^{-2}$ while linear polarization is a factor $10-20$ lower. Typical fields in cool stars are probably much weaker so that polarization can be expected to be a lot weaker than this, too. Recently, Kochukhov et al. (2011) presented the first detection of linearly polarized spectra in cool stars. In an active K-dwarf, they detected circular polarization at a level of $5 \times 10^{-5}$, and linear polarization of roughly a factor of 10 weaker.

In order to demonstrate the visibility of magnetic fields in the Stokes parameters and the canceling effects of spot groups with opposite polarization (as observed on the Sun), Figures 3 and 4 show some basic simulations of line profiles from magnetic regions in the Stokes parameters. The left panel visualizes the "geometry", which is actually not a geometry of some real stellar magnetic field, but nothing else than two areas of radial magnetic fields put on a flat surface, where the spherical shape of a star has not been taken into account in this example. It should be emphasized that on a real, spherical star, cancellation effects would not be as obvious as in this simplistic example and some net flux would usually remain. This is even more important in the case of rotating stars where opposite polarization of magnetic regions with different (local) radial velocities would not lead to complete flux cancellation.

Living Reviews in Solar Physics

http://www. livingreviews . org/lrsp-2012-1 
The magnetic regions in this toy model as shown in Figure 3 are observed with the field direction perpendicular to the line of sight, i.e., observations of the transverse field. Figure 4 shows the same cases for observations of magnetic regions observed with the field direction parallel to the line of sight, i.e., the longitudinal field. In the first case of transverse field orientation, no circular polarization is visible at all. For longitudinal field observations, linear polarization is invisible. Spectral resolving power is set to $R=100000$, the model line is a fictitious Fe line at rest wavelength $600 \mathrm{~nm}$, thermally broadened according to a temperature of $4000 \mathrm{~K}$. Broadening due to turbulence and rotation are not taken into account.

\section{"Geometry"}

Stokes I
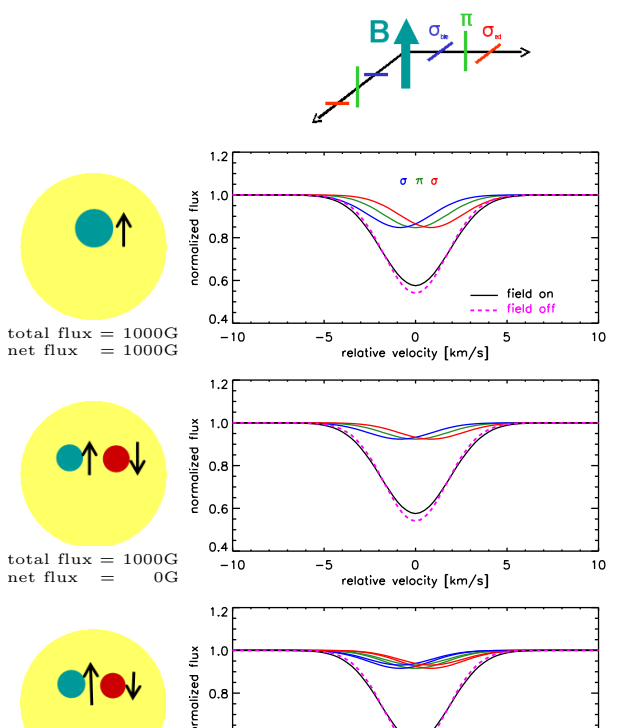

total flux $=1000 \mathrm{G}$

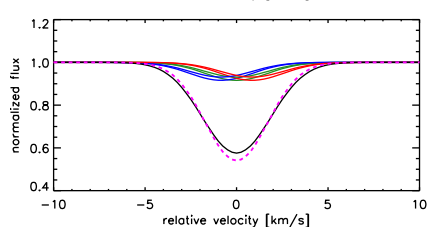

Stokes V
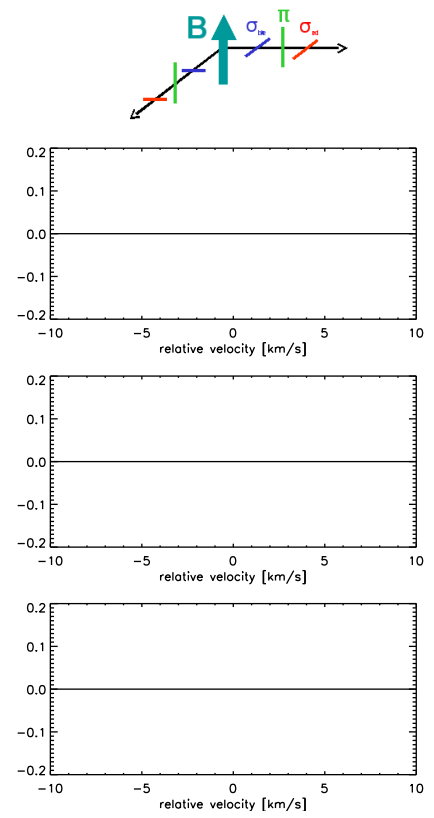

\section{Stokes Q or U}
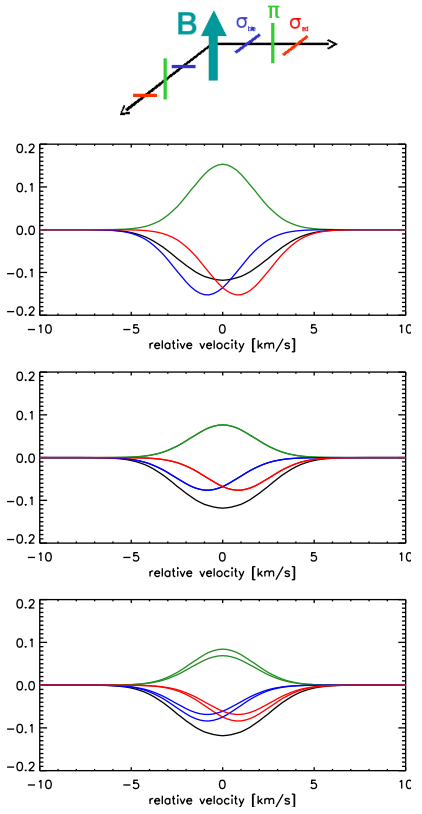

Figure 3: Three examples of simplified field geometries and their signals in Stokes I, V, and Q or U if the field is perpendicular to the line of sight (transverse field). Blue, green, and red lines show the line profiles of individual Zeeman components $\sigma_{\text {blue }}, \pi$, and $\sigma_{\text {red }}$, respectively. The black line is the sum of the three, that means the line that will be observable. In the Stokes I panel, the magenta line shows how the line would appear with zero magnetic field. Rotational Doppler effects are ignored in these examples.

The first example in the top row of both figures is a simple magnetic field region with only one polarity; total field strength and signed "net" field both are $1000 \mathrm{G}$ in this example. Stokes I exhibits very little broadening that is difficult to detect. There is a difference between the two directions of observation since for the longitudinal field (Figure 4), the $\pi$ component does not appear. Linear polarization of the transverse field (Figure 3) and circular polarization of the longitudinal field (Figure 4) are on the order of $10 \%$. Note that the direction of the Stokes V signal indicates the orientation of the magnetic field vector. In the second row, two magnetic regions, each with only half the size as in the first example are observed. Both regions have the same absolute field strength and area, but opposite polarity. The Stokes I signal is identical to the first example (the individual components are weaker but there are twice as many). The same is true for the linear polarization signal in Stokes Q and U because a shift in the polarization direction of $180^{\circ}$ has the same signal as the original one. The signal in Stokes V, however, entirely vanishes (for all observing angles) because the net (signed) field of this configuration is exactly zero; any field 

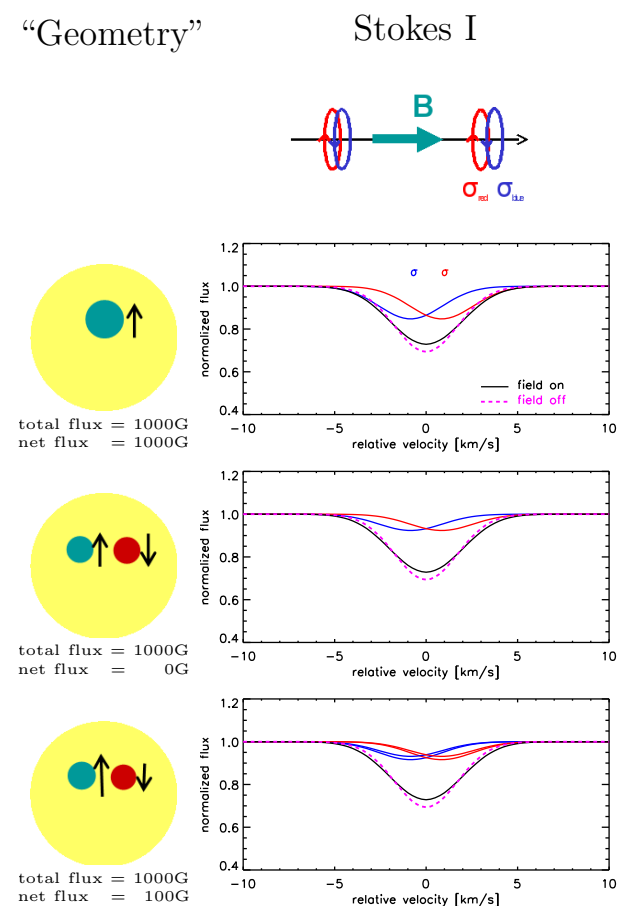
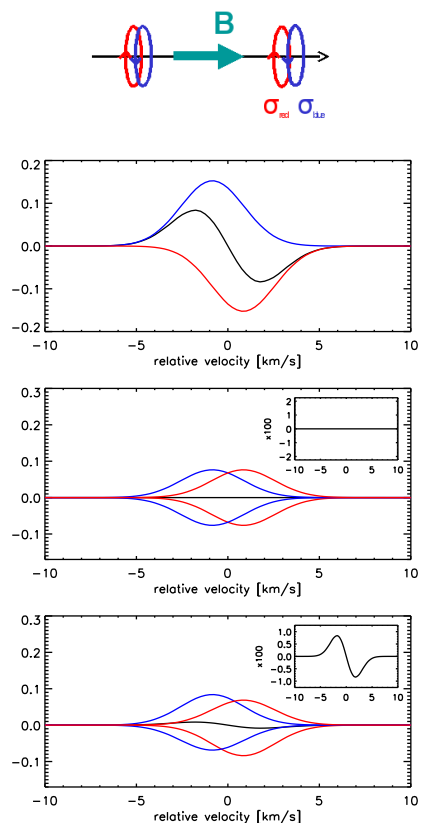

Stokes Q or U

Figure 4: Three examples of simplified field geometries and their signals in Stokes I, V, and Q or U if the field is tangential to the line of sight (longitudinal field). Colored lines like in Figure 3. Rotational Doppler effects are ignored in these examples.

strength in this canceling configuration is invisible to Stokes V. The last row in Figures 3 and 4 show the case of two magnetic areas with slightly different sizes; the total field is still $1000 \mathrm{G}$, but here the net field is $100 \mathrm{G}$. Again, Stokes I, and Q and U are the same as in the examples above. Because of the non-vanishing net field, the amplitude in Stokes $\mathrm{V}$ is now different from zero at ca. 1\%. In Figure 3, linear polarization always provides a strong signal because opposite magnetic field polarities do not cancel out. In a situation with two spots located at relative polarization of $90^{\circ}$ to each other, linear polarization would completely cancel, too. As mentioned above and will be discussed again in Section 2.1.7, Doppler shifts on a rotating star add valuable signal to polarimetric measurements. Since real stars have usually non-zero rotation, most cool stars show non-zero magnetic features visible in polarimetric measurements.

\subsubsection{Reconstruction of stellar magnetic fields from Stokes vectors}

The few examples shown in Figures 3 and 4 demonstrate the principal sensitivity of the four Stokes vectors to magnetic fields and their configurations. In spatially resolved regions on the solar surface, measurements of polarization provide relatively well-defined information on the magnetic field (at least if compared to the case in other stars). In other stars, however, we do not quite know what kinds of fields to expect. The average flux density on the Sun is only on the order of a few $\mathrm{G}$ and remains undetectable in observations of integrated solar light. Slowly rotating stars of a comparable activity level probably have fields as weak as the solar one. On the other hand, the magnetic geometry of more rapidly rotating and, hence, more active stars is entirely unknown and may not be very similar to the solar case.

A major difficulty in measuring stellar Zeeman splitting is the small value of $\Delta v$ compared 
to other broadening agents like intrinsic temperature and pressure broadening, and rotational broadening. In a kG-magnetic field, typical splitting at optical wavelengths is of the order $1 \mathrm{~km} \mathrm{~s}^{-1}$, which is well below intrinsic line-widths of several $\mathrm{km} \mathrm{s}^{-1}$ and also below the spectral resolving power of typical high-resolution spectrographs. Thus, individual components of a spectral line can normally not be resolved even if the star only had one well-defined magnetic field component. Real stars, however, can be expected to harbor a magnetic field distribution that is much more complex than this. Thus, even if spectral lines were intrinsically very narrow and spectral resolving power infinitely high, we would expect the Zeeman-broadened lines to look smeared out since in our observations we integrate over all magnetic field components on the entire visible hemisphere.

Stellar activity manifests itself in magnetic regions that can be darker than the quiet photosphere (e.g., spots) or brighter (e.g., faculae). The contribution of a surface region to an observed spectral line depends on its intensity contrast and local opacity while average field densities in active regions like spots or faculae are known to be systematically different from each other. This implies that regions of different field strenghts are systematically weighted in their contribution to the observed Zeeman pattern, and that the choice of diagnostic is very important for the field density measured.

Another point that becomes immediately clear is that the geometric interpretation of Zeeman splitting on an unresolved stellar disk can be arbitrarily complex, no matter if polarized or unpolarized light is used. In addition to the ambiguity between magnetic field strength and the fraction of the star being occupied with magnetic fields (which includes our ignorance about the number and distribution of magnetic components), the signature of a magnetic field region in stellar spectra depends on the angle between the magnetic field lines and the line of sight. In reality, a continuous distribution of angles can be expected because field lines are probably bent on the stellar surface, and because the stellar surface is spherical. As a result, even geometrically relatively simple field distributions will lead to highly complex splitting patterns. If the star is rotating at significant speed, as most active stars probably do, that pattern again depends a lot on the time a star is observed. This, in turn, can be utilized to reconstruct the geometry of the magnetic field by observing the variation of the observed spectra with rotation.

There are two basically quite different ways to gather information about stellar magnetic fields:

1. Measure the integrated scalar, unsigned magnetic field (Stokes I).

2. Measure the magnetic vector field.

The most promising way, clearly, to obtain information about the magnetic field is to determine simultaneously the integrated field and its vector components. Observationally, however, there are important differences between measurements in Stokes I (integrated flux measurements) and measurements in polarized light, so that in practice both parts are often done separately.

\section{Integrated field measurements}

The value of the integrated magnetic field strength can be derived from observations in Stokes I. Such observations can be carried out with every high-resolution spectrograph and do not require polarization optics. Stokes I measurements are sensitive to the entire magnetic field on the star, independent of field geometry and canceling effects. A simultaneous measurement of Stokes I is, therefore, always helpful in order to determine the fraction of a magnetic field that may be invisible to polarized light measurements.

Unfortunately, in a measurement of Zeeman splitting in Stokes I one faces the difficulty to disentangle the effect of Zeeman broadening from all other broadening agents. This requires precise knowledge of the spectral line appearance in the absence of a magnetic field. This task requires extremely good knowledge about spectral line formation, velocity fields, and the temperature distribution on the star. Signatures of cool spots or differential rotation, for example, can be very 
similar to Zeeman splitting patterns in integrated starlight. The amplitude of Zeeman spitting due to a strong magnetic field (e.g., $1000 \mathrm{G}$ ) is very subtle in sun-like stars observed at visual wavelengths because intrinsic line width, surface velocity, and typical instrumental resolution are of the same order as Zeeman broadening. This implies that the detection of magnetic fields lower than $\sim 1 \mathrm{kG}$ is extremely difficult at visual wavelengths (see Section 3.1). Thus, stellar Stokes I measurements are typically not sensitive to magnetic fields lower than a few hundred Gauss. The degeneracy between Zeeman splitting and other broadening agents is lifted at longer wavelengths, hence infrared observations have much higher sensitivity to magnetic fields. Unfortunately, only very few high-resolution infrared spectrographs exist today but more and more measurements are being reported (Section 3.1).

The Zeeman splitting pattern in surface-integrated starlight is the sum of Stokes I patterns from the entire stellar surface. The absorption line from a star is very different from a sunspot observation in which individual components from relatively well-defined magnetic regions can be visible. The line broadening pattern in Stokes I depends on the magnetic field strength of the individual components, the strongest fields are visible in the components responsible for the widest line wings. The fractional area of the surface filled with magnetic fields (filling factor) and the weight of individual surface features in the final line profile are parameters that are hidden in the line profile shape and are degenerate with respect to each other. The information on the field distribution and the contribution of individual magnetic areas is, therefore, very limited in observations of Stokes I alone. Another limitation of Stokes I measurements became visible in observations of the solar magnetic field using the Hanle effect (see above). These measurements revealed that the Sun harbors a field that is not of $10 \mathrm{G}$ but more of $100 \mathrm{G}$ strength. It is unclear whether a similar difference (either in absolute or relative units) would also appear if stars with much higher field strengths are observed, but it clearly shows that Stokes I measurements have difficulties capturing the entire magnetic flux but can mainly provide a lower limit.

\section{Reconstruction of the magnetic vector field}

Observations in Stokes V, Q, or U are sensitive to the magnetic field vector, not only to the unsigned field. This provides information about the direction of the magnetic field that is not accessible to Stokes I measurements. The signal of a non-polarized spectral line is zero in Stokes V, Q, and U. This means that the problem of disentangling Zeeman splitting from other line broadening mechanisms does not exist, and the method is much more sensitive to small field values ( $1 \mathrm{G}$ and below). A problem is, however, that the signal seen in polarized light is only the "net" magnetic field; regions of opposite polarity cancel out in Stokes V and magnetic fields at $90^{\circ}$ orientation cancel out in Stokes Q and U. Therefore, depending on what observing technique is used, an arbitrary large magnetic field may be hidden on the stellar surface without any signal in Stokes V or Stokes Q and $\mathrm{U}$ alone. The problem is more severe for circular polarization because the $\pi$ components are not detected here.

It has been shown that the magnetic field distribution of a star can be reconstructed in great parts from simultaneous observations of all four Stokes parameters (Kochukhov and Piskunov, 2002; Kochukhov et al., 2010). Successful reconstruction requires that the star is observed over an entire rotation period for two reasons: 1) to reconstruct the surface field hemisphere, the star needs to be seen from different sides (note that if the star is seen under high inclination angles, the invisible part close to the hidden pole always remains undetectable); 2) at different phases, the angles between the magnetic field lines and the line of sight vary with the result that field components that may have canceled when observed at disk center, can become visible when observed close to the limb. The spatial resolution of magnetic field reconstructions depends on the frequency of observations during stellar rotation and on intrinsic line broadening (all Stokes components are subject to line broadening). Typically, a resolution element has a size of ten or

Living Reviews in Solar Physics

http://www. livingreviews.org/lrsp-2012-1 
several ten degrees on the stellar surface. Kochukhov and Piskunov (2002) showed that using only a subset of Stokes vectors leads to ambiguities that should be interpreted with great caution. Unfortunately, measurements of linear polarization are extremely challenging in cool stars because of the low polarization signal so that typically only Stokes I and (sometimes) Stokes V are available (see Section 3.2.1). Zeeman broadening in Stokes I is very subtle at least at visual wavelengths where most available spectrographs operate, and Stokes V, Q, and U measurements are both difficult to acquire and exhibiting subtle Zeeman signals. The observational difficulties obtaining all four Stokes components led to the practice that in cool stars in the past usually either Stokes I or Stokes V alone were investigated.

\subsubsection{Field, flux, and filling factor}

In general, a stellar surface may be covered with a homogenous field of one particular field strength, or it can be covered with several magnetic areas of different field strength. One example is a surface of which $50 \%$ is covered with a field of strength $B$. If the other $50 \%$ of the surface has no magnetic field, the average field is $B f=B / 2$ with filling factor $f=0.5$. An important consequence of the fact that individual Zeeman-components are usually not resolved is the degeneracy between magnetic field $B$ and filling factor $f$. A strong magnetic field covering a small portion of the star looks similar to a weaker field covering a larger portion of the star. An often used way around this ambiguity is to specify the value $B f$, i.e., the product of the magnetic field and the filling factor; if more than one magnetic component is considered, $B f$ is the weighted sum over all components. Products of $B$ with some power of $f$, for example $B f^{0.5}$ or $B f^{0.8}$ are often considered because they seem to be better defined by observations (see Gray, 1984; Saar, 1988; Valenti et al., 1995). One important point to observe is that $B f$ is often called the "flux" - because it is the product of a magnetic field and an area - but it has the unit of a magnetic field. In fact, the term flux is very misleading since: 1) with $f$ specifying a relative fraction of the stellar surface, $B f$ is really the average flux density that is identical to the average unsigned magnetic field on the visible stellar surface, i.e., $B f \equiv\langle B\rangle$; and 2) the total magnetic flux of two stars with the same values of $B f$ can be extremely different according to their radii because the actual flux is proportional to the radius squared, $\mathcal{F} \propto B f r^{2}$. As a consequence, the value $B f$ will be much lower in a young, contracting star compared to an older (smaller) one if flux is conserved.

A related source of confusion is the difference between the signed magnetic field (or flux), and the unsigned values or the square of the fields (used to calculate magnetic energy). With Stokes I, both polarities produce the same signal and the total unsigned flux is measured. This implies that Stokes I carries only partial information about field geometry, but it also means that Stokes I always probes the entire magnetic flux of the star (see above). On the other hand, Stokes V can provide information on the sign of the magnetic fields, but this comes with the serious caveat that opposite magnetic fields cancel out and can become invisible to the Stokes V signal. Thus, results on $B f$ from Stokes $\mathrm{V}$ measurements can be much lower than Stokes I measurements.

\subsubsection{Equivalent widths}

Shifting of the $\sigma$-components to either side of the line center leads to broadening of the spectral line and, in general, to a flattening of the line core (see Figure 3). An interesting effect can be used to measure magnetic fields if lines that are saturated are used, i.e., lines that have equivalent widths smaller than the sum of the individual $\pi$-and $\sigma$-components. If such a saturated line is split in the presence of a magnetic field, the core depth of the line will remain at approximately constant level while the line grows wider (see Figure 5). As a result, the equivalent width of a saturated, magnetically sensitive line will grow with magnetic field strength.

Basri et al. (1992) introduced a method to detect cool star magnetic fields searching for enhanced equivalent widths of Zeeman-sensitive absorption lines. As in other work searching for 

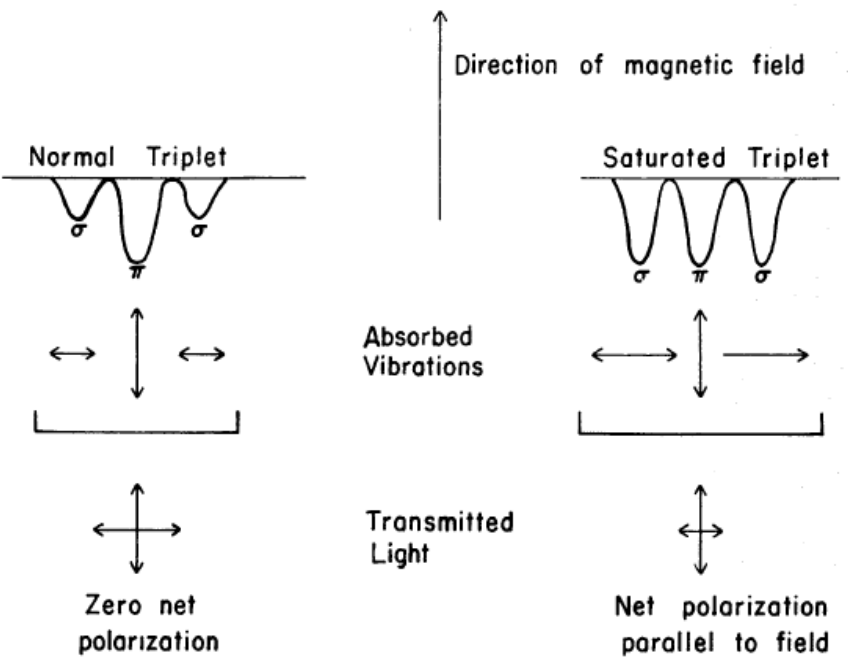

Figure 5: The net polarization of a weak line is zero, and its equivalent width remains constant if a magnetic field is applied. In contrast, the net polarization of a saturated line in a transverse magnetic field is nonzero, and the equivalent width of a saturated line becomes larger in a magnetic field (from Mullan and Bell, 1976, after Leroy, 1962; reproduced by permission of the AAS).

Zeeman splitting in Stokes I observations, they carefully modeled polarized line transfer and compared the appearance of Zeeman sensitive to Zeeman insensitive lines. The advantage of the equivalent width method is that equivalent widths are more easily measured than the subtle differences in line shape, in other words, information from several spectral bins within one spectral line is extracted into one number that can be measured more accurately. Nevertheless, the method cannot lift degeneracies between magnetic field strength (times filling factor) and other features like starspots or uncertainties in the model atmosphere; the equivalent width method can only make existing differences in the lines easier detectable.

The variation of line equivalent widths can be monitored over time. If one assumes that variations occur because of varying visible magnetic field strength, spectroscopic time series can be used to obtain information about the surface distribution of co-rotating magnetic regions (see also next section). This method was used for example by Saar et al. $(1992,1994$ c) for Stokes I magnetic surface imaging.

\subsubsection{Doppler Imaging}

In addition to measuring the average magnetic field on a star, signed or unsigned, the Doppler shift of individual features carries information about the geometry of the stellar surface. Doppler Imaging exploits the correspondence between wavelength position across a rotationally broadened spectral line and spatial position across the stellar disk to reconstruct surface maps of rotating stars (Vogt and Penrod, 1983); the method goes back to work by Deutsch (1958), Falk and Wehlau (1974), and Goncharskii et al. (1977). Spatial resolution of the maps depends on the rotation velocity of the star and the sampling frequency at which spectra are taken, among other factors. It has been used very successfully to reconstruct temperature maps of cool stars (see, e.g., Strassmeier, 2002) and abundance maps of hotter stars (e.g., Kochukhov et al., 2004). Zeeman Doppler Imaging (ZDI) follows the same approach but investigating polarized light (Semel, 1989). As the star is observed at different phases, the magnetic field vectors are observed under different projection

Living Reviews in Solar Physics

http://www. livingreviews.org/lrsp-2012-1 
angles leading to characteristic signatures in polarized light; field components that may be invisible at one phase can have large Stokes parameters at other phases.

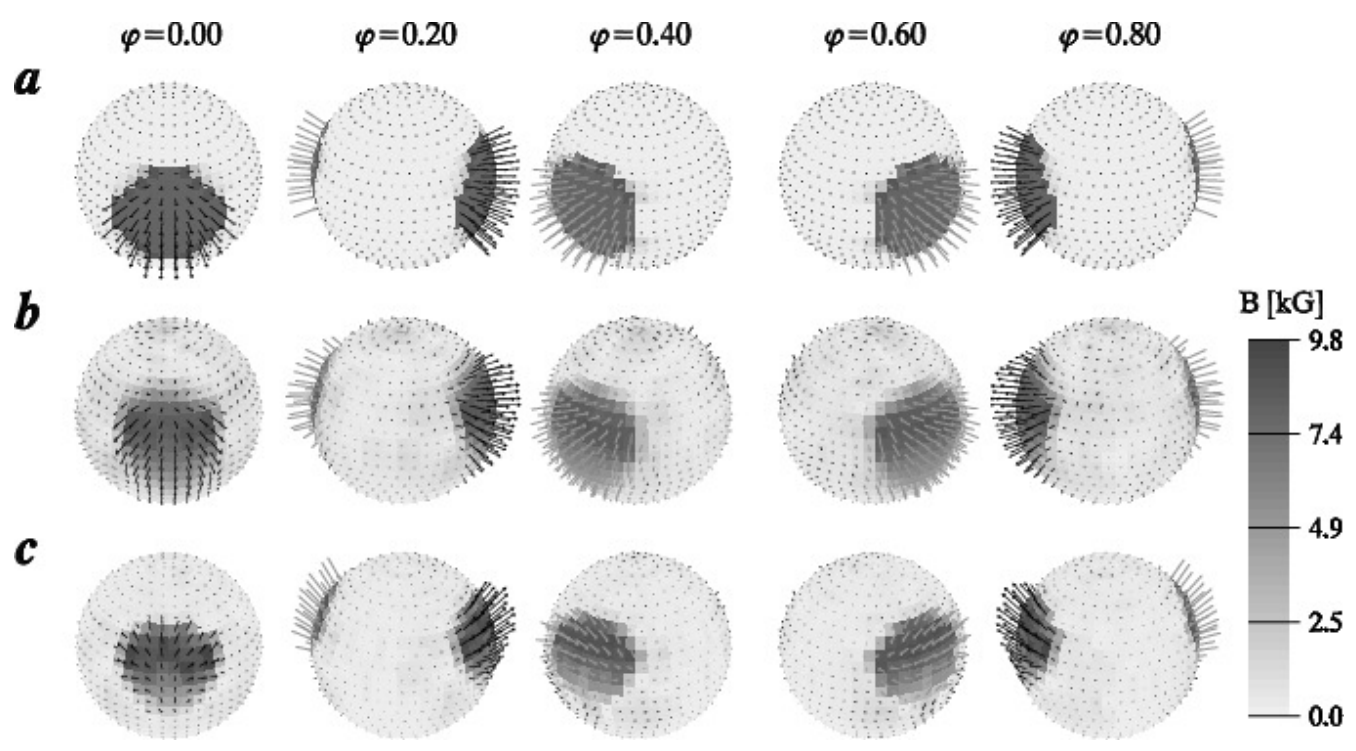

Figure 6: (a) Surface image consisting of two magnetic spots with $8 \mathrm{kG}$ radial field of opposite polarity, and (b) reconstructions involving all four Stokes parameters and (c) involving only Stokes I and V (from Kochukhov and Piskunov, 2002, reprinted with permission (C) ESO).

Two fundamental issues for Doppler Imaging techniques are that DI assumes the field not to be evolving, and that temperatures of magnetic regions are not generally known. The assumption of non-evolving fields is questionable given the high level of activity and rate of flaring of these stars, but we have only little information on characteristic timescales and evolution patters. Also, temperatures of stellar active regions are poorly known in stars other than the Sun, but regions of higher (lower) temperature add more (less) flux to the observed spectra than the quiet stellar photosphere.

The approaches to construct Doppler Images can be very different. It has been shown that relatively simple magnetic geometries can be reconstructed using all four Stokes parameters simultaneously and calculating magnetic radiative transfer. An example from Piskunov and Kochukhov (2002) is shown in Figure 6, another one from Donati (2001) is reproduced in Figure 7, and a third example from Donati and Brown (1997) is shown in Figure 8. There is an extensive literature on the applicability of ZDI that goes far beyond the scope of this review. For detailed information, the reader is referred to Donati and Landstreet (2009), Kochukhov and Piskunov (2002), and Donati (2001) and references therein. As a few examples, Figure 6 shows a reconstruction of a star with two magnetic spots (Kochukhov and Piskunov, 2002), Figure 7 show reconstructions of a largescale dipolar configurations (Donati, 2001) using different assumptions on the field structure, and Figure 8 shows a configuration with two relatively large spots (Donati and Brown, 1997).

In cool stars, no Zeeman Doppler Image from all four Stokes parameters exists today, but may become achievable with high-resolution spectro-polarimeters like PEPSI (Strassmeier et al., 2004). Because the signal in Stokes I is extremely weak at visual wavelengths and for magnetic fields much weaker than several kG (as used for example in Figure 6), even using only Stokes I and V together is usually not an option in cool stars (see also next section). Effects of using Stokes I and V, or Stokes V alone are shown in the examples in Figure 6 and 8. Neglecting Stokes Q and U leads to an underestimate of the area covered by the magnetic spots at low latitudes and to strong 


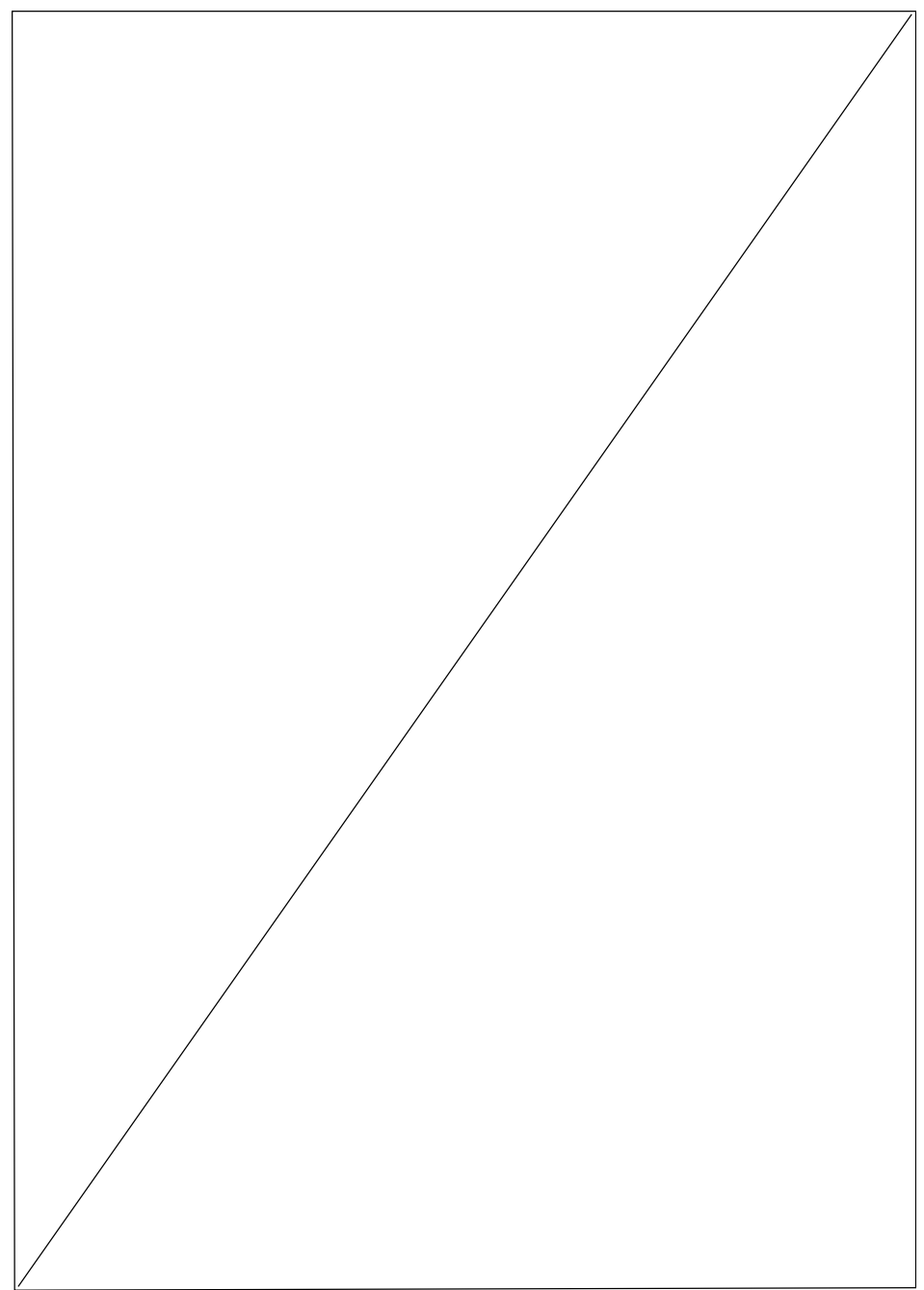

Figure 7: Removed figure post-publication due to copyright restrictions. Springer did not grant permission to reuse material "in a work to be published on an Open Access Website". Field reconstructions shown in a flattened polar projection with parallels drawn as concentric circles every $30^{\circ}$ down to a latitude of $-30^{\circ}$. Bold circle and central dot denote equator and visible pole, respectivey. Black and white code field intensities of $1000 \mathrm{G}$ and $-1000 \mathrm{G}$. Reconstructions of a synthetic dipole field (left panel) are shown assuming unconstrained field structure (center panel) and linear combination of force-free fields (right panel) (from Donati, 2001). 


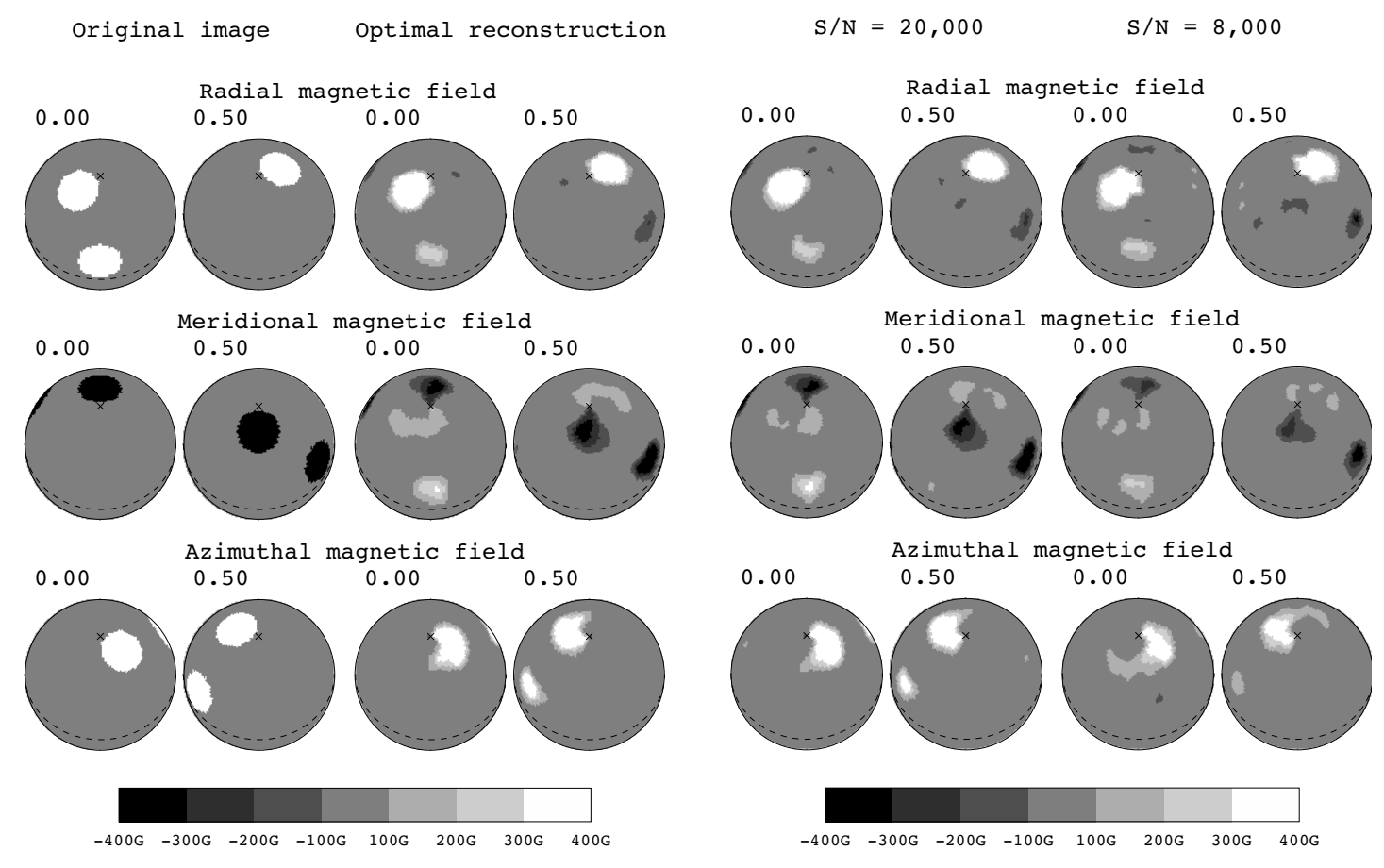

Figure 8: Six spot star simulations for a star observed under an inclination angle of $i=30^{\circ}$. The data set includes Stokes V profiles at 10 evenly spaced phases. The original images is shown in the left two columns. The next two columns show the optimal reconstruction followed by reconstructions with noise levels increased to $5 \times 10^{-5}(\mathrm{~S} / \mathrm{N}=20000)$ and $1.25 \times 10^{-4}(\mathrm{~S} / \mathrm{N}=8000)$ (from Donati and Brown, 1997, reprinted with permission (C) ESO).

crosstalk from the radial to the meridional field map while no crosstalk appears from the radial to the azimuthal maps (Kochukhov and Piskunov, 2002). Donati and Brown (1997), using examples with two large spots, show that imaging in Stokes V suffers essentially from crosstalk between low-latitude radial and meridional field features at low inclinations, but otherwise reasonably well recovers the input field structure. They also demonstrate how reconstructions deteriorate when data quality is lower (Figure 8). Another example addressing the crosstalk issue is given by Donati (2001) using examples of a large magnetic spot and dipolar magnetic field configurations.

Obviously, ZDI is a powerful method that can be used to recover useful information on stellar magnetic field configurations. While it is undisputable that pure large-scale fields are more easily observable than small-scale field components, and that crucial information about the large-scale surface magnetic field can be recovered, it is not entirely clear what part of a more complex field geometry is reconstructed under realistic conditions in low-mass stars (including cool spots and hot emission regions, small spot groups, and temporal evolution). A very practical limitation for the Doppler Imaging technique in cool stars is that extremely high signal-to-noise ratios are required in polarized light in order to measure the subtle signatures of net polarization. Simply integrating over long times in order to collect enough photons is not applicable because individual exposures for Doppler Imaging must be kept short enough so that adequate spatial resolution can be achieved. One way out is to use bigger telescopes, another is to cleverly co-add the information contained in the many spectral lines that all contain similar information from the star; this can be done with a technique called Least Squares Deconvolution. 


\subsubsection{Least Squares Deconvolution}

The basic idea of Doppler Imaging is to translate line profile variations into a map of the stellar surface. The information of the surface itself is contained in every spectral line, but each line is sampled with relatively high noise in the spectroscopic data. If one assumes that line formation is similar in all lines, the full spectrum can be described as a convolution between a broadening function characteristic of the stellar surface at a given rotational velocity, and the spectrum of the star as it would look if the star was not rotating. Least Squares Deconvolution (LSD, developed by Semel, 1989 and Donati et al., 1997) is the inverse process: assuming a non-broadened intrinsic spectrum of the star, one searches for the broadening function that must be convolved with this intrinsic function so that the result of the convolution provides the best match to the observed data. Donati et al. (1997) treat the observed spectrum as the convolution of the broadening function with a set of weighted "delta" functions located at the wavelengths taken from a spectral line list. Reiners and Schmitt (2003b) used a similar approach but iteratively optimizing the weights of individual lines so that the fit to the spectrum is improved.

In its simplest incarnation, LSD can provide the broadening function that is inherent in all spectral lines, and using many lines can boost the signal-to-noise ratio of the derived broadening function with respect to individual lines. Furthermore, line blending can be treated very effectively. LSD can provide an accurate measure of the broadening profile inherent to all spectral lines if one makes the assumption that the broadened template spectrum captures all differences between the lines used (e.g. Reiners and Schmitt, 2003a). This implies that lines are not allowed to follow different broadening patterns or line formation processes (Sennhauser and Berdyugina, 2010). As a consequence, lines with different Landé factors following different broadening patters cannot be used to derive a broadening profile that can be interpreted as the broadening profile inherent in each line. If the broadening patterns of individual spectral lines differ, however, LSD can still be used to determine an average broadening function from many lines. As an approximation for Zeeman broadening, average Landé $g$ values are sometimes assumed to derive an average Zeeman broadening profile in Stokes I (e.g., Morin et al., 2008). The interpretability of these signatures is limited (Sennhauser and Berdyugina, 2010) but can still allow a useful mapping of the stellar surface.

For polarized light, Donati et al. (1997) show an elegant way how LSD can be used to extract mean broadening profiles from circular polarization in Stokes V data, and Wade et al. (2000) extend this formalism to linear polarization. A crucial step is to apply the so-called weak-field approximation (see Unno, 1956; Stenflo, 1994): if Zeeman splitting is much smaller than the Doppler width of spectral lines, the following equations hold for every line $i$ :

$$
\begin{aligned}
& V(v) \propto g_{i} B \frac{\partial I(v)}{\partial v}, \\
& Q(v) \propto g_{i}^{2} B^{2} \frac{\partial^{2} I(v)}{\partial v^{2}},
\end{aligned}
$$

with $V$ and $Q$ the Stokes parameters, $g_{i}$ the Landé factor for line $i, B$ the magnetic field, and $v$ the Doppler velocity. Thus, under the weak-field assumption, polarized spectra can be written as a convolution between an average line profile $\left(\frac{\partial I(v)}{\partial v}\right.$ or $\left.\frac{\partial^{2} I(v)}{\partial v^{2}}\right)$ and a line list in which each line is weighted by its Landé factor. The amplitude of the deconvolved broadening function in is proportional to $B$ and $B^{2}$ in $V$ and $Q$, respectively. In the weak-field approximation (together with the weak-line approximation; Sennhauser and Berdyugina, 2010), all line profiles have identical shape and only differ in intensity, which allows the use of a linear multi-line approach like LSD, which makes interpretation of the derived profile relatively straightforward. If fields are strong enough so that Stokes V splitting patterns significantly differ in shape between different lines, or if several lines are saturated, the meaning of the derived function becomes less obvious. Several other methods that overcome these limitations like Principal Component Analysis (PCA; Martínez González

Living Reviews in Solar Physics

http://www. livingreviews.org/lrsp-2012-1 
et al., 2008) or Zeeman Component Decomposition (ZCD; Sennhauser and Berdyugina, 2010) were developed during the last years.

As was mentioned several times already, polarization signals from integrated observations of cool stars are so small that usually they cannot be detected in individual spectral lines with current instrumentation. If the weak-field approximation is used, it is difficult to assess how the reconstruction of magnetic fields is affected, in particular together with ZDI. Donati and Brown (1997) point out that the weak field approximation is in principle no longer valid for field strengths above $1.2 \mathrm{kG}$, but the authors claim that in special cases the weak field approximation can adequately describe Stokes V profiles up to $5 \mathrm{kG}$ (see also Donati and Collier Cameron, 1997). In summary, it appears not obvious that algorithms applying the weak field approximation are sensitive to (and can correctly interpret) the signatures of fields much larger than $1 \mathrm{kG}$. A potential consequence could be that they are not only insensitive to average fields above kG-strength, but would also systematically miss spatially small magnetic components with fields of this strength, as for example large spots similar to the largest sunspots.

\subsection{Broad band polarization}

Obtaining high resolution spectra of cool stars, in particular of very faint $\mathrm{M}$ stars and cooler objects, is challenging because the required signal-to-noise ratios are difficult to reach. It is, therefore, very desirable to develop a method to measure magnetic field properties from low-resolution spectroscopy or even photometry. Leroy (1962) proposed that broad band linear polarization can be caused by differences between saturation of $\pi$ and $\sigma$ components (Figure 5). Based on the observation of linear polarization in different filters by Koch and Pfeiffer (1976), Mullan and Bell (1976) present evidence for a magnetic field of $10 \mathrm{kG}$ strength on the bright spotted dwarf BY Dra. This scenario, however, was ruled out by several later measurements. Huovelin and Saar (1991) and Saar and Huovelin (1993) modeled broad band linear polarization in cool stars including polarization from scatter in the stellar atmosphere. They show that broad band polarization probably dominates over Rayleigh and Thomson scattering. Measurements of linear polarization in cool stars were reported, e.g., by Tinbergen and Zwaan (1981) and Alekseev (2003), but the correspondence to magnetic regions is not entirely clear. Alekseev (2003) show linear polarization depending on wavelength with higher degrees of polarization at short wavelengths. This dependence is expected if the signal comes from the magnetic surface of the star, but in some cases the detected polarization strongly exceeds the maximum level expected. Thus, a supplementary source of polarization is suggested, which is proposed to be most likely the remnant of a circumstellar disk. If such a disk is required, however, polarization due to magnetism and polarization from the disk are difficult to disentangle. Other potential sources of broadband linear polarization include light source anisotropy (Al-Malki et al., 1999) and stellar flares (Saar et al., 1994b).

Bagnulo et al. (2002) demonstrated a method they used to successfully measure magnetic fields in hot stars from low spectral resolution. Assuming that the weak-field approximation holds, they plot circular polarization $V$ against the derivative of intensity, $d I / d \lambda$ (see Equation (5)). If the weak-field approximation holds and the intensity derivative is proportional to Stokes $\mathrm{V}$, the longitudinal magnetic field can be determined from the slope of their relation (Figure 9). The method is fairly straightforward in hot stars with well-separated hydrogen lines that all have very similar Landé factors. The method has not yet been applied successfully to cool stars (understood here as stars of spectral type $\mathrm{F}$ and later) and it is not clear whether it would work given the large number of blended lines with very different Landé factors. Kolenberg and Bagnulo (2009) applied the method to RR Lyr stars that are technically similar to cool stars (regarding their outer convection zones) but have spectra very different to later spectral types. Nevertheless, this may be a promising method to determine longitudinal net field strengths in cool stars that are not observable at very high spectral resolution. 

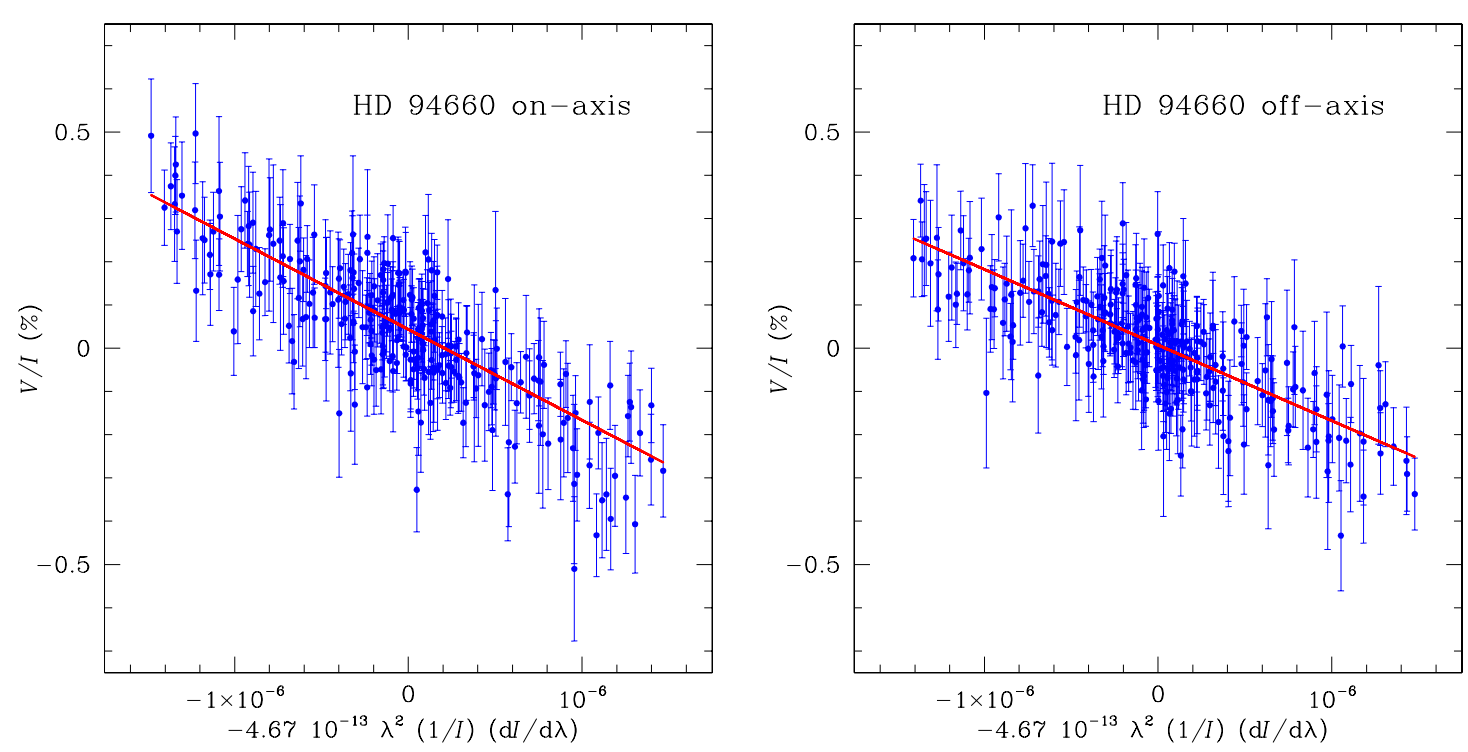

Figure 9: Stokes V plotted vs. the derivative of Stokes I (weak field approximation). The linear relation shows that the star has a magnetic field, and from the slope of the relation magnetic field strengths on the order of $2 \mathrm{kG}$ are derived for the two configurations (reprinted with permission from Bagnulo et al., 2002, (C) ESO).

\subsection{Indirect diagnostics}

We know from the Sun that magnetic regions lead to enhanced emission both in the solar chromosphere and in the corona. Chromospheric and coronal emission can be observed in tracers like Ca II emission in $\mathrm{H} \& \mathrm{~K}$ lines or the $\mathrm{Ca}$ triplet, in $\mathrm{H} \alpha$, in $\mathrm{UV}, \mathrm{X}$-ray, or radio emission. If we assume that other stars obey the same relations between magnetic fields and emission processes, we can determine their magnetic fields from observations of these tracers. For most of the indirect tracers, the determination of magnetic field requires: 1) that magnetic fields and their configuration in other stars are not too different from the solar field; and 2) that we correctly identify the mechanism coupling magnetic fields to observable emission. In this review, I will not give a detailed discussion of the results from indirect diagnostics, but rather introduce the general ideas and refer to the original literature.

\section{Intensity contrast}

Figure 1 gives a clear example of the correspondence between surface brightness and magnetic flux density on the Sun. Relations between these two values were provided, e.g., by Ortiz et al. (2002). They determine contrasts of active region faculae and the network as a function of heliocentric angle and magnetogram signal. Although this information is not available in spatially unresolved observations of other stars, it can be very helpful for the analysis of stellar variability from high quality photometric data, e.g., from the CoRoT or Kepler satellites.

\section{Chromospheric emission}

The correspondence between chromospheric CaII K emission and magnetic fields for the solar surface was investigated by Schrijver et al. (1989). Figure 10 shows that a close relation exists 
between the field strength and Ca II emission in solar surface observations:

$$
\frac{I_{c}-0.13}{I_{W}}=0.008\langle f B\rangle^{0.6}
$$

with $I_{C}$ the core intensity and $I_{W}$ the intensity in the wings (see Schrijver et al., 1989). Schrijver (1990) found a relation between C IV and magnetic flux density of the form

$$
F_{\mathrm{C} \text { iv }} \propto\langle f B\rangle^{0.7}
$$

A similar correspondence was also observed in M stars but using H $\alpha$ (Reiners and Basri, 2007, 2010). Disk-integrated measurements of stellar chromospheric activity can therefore trace changes in surface activity induced by, e.g., rotation or magnetic cycles (see, e.g., Baliunas et al., 1995; Hall, 2008).

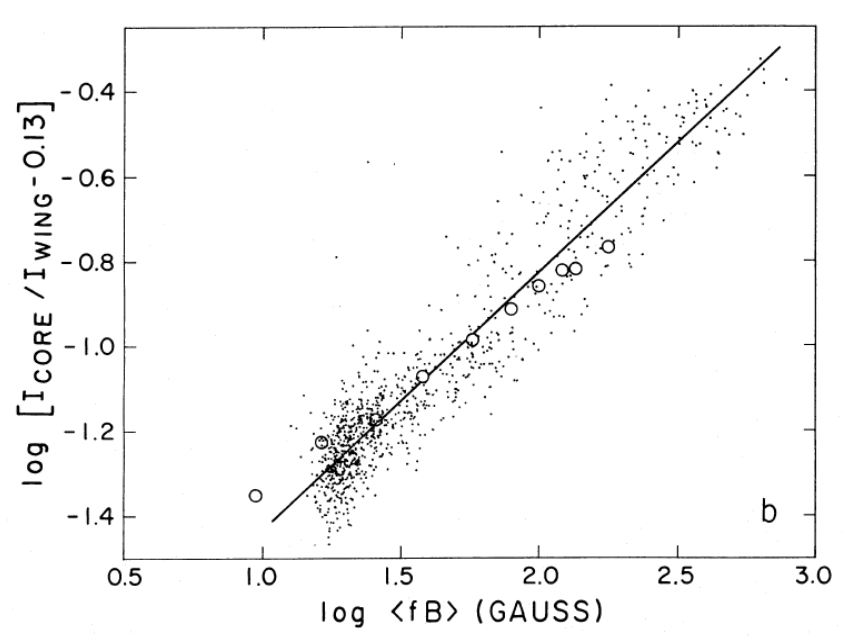

Figure 10: Ca II K core wing intensity ratio vs. absolute value of the magnetic flux density from resolved solar surface observations after degradation of the resolution to 14.4" × 14.4" (from Schrijver et al., 1989, reproduced by permission of the AAS).

\section{X-ray emission}

X-ray observations are available for the Sun and many stars. A close relation between magnetic flux and X-ray spectral radiance was shown by Pevtsov et al. (2003) (see also Güdel, 2004). The relation holds for solar quiet regions, active regions, and disk-integrated measurements of very active stars and covers more than ten orders of magnitude in both parameters (see Figure 11). The relation is approximated by

$$
L_{X} \propto \Phi^{1.15}
$$

with $L_{X}$ the X-ray spectral radiance and $\Phi$ the magnetic flux. The relation is similar to the one found by Saar (2001) for cool stars,

$$
F_{X} \propto \Phi^{0.95}
$$




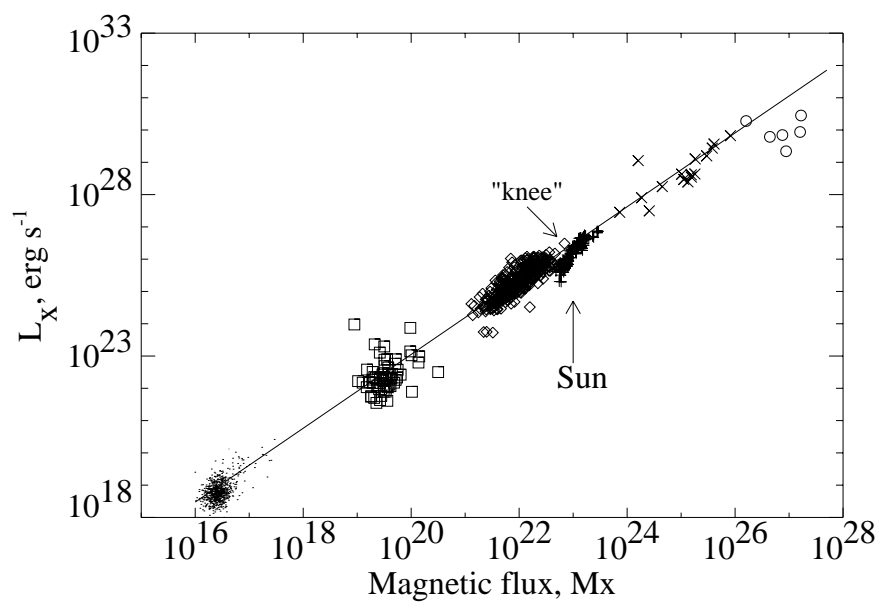

Figure 11: X-ray spectral radiance vs. total unsigned magnetic flux for solar and stellar observations. Dots: Quiet Sun. Squares: X-ray bright points. Diamonds: Solar active regions. Pluses: Solar disk averages. Crosses: G, K, and M dwarfs. Circles: T Tauri stars. Solid line: Power-law approximation $L_{X} \propto \Phi^{1.15}$ (from Pevtsov et al., 2003, reproduced by permission of the AAS).

\section{Radio emission}

Emission of radiation at radio wavelengths is indicative of ionized atmospheres that in many cases are related to stellar magnetic activity. Radio emission can be generated by different processes leading to characteristic signatures of radio emission; for an overview see Güdel (2002). The close correlation between radio and X-ray emission (the Güdel-Benz relation; Benz and Güdel, 1994) shows that radio and X-ray emission are generated by the same or at least correlated processes. The relation holds for quiescent and active emission of the Sun and a wide variety of stars. In very low-mass stars or brown dwarfs, however, Berger et al. (2005) showed that this relation is violated with objects that are overluminous at radio wavelengths.

Depending on the emission process, or on the question whether the emitting electrons are relativistic or not, radio emission has characteristic properties that can allow the determination of magnetic fields (see Güdel, 2002). The gyrofrequency, or cyclotron frequency, in a magnetic field is

$$
\nu_{c}=\frac{e B}{2 \pi m_{e} c} \approx 2.8 \times 10^{6} B,
$$

with the magnetic field strength $B$ in Gauss and $\nu_{c}$ in Hz. Gyrosynchrotron emission from a powerlaw electron distribution is proportional to $\gamma^{-\delta}$ (with $\gamma$ the Lorentz factor) and shows polarization characteristic for the magnetic field. For a chosen angle between the line of sight and the magnetic field, $\theta=\pi / 3$, the polarization $p$ can be written (Dulk, 1985)

$$
p \approx 10^{3.35+0.035 \delta}(\nu / B)^{-0.51} .
$$

In principle, this equation can be used to determine the magnetic field strength from the fractional polarization of radio emission, but it rests on several assumptions and is very sensitive to the geometry of the emitting regions, which is not known in spatially unresolved stars.

Coherent emission, in particular electron cyclotron maser emission, can be a reliable tracer of the magnetic field strength because it is emitted mostly at the fundamental and the second harmonic of $\nu_{c}$. Detection of radio emission at a given frequency indicates the presence of magnetic field corresponding to that frequency, for example the detection of $8.5 \mathrm{GHz}$ radio emission indicates a field of strength $B \geq 3 \mathrm{kG}$ (see, e.g., Hallinan et al., 2008). 


\title{
3 Magnetic Field Measurements in Cool Stars
}

\subsection{Average magnetic fields from integrated light}

\author{
As more physical effects were included in the modeling, \\ more sources of line broadening were treated and the mag- \\ netic parameters decreased. \\ But one must ask: will $f B \rightarrow 0$ eventually? \\ Saar (1996b)
}

The history of magnetic field measurements in cool and, in particular, in sun-like stars, is not easily followed. The fundamental paradigm of magnetic fields leading to chromospheric and coronal emission, as observed on the Sun, has motivated clear expectations on the presence and properties of magnetic fields. The relation between rotation and activity, hence presumably also between rotation and magnetic flux, and the difficulty to detect Zeeman signatures in rotationally broadened spectral lines causes great practical difficulty, especially in sun-like stars. In low-mass (M-type) and pre-main sequence stars, the relation between activity and rotation is presumably more observerfriendly, facilitating the detectability of Zeeman broadening. I will, therefore, distinguish between magnetic field observations in sun-like stars, low-mass stars, and pre-main sequence stars.

\subsubsection{Sun-like stars}

The general difficulties detecting the subtle effects of Zeeman broadening in a spectral line from the spatially unresolved stellar disk were discussed in Section 2.1. A promising way to overcome the problem of degeneracies between Zeeman broadening and other broadening agents is to compare spectral lines with different Zeeman sensitivities in the same spectrum. An enhanced width (or equivalent width) of the magnetically sensitive lines often is good indication for the presence of a magnetic field. This strategy was successfully applied to Ap stars with fields of $1 \mathrm{kG}$-strength by Preston (1971). Vogt (1980) used a multichannel photoelectric Zeeman analyzer mainly to measure polarization in sun-like stars, but also presents comparison between the widths (FWHM) of magnetically sensitive lines at $6173 \AA$ and two nearby, magnetically less sensitive lines. Four stars were analyzed with this method finding no evidence for magnetic fields. Vogt (1980) concludes that this rules out the presence of non-coherent longitudinal fields in excess of $1000-1500 \mathrm{G}$ and covering the entire surface, which is similar to $B f \leq 1500 \mathrm{G}$.

Robinson Jr (1980) introduced a new method based on the comparison between magnetically sensitive and insensitive lines. Realizing that the increase in line width for fields less than several $\mathrm{kG}$ is very small at optical wavelengths, he suggested to employ a Fourier transform technique to easily separate the broadening effects due to magnetism from other broadening effects. The underlying principle is the very same as if one is comparing line shapes or line widths directly in the wavelength regime (instead of Fourier regime). However, the Fourier transform technique is able to cleanly separate the different broadening effects at least in principle, and thus could ideally separate magnetic broadening from other effects. The main limitation of Zeeman broadening measurements at optical wavelengths, however, cannot be overcome by this method: it is still necessary to precisely measure a magnetically non-broadened line in order to use it as a template for the (potentially stronger) broadening observed in a magnetically sensitive line. Both lines must be of very similar nature in terms of formation height and temperature response. It is, therefore, not surprising that the limitations discussed by Robinson Jr (1980) are essentially identical to the limitations arising when line widths are compared directly. Consequently, the Fourier technique was not applied to a great many spectra, but the paper became a benchmark for line comparison 
techniques in general because it thoroughly discusses the requirements and limitations of this technique.

What followed was a series of attempts trying to measure magnetic fields in more or less active sun-like stars. Driven by detections of chromospheric and coronal activity, active stars with relatively low rotational broadening $(v \sin i)$ were observed in order to search for the effects of Zeeman broadening. Highest obtainable data quality at this time was typically on the order of $R \sim 50000-$ 70000 and SNR $\sim 100-200$. A remarkable conclusion from the magnetic field observations taken during this time was pointed out by Gray (1985). Investigating the reports on magnetic field measurements, he finds that for G- and K-dwarfs, the product between the magnetic field strength $B$, and the areal coverage factor $f$, i.e., the average magnetic field strength $B f$, "is a constant independent of physical parameters such as spectral type and rotational velocity". Realizing that this is rather unlikely, he concludes that "either we have systematic misconceptions involved in our Zeeman-broadening analysis or else we have before us a remarkable magnetic conservation condition". The value of this "magnetic constant" is roughly $B f=500$ G. According to Equation (4), this means an extra-broadening of $700 \mathrm{~m} \mathrm{~s}^{-1}$ for a magnetically sensitive line $(g=2.5)$ over an insensitive line $(g=1.0)$ at red optical wavelengths $(670 \mathrm{~nm})$; this is typically between $10 \%$ and $20 \%$ of a resolution element.

This example demonstrates that searching for the subtle effects of a several hundred Gauss magnetic field is close to the theoretical detectability of the Zeeman effect, and that it is extremely difficult to judge whether differences between lines of different magnetic sensitivities are really due to magnetism. Consequently, the Zeeman analysis methods were criticized by many authors (see e.g., Saar, 1988) centering on two flaws: 1) incomplete treatment of radiative transfer, and 2) lack of correction for line blends. Saar (1988) presents a set of improved methods for the analysis of magnetic fields in cool stars. Main ingredients are radiative transfer effects, treatment of exact Zeeman patterns, and improved correction for line blends. Following up on this improvement, Basri et al. (1990) went one step further introducing a two-component analysis by applying their more detailed line-transfer analysis to the (more realistic) situation in which the magnetic component of the stellar atmosphere is not identical to the non-magnetic component. The authors also point out that the derived magnetic flux still could be in error by a factor of 2 because atmospheres from one-dimensional calculations are used for a multi-component analysis (neglecting gradients and differences in atmospheric structure); misestimates of abundance, turbulence, and subsequently magnetic field can be quite severe. A detailed parameter study estimating the accuracy of magnetic field analysis methods in detailed radiative transfer calculations with embedded fluxtubes is given by Saar and Solanki (1992) and Saar et al. (1994a).

Obviously, a straightforward way to improve magnetic field measurements is to observe at longer wavelengths (see Equation (4)). Useful lines are found for example at $1.56 \mu \mathrm{m}$ (Fe I) and $2.22 \mu \mathrm{m}$ (Ti I), i.e., at wavelengths a factor of 3-4 longer than typical red/optical observations. First suitable instrumentation at such long wavelengths became available in the early-1990s. The first detailed analysis of a high-resolution infrared spectrum in a sun-like star (for earlier work on M stars, see Section 3.1.2) was performed by Valenti et al. (1995). These authors used a highresolution $(R=103000)$, high SNR $(100-200)$ spectrum (taken during several hours of exposure) to determine the magnetic field of $\epsilon$ Eri, and upper limits on the order of $100 \mathrm{G}$ in two other early K-dwarfs. $\epsilon$ Eri has been subject to magnetic field investigations many times earlier at optical wavelengths. Valenti et al. (1995) also show a compilation of reports on magnetic field measurements in this star published between 1984 and their work in 1995. Interestingly, average magnetic fields of $\epsilon$ Eri decreased over time starting at $\sim 800 \mathrm{G}$ in 1984 and reaching $130 \mathrm{G}$ in 1995. Possible interpretations of this result are that the field in $\epsilon$ Eri is variable, or that observations reporting lower field strengths (predominantly near-IR measurements) probe a different part of the stellar atmosphere. Valenti et al. (1995) discuss possible scenarios reaching the conclusion that probably optical investigations have overestimated the magnetic flux of $\epsilon$ Eri.

Living Reviews in Solar Physics

http://www. livingreviews . org/lrsp-2012-1 
A critical compilation of magnetic field measurements obtained between the paper of Robinson Jr (1980) and 1996 was attempted by Saar (1996b). The selection process leading to a condensed sample of "improved" field measurements was described as follows: "I have therefore compiled a carefully selected sample of magnetic measurements from analyses which treat radiative transfer effects and use disk-integration in their models. In addition, I (ruthlessly!) neglect results from low S/N IR data, measurements using Fe I $8468 \AA$ in $K$ dwarfs, Zeeman/magnetic Doppler imaging results, and curve-of-growth analyses" (for the reasons why some techniques were neglected, see Saar, 1996b). A similar, upgraded collection of Zeeman analyses carried out in the period 1996-2001 was given by Saar (2001).

For this review, I have tried in Table 1 to compile magnetic field measurements available for sunlike stars. Following Saar (1996b), I include only those measurements that rely on relatively high data-quality and analysis techniques. Since apparently not very many magnetic field measurements were reported in sun-like stars after 2001, Table 1 does not contain many results in addition to the compilations by Saar (1996b, 2001). However, in the light of the results reported by Valenti et al. (1995), I distinguish between work done at optical wavelengths and work done at infrared wavelengths, the former probably being more prone to overestimating the magnetic field.

A critical re-investigation of the detectability of magnetic fields in high-quality optical spectra was carried out by Anderson et al. (2010). The data material used for this work is of much higher quality than most magnetic field investigations before, and the data therefore allows a critical view on the published results and some of the limitations of the method. Anderson et al. (2010) used optical spectra around the Fe I line at $6173 \AA$ observed at a spectral resolving power of $R=220000$ and SNR $\sim 400$. The analysis is carried out for a one-component model with the same atmosphere for the magnetic and the non-magnetic parts of the stellar surface, and also for a two-component model employing different atmospheres for the two components. The results are reproduced in Figures 12 and 13. For the active G0 star 59 Vir, the authors find a magnetic field with $B f \approx 420 \mathrm{G}$ for the one-component case. For the two-component analysis, they cannot exclude a zero-field solution reporting an upper limit of $300 \mathrm{G}$. Figure 12 shows how subtle the differences between solutions with different magnetic field strengths are if all other relevant parameters are allowed to vary freely (there is currently no way to constrain these parameters at the level required). Figure 13 demonstrates the relation between magnetic field strength $B$ and filling factor $f$ in case of a one-component atmosphere (left panel). The two-component models shown in the center and right panels, however, can lift the $B f$ degeneracy but manage to reproduce the spectra even without the presence of a significant magnetic field. In other words, at optical wavelengths, the signal of temperature spots on the surface of a cool star can dominate the influence of the magnetic field through Zeeman broadening. Unfortunately, we have so far no clear empirical evidence for the relation between temperature and magnetic field strength on stellar surfaces other than on the Sun.

A look at Table 1 reveals that infrared measurements are only available in six sun-likes stars, all of them are of spectral type K. Two of the six data points are actually non-detections, and three were reported in conference summaries in which, unfortunately, no comprehensive presentation of the data and its analysis is given.

\subsubsection{M-type stars}

Low-mass stars of spectral type $\mathrm{M}$ have radii of approximately half a solar radius and less. If the stellar dynamo depends on the value of the Rossby number, $R o=P / \tau_{\text {conv }}$, the magnetic field strength expected in sun-like and low-mass stars is a function of rotational period and convective overturn time. Values for the convective overturn time are theoretically not well determined, but $\tau_{\text {conv }}$ is probably higher at lower masses (e.g., Kim and Demarque, 1996). Therefore, slower rotation is sufficient to produce larger fields in less massive stars. Furthermore, the smaller radii of less 

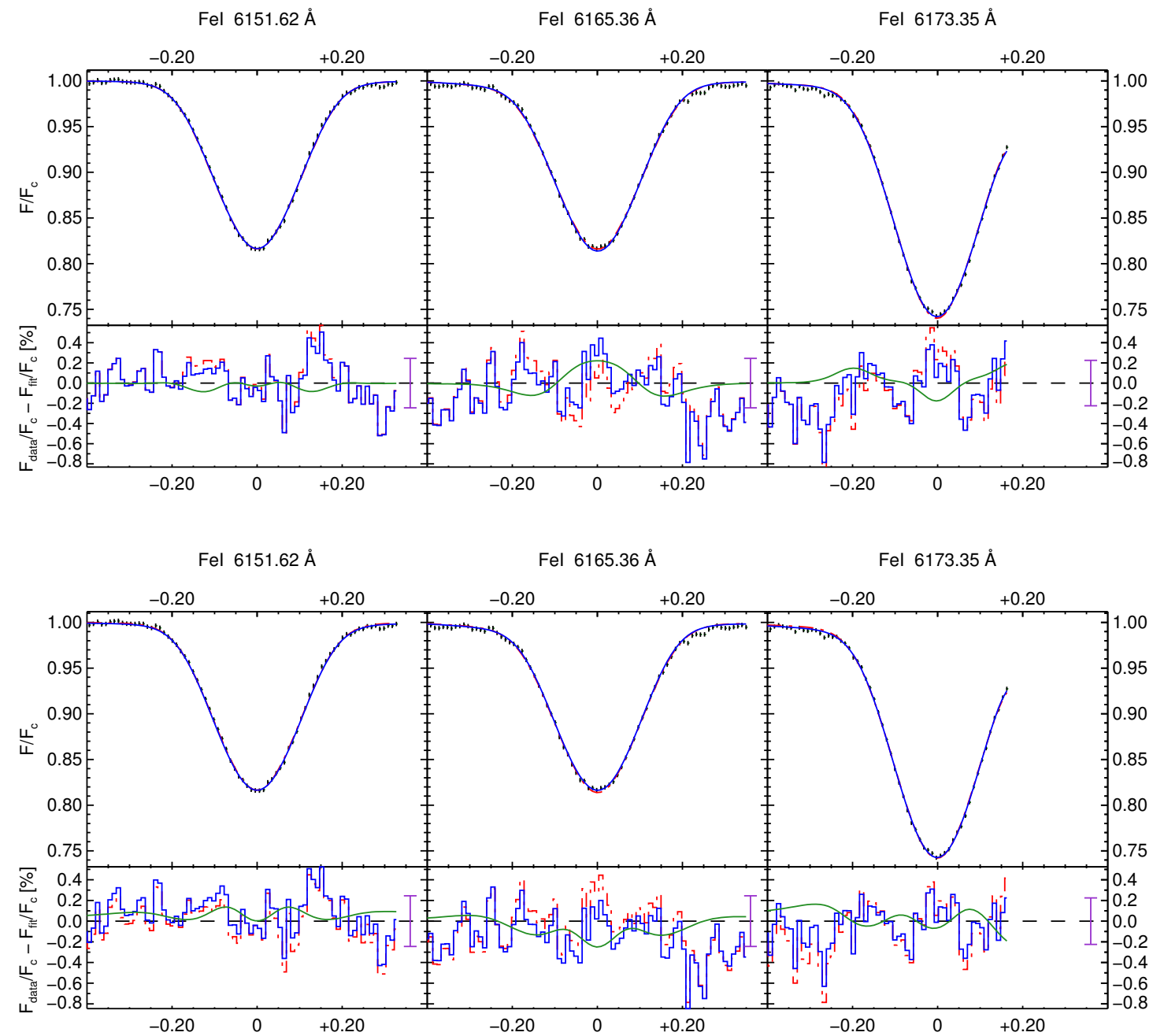

Figure 12: CES spectra of 59 Vir with uncertainties overplotted by best-fit solutions. Solid blue lines represent the overall best-fit solutions, dash-dotted red lines are other solutions shown for comparison. Residuals drawn below the fits visualize differences between measured and calculated line profiles, scaled by factor 100. The purple error bar to the right shows $\sigma_{i}$. Green lines indicate the difference between overall best-fit and the comparison model, i.e., the change in line shape due the presence of magnetic flux (other fit parameters vary freely). Top: Model with identical temperature for magnetic and non-magnetic regions; best-fit: $B f=500 \mathrm{G}$ (solid blue), comparison: $B f=0 \mathrm{G}$ (dash-dotted red). Bottom: Best fit for model with different temperatures for magnetic and non-magnetic regions; $B f=120 \mathrm{G}$ (blue solid), comparison: solution from upper panel ( $B f=500 \mathrm{G}$, same temperatures, red dashed line) (from Anderson et al., 2010, reprinted with permission (C) ESO). 

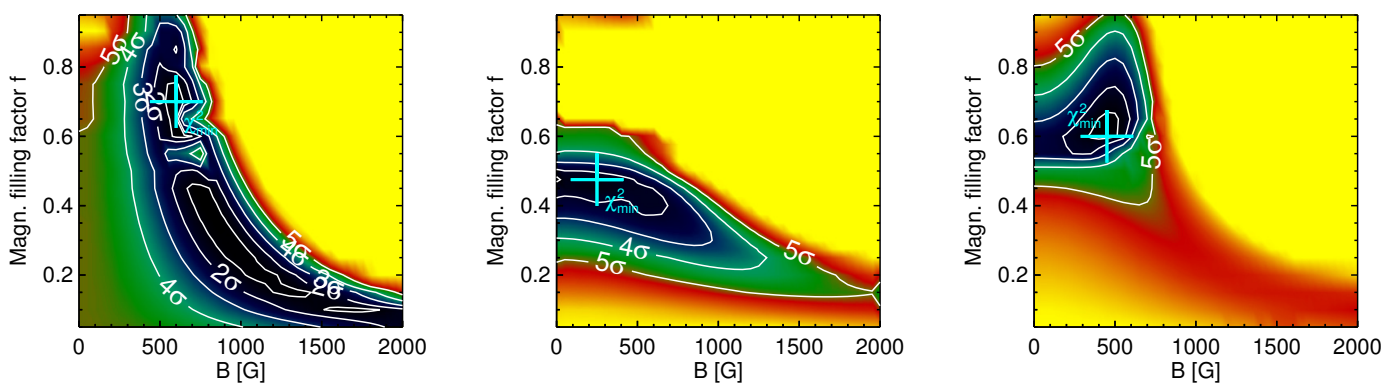

Figure 13: $\chi^{2}$-maps for 59 Vir solutions (see Figure 12). Left panel: Solution using the same atmospheres for magnetic and non-magnetic regions; Center panel: the same using cool magnetic regions; Right panel: the same for warm magnetic regions (from Anderson et al., 2010, reprinted with permission (C) ESO).

massive stars lead to lower surface velocities hence less rotational broadening at a given rotational period. Finally, less massive stars are also much cooler thus exhibiting less temperature broadening in their spectral lines. It is this combination of parameters that facilitates the detection of Zeeman splitting in M-type stars in comparison to more massive, sun-like stars; Zeeman broadening is more easily detected because of generally narrower line widths (see also Reiners, 2007).
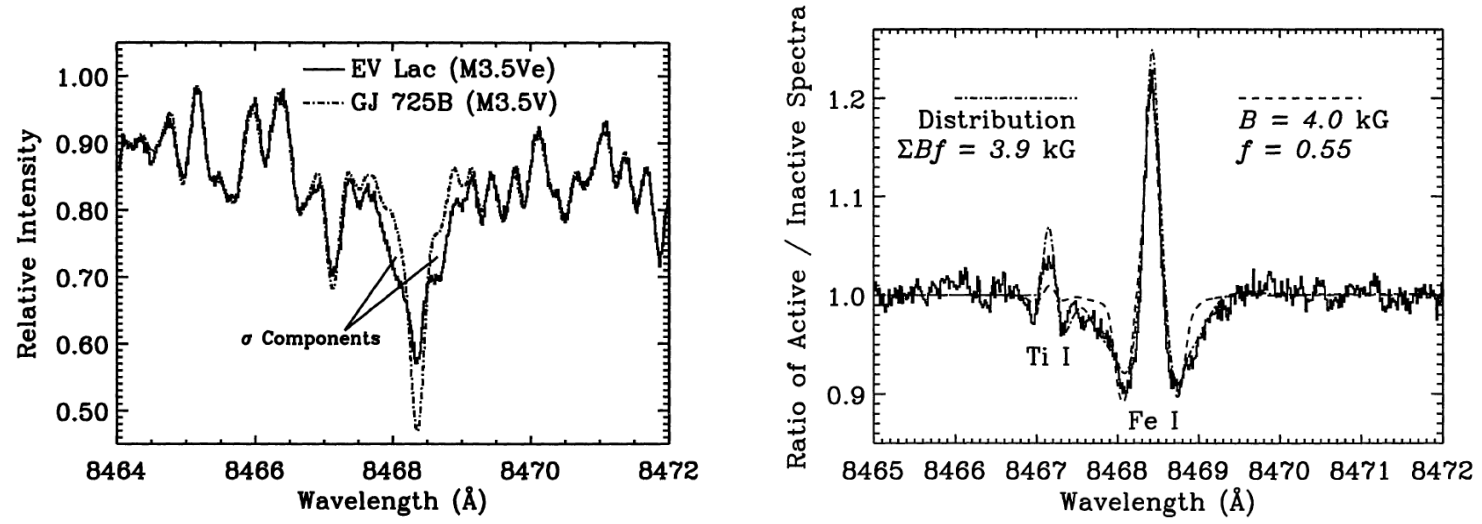

Figure 14: Magnetic field measurements in active $M$ dwarfs. Left: Spectra of the flare star EV Lac and the inactive star Gl 725B in the vicinity of the magnetically sensitive Fe I line at $8468.4 \AA$. Right: The spectrum of EV Lac divided by the inactive star Gl 725B (solid histogram). The dashed line shows a single field fit to the data (missing the line wings), the dashed-dot line show a fit allowing a distribution of magnetic fields (from Johns-Krull and Valenti, 2000).

The first detection of Zeeman splitting in an M-type star, and also the first detection of a photospheric magnetic field in cool stars at all, was presented by Saar and Linsky (1985). They observed the early-M flare star AD Leo using a Fourier transform spectrometer. After six hours of observation they had obtained a spectrum with $R=45000$ and SNR $\approx 25$ around the Ti I lines at $2.22 \mu \mathrm{m}$ from which they measured an average magnetic field strength of $B f=2800 \mathrm{G}$. Similar data taken with the same instrument was obtained in a few M-stars, and Saar (1994) presented a preliminary analysis of the three M-type stars AU Mic, AD Leo, and EV Lac. Another benchmark was the investigation of the Fe I line at $8468 \AA$ in seven early- to mid-M dwarfs by Johns-Krull and Valenti (1996). Substantial magnetic fields were detected in two stars of the sample, EV Lac and Gl 729. A refined analysis of the two stars and AD Leo and YZ Cmi was presented in Johns- 
Krull and Valenti (2000). The latter work assumed a distribution of magnetic fields on the stellar surface, which led to significantly higher average field values compared to Johns-Krull and Valenti (1996). The results from the $8468 \AA$ line were comparable to the values from the $2.22 \mu \mathrm{m}$ line within $10-20 \%$.

A serious problem for the detection of Zeeman splitting in atomic spectral lines of M-type stars is the appearance of molecular bands. For example, the Fe I line at $8468 \AA$ is embedded in a forest of $\mathrm{TiO}$ molecular absorption lines, which makes the modeling of Zeeman splitting in this line a delicate task. To overcome this problem, and the notorious difficulty to model TiO absorption (see Valenti et al., 1998), Johns-Krull and Valenti (1996) modeled the ratio of the flux between an active and an inactive star. Hopefully, our understanding of very cool atmospheres, molecular chemistry, and molecular line formation will in the future allow a detailed modeling of Zeeman splitting in the spectra of M dwarfs (see also Kochukhov et al., 2009; Önehag et al., 2011; Shulyak et al., 2011).

At optical wavelengths, the main opacity contributors in $\mathrm{M}$ dwarfs are molecular bands from $\mathrm{TiO}$ and VO. Analysis of Zeeman broadening in these bands, however, is difficult not only because of problems getting the line formation right, but also because the lines are not individually resolved. Nevertheless, for the detection of M star magnetic fields, it would be favorable to utilize molecular absorption bands. A molecular band that appears to be extremely useful for the analysis of Mstar magnetic fields (and other purpose) is the near-infrared band of molecular FeH. Its suitability for magnetic analysis was shown by Wallace et al. (1999), and it was proposed to be a useful diagnostic at low temperatures by Valenti et al. (2001). An observational problem of FeH is that its most suitable band is located at around $\lambda=1 \mu \mathrm{m}$, which is too red for most CCDs and too blue for most astronomically used infrared spectrographs. As a consequence, only very few high-resolution spectrographs can provide spectra at this wavelength, and efficiencies are typically ridiculously low. On the other hand, $\mathrm{M}$ dwarfs emit much of their flux at near-infrared wavelengths so that in comparison to optical measurements, the signal quality around $1 \mu \mathrm{m}$ is not much lower than around $700 \mathrm{~nm}$ if the spectra are obtained with an optical/near-IR echelle spectrograph like HIRES (Keck observatory) or UVES (ESO/VLT). Reiners and Basri (2006) developed a method to semi-empirically determine the magnetic fields of $\mathrm{M}$ dwarfs comparing $\mathrm{FeH}$ spectra of the targets to spectra of two template stars; one with no magnetic field and one with a known, strong magnetic field (Figure 15). This method requires a known magnetic star to calibrate the Zeeman splitting amplitude. The field strength of the target star is then estimated by interpolation between the template spectra.

The method of Reiners and Basri (2006) was first used in a sample of $24 \mathrm{M}$ stars between spectral types M0 and M9 (Reiners and Basri, 2007). As reference, the field measurement of EV Lac measured by Johns-Krull and Valenti (2000) was used. Thus, all magnetic field measurements are relative to this reference star $(\langle B\rangle=3.9 \mathrm{kG})$, and magnetic fields higher than this value cannot be quantified. Obviously, systematic uncertainties of the measurements are quite large, typically several hundred Gauss, and uncertainties probably grow towards very late spectral types where the template spectra are less suited as a reference. Unfortunately, Zeeman splitting of the FeH molecule is very complicated and could not entirely be described at this point (see Berdyugina and Solanki, 2002). Meanwhile, progress has been made using an empirical approach to understand FeH absorption and line formation (Wende et al., 2009, 2010), and to model Zeeman splitting in FeH lines (Afram et al., 2009; Shulyak et al., 2010). It was suggested that the fields determined semi-empirically may be overestimated by some $\sim 20 \%^{1}$ (Shulyak et al., 2010).

A (probably non-exhaustive) list of magnetic field measurements from Stokes I analysis in $\mathrm{M}$ dwarfs is given in Table 2, and I plot the distribution of field strength as a function of spectral type in Figure 16. The field strengths of young, early-M and field mid- and late-M dwarfs are

\footnotetext{
1 This could be due to an overestimate of the reference magnetic field measurement derived from the atomic line analysis.
}

Living Reviews in Solar Physics

http://www. livingreviews.org/lrsp-2012-1 


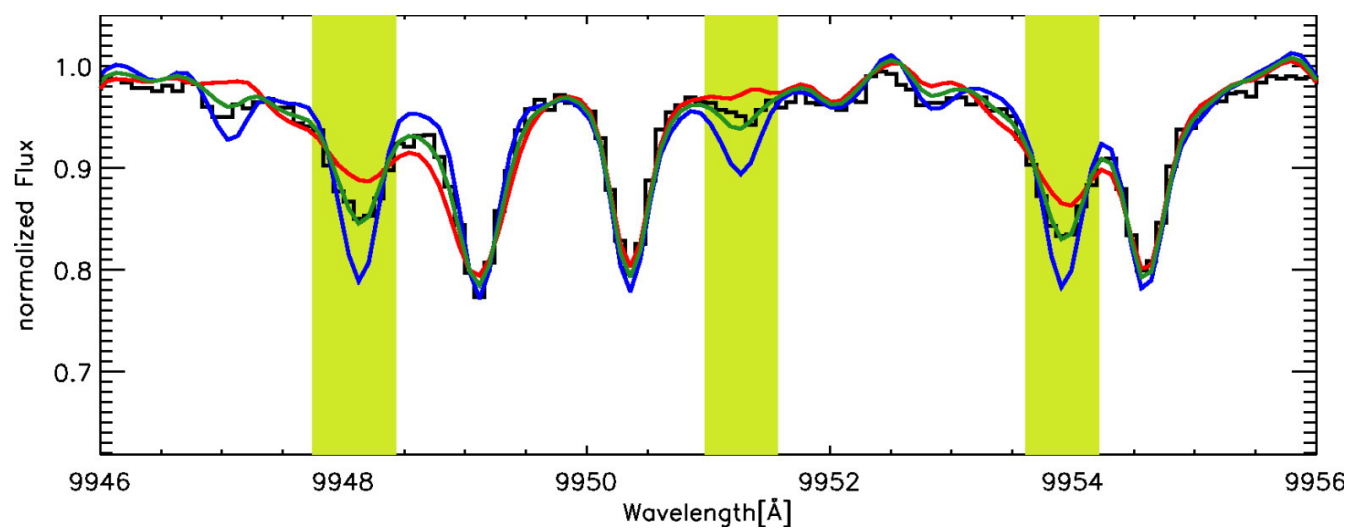

Figure 15: Magnetic field measurement using the empirical method of Reiners and Basri (2006). The black histogram shows the spectrum of Gl 729. The red and blue lines are scaled spectra of the active star EV Lac and the inactive star GJ 1002, respectively. The green line is an interpolation between the red $(B f=3.9 \mathrm{kG})$ and blue $(0 \mathrm{kG})$ lines yielding a field strength of $B f=2.2 \mathrm{kG}$ for Gl 729 (from Reiners and Basri, 2007, reproduced by permission of the AAS).

on the order of a few kG. This is the main results from Zeeman analysis and consistently found using different indicators (at least in mid-M dwarfs). Compared to the Sun, the average magnetic field hence is larger by two to three orders of magnitude, an observational result that must have severe implications for our understanding of low-mass stellar activity. It is not clear whether our picture of a star with spots more or less distributed over the stellar surface is actually valid in $\mathrm{M}$ dwarfs. If, for example, $50 \%$ of the surface of a star with a mean magnetic field of $4 \mathrm{kG}$ is covered with a "quiet" photosphere and low magnetic field, the other half of the star must have a field strength as large as $\sim 8 \mathrm{kG}$. The two components of the stellar surface on such a star probably have very different temperatures and properties, and the definition of effective temperature must be considerably different from the temperature of the "quiet" photosphere.

In early-M dwarfs (M3 and earlier), magnetic fields were found in young stars that are still rapidly rotating. Since old, early-M dwarfs in the field are generally slowly rotating and inactive there has been no search for magnetic fields in any large sample of them. Typical field values can be expected to be on the level of a few hundred Gauss and less, which is difficult to detect with Stokes I Zeeman measurements. Many mid- and late-M stars are rapidly rotating and fields of kG-strength are ubiquitously found among them.

\subsubsection{Pre-main sequence stars and young brown dwarfs}

Magnetic fields of pre-main sequence stars are of particular interest because accretion of circumstellar material onto the stellar surface is believed to be controlled by the stellar magnetic field (e.g., Bouvier et al., 2007). Evidence for accretion is observed in pre-main sequence stars of very different mass including young brown dwarfs. Field strengths predicted from several models of magnetospheric accretion are on the order of several $\mathrm{kG}$ for $\mathrm{T}$ Tauri stars, and a few hundred Gauss for young brown dwarfs (Johns-Krull et al., 1999b; Reiners et al., 2009b). On top of this, at young ages, magnetic fields may be generated by a dynamo like in older, sun-like stars (in contrast to fossil fields), but the dynamo would probably operate similar to the one in low-mass M-type dwarfs because pre-main sequence stars are still fully convective. On the other hand, at ages of a few Myr, primordial fields may still be present and not (yet) dissipated. Magnetic fields in pre-main sequence stars may therefore carry important information about the star- and planet-formation process. 


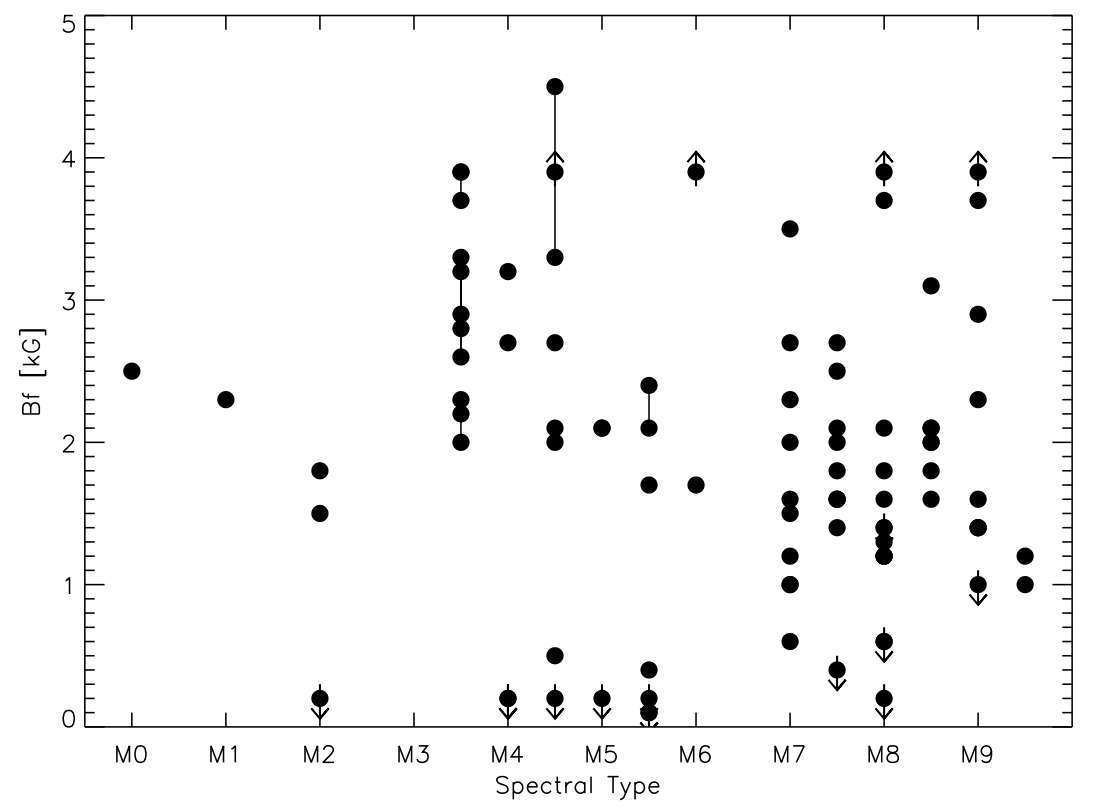

Figure 16: Measurements of $M$ dwarf average magnetic fields from integrated light measurements. Data are given in Table 2. Limits are indicated by arrows, and multiple measurements of the same star are connected with vertical lines.

First measurements of magnetic field strengths in $\mathrm{T}$ Tauri stars were attempted using the equivalent width method in (red) optical absorption lines by Basri et al. (1992), and Guenther et al. (1999) were following this strategy. Johns-Krull et al. (1999b) used infrared lines of Ti I at $2.22 \mu \mathrm{m}$ to determine the magnetic field in BP Tau. Obtaining information on stellar parameters and rotation from optical lines and magnetically insensitive CO lines, they were able to disentangle the significant Zeeman broadening from other broadening agents. Similar work on other T Tauri stars using infrared spectra was done by Johns-Krull et al., much of it is summarized in Johns-Krull (2007) where additional measurements of 14 T Tauri star magnetic fields are presented. Another set of 14 magnetic field measurements in very young $\mathrm{T}$ Tauri stars in the Orion nebula cluster are given in Yang and Johns-Krull (2011). We will return to the results from these substantial samples in Sections 5 and 7.3. A summary of magnetic field measurements in very young stars and brown dwarfs is given in Table 3, and are shown in Figure 17.

Using FeH measurements as laid out in Section 3.1.2, Reiners et al. (2009b) attempted to find evidence for kG-strength magnetic fields in young brown dwarfs. Young brown dwarfs can be expected to harbor substantial magnetic fields (Reiners and Christensen, 2010), and no fundamental difference is known to exist in the parameters that are believed to be relevant for magnetic flux generation between very-low mass stars and young brown dwarfs. However, in contrast to pre-main sequence stars, and in contrast to older brown dwarfs, none of the young brown dwarfs investigated by Reiners et al. (2009b) exhibited a field above the detection threshold that in all cases lay below the fields typically found among the other groups.

An important property of the four young brown dwarfs investigated for magnetic fields is that all of them show evidence for accretion and, therefore, harbor a circumstellar disk. Magnetic field strengths required for magnetospheric accretion in these objects are much lower than in more massive, young stars, hence there is currently no contradiction between the presence of accretion

Living Reviews in Solar Physics

http://www. livingreviews.org/lrsp-2012-1 


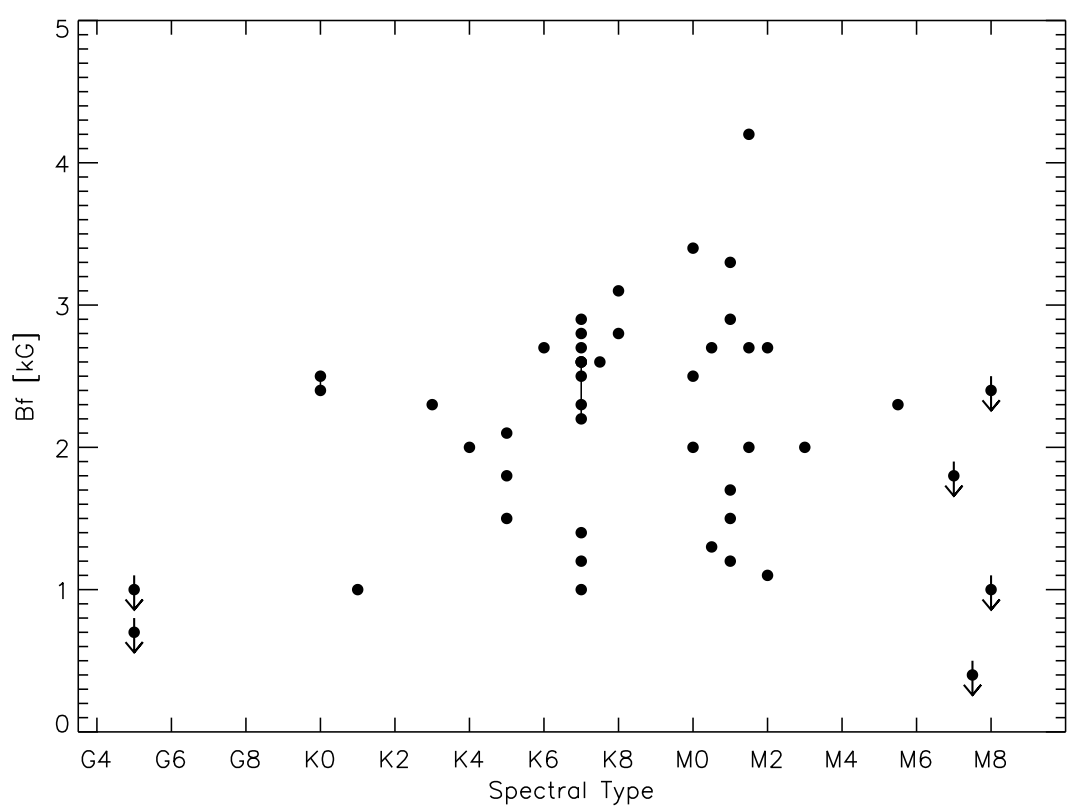

Figure 17: Measurements of pre-main sequence and young brown dwarf magnetic fields from integrated light measurements. Data are given in Table 3. Limits are indicated by arrows, and multiple measurements of the same star are connected with vertical lines.

and the lack of evidence for substantial fields. Observations of radio-emission, however, indicate that fields of a few $\mathrm{kG}$ strength are in fact present on some L-type field (old and non-accreting) brown dwarfs (Hallinan et al., 2008; Berger et al., 2009). Direct measurement of magnetism in non-accreting brown dwarfs, both young and old, are required to further investigate whether the average fields are really weaker in young brown dwarfs or in the presence of accretion.

It is an interesting question whether the non-detection of magnetic fields in brown dwarfs is due to the presence of accretion disks around the objects observed so far. If this is the case, there ought to be some mechanism for the disk to regulate the magnetic field of the central object, which is not easily understood. Alternatively, large difference in radius may be of importance in this context because the surface area of young brown dwarfs is about an order of magnitude larger than the surface of old brown dwarfs. If magnetic flux is approximately conserved during its evolution, the average magnetic field would be an order of magnitude lower in young, large brown dwarfs than in old, small, field brown dwarfs. We come back to this point in Section 7.3. 
Table 1: Average magnetic fields from Stokes I measurements in sun-like stars. Tables $1-7$ are an attempt to collect information available on magnetic fields in cool stars. They are certainly incomplete to some extent simply because the author has overlooked many sources. The reader is encouraged to send references to papers that are missing so far and new work that appears in this field.

\begin{tabular}{|c|c|c|c|}
\hline Star & SpType & $B f[\mathrm{kG}]$ & Reference \\
\hline \multicolumn{4}{|r|}{ IR data } \\
\hline$\sigma$ Dra & K0 & $\leq 0.10$ & Valenti et al. (1995) \\
\hline 40 Eri & K1 & $\leq 0.10$ & Valenti et al. (1995) \\
\hline$\epsilon$ Eri & K2 & 0.13 & Valenti et al. (1995) \\
\hline LQ Hya & $\mathrm{K} 2$ & 2.45 & Saar (1996b) \\
\hline$\xi$ Boo B & K4 & 0.46 & Saar (1994) \\
\hline Gl $171.2 \mathrm{~A}$ & K5 & 1.40 & Saar (1996b) \\
\hline \multicolumn{4}{|c|}{ Optical data } \\
\hline HD 68456 & F6 & 1.00 & Anderson et al. (2010) \\
\hline 59 Vir & G0 & 0.19 & Linsky et al. (1994) (see Saar, 1996b) \\
\hline & & 0.42 & Anderson et al. (2010) (one temperature) \\
\hline & & $<0.30$ & Anderson et al. (2010) (cool spot solution) \\
\hline 58 Eri & G1 & 0.20 & Rüedi et al. (1997) \\
\hline$\kappa$ Cet & G5 & 0.36 & Saar and Baliunas (1992) \\
\hline 61 Vir & G6 & $<0.15$ & Anderson et al. (2010) \\
\hline \multirow[t]{3}{*}{$\xi$ Boo A } & G8 & 0.48 & Basri and Marcy (1988) \\
\hline & & 0.35 & Marcy and Basri (1989) \\
\hline & & 0.34 & Linsky et al. (1994) (see Saar, 1996b) \\
\hline $70 \mathrm{Oph}$ A & K0 & 0.22 & Marcy and Basri (1989) \\
\hline 40 Eri A & K1 & 0.06 & Rüedi et al. (1997) \\
\hline $36 \mathrm{Oph}$ B & K1 & 0.12 & Rüedi et al. (1997) \\
\hline \multirow[t]{3}{*}{$\epsilon$ Eri } & $\mathrm{K} 2$ & 0.35 & Basri and Marcy (1988) \\
\hline & & 0.30 & Marcy and Basri (1989) \\
\hline & & 0.17 & Rüedi et al. (1997) \\
\hline HD 166620 & $\mathrm{~K} 2$ & 0.23 & Basri and Marcy (1988) \\
\hline HD 17925 & $\mathrm{~K} 2$ & 0.25 & Saar (1996b) \\
\hline 36 Oph A & $\mathrm{K} 2$ & 0.20 & Marcy and Basri (1989) \\
\hline HR 222 & $\mathrm{~K} 2.5$ & 0.19 & Marcy and Basri (1989) \\
\hline HR 5568 & $\mathrm{~K} 4$ & 0.16 & Rüedi et al. (1997) \\
\hline EQ Vir & K5 & 1.38 & Saar (1996b) \\
\hline 61 Cyg A & K5 & 0.29 & Marcy and Basri (1989) \\
\hline$\epsilon$ Ind & K5 & 0.09 & Rüedi et al. (1997) \\
\hline
\end{tabular}


Table 2: Average magnetic fields from Stokes I in M-dwarfs.

\begin{tabular}{|c|c|c|c|c|}
\hline Star & Other Name & SpType & $B f[\mathrm{kG}]$ & Reference \\
\hline Gl 182 & & M0.0 & 2.5 & Reiners and Basri (2009) \\
\hline Gl 803 & AU Mic & M1.0 & 2.3 & Saar (1994) \\
\hline Gl 569A & & M2.0 & 1.8 & Reiners and Basri (2009) \\
\hline Gl 494 & DT Vir & $\mathrm{M} 2.0$ & 1.5 & Saar (1996b) \\
\hline Gl 70 & & M2.0 & $<0.2$ & Reiners and Basri (2007) \\
\hline \multirow[t]{2}{*}{ Gl 873} & EV Lac & M3.5 & 3.9 & Johns-Krull and Valenti $(1996,2000)$ \\
\hline & & & 3.7 & Saar (1994) \\
\hline \multirow[t]{2}{*}{ Gl 729} & & M3.5 & 2.0 & Johns-Krull and Valenti $(1996,2000)$ \\
\hline & & & 2.2 & Reiners and Basri (2007) \\
\hline Gl 87 & & M3.5 & 3.9 & Reiners and Basri (2007) \\
\hline \multirow[t]{5}{*}{ Gl 388} & AD Leo & M3.5 & 2.8 & Saar and Linsky (1985) \\
\hline & & & 2.6 & Saar (1994) \\
\hline & & & 3.3 & Johns-Krull and Valenti (2000) \\
\hline & & & 2.9 & Reiners and Basri (2007) \\
\hline & & & 3.2 & Kochukhov et al. (2009) \\
\hline GJ 3379 & & M3.5 & 2.3 & Reiners et al. (2009a) \\
\hline GJ 2069 B & & M4.0 & 2.7 & Reiners et al. (2009a) \\
\hline Gl 876 & & M4.0 & $<0.2$ & Reiners and Basri (2007) \\
\hline GJ $1005 \mathrm{~A}$ & & M4.0 & $<0.2$ & Reiners and Basri (2007) \\
\hline Gl 490 B & G $164-31$ & M4.0 & 3.2 & Phan-Bao et al. (2009) \\
\hline Gl 493.1 & & M4.5 & 2.1 & Reiners et al. (2009a) \\
\hline GJ 4053 & LHS 3376 & M4.5 & 2.0 & Reiners et al. (2009a) \\
\hline GJ 299 & & $\mathrm{M} 4.5$ & 0.5 & Reiners and Basri (2007) \\
\hline GJ 1227 & & M4.5 & $<0.2$ & Reiners and Basri (2007) \\
\hline GJ 1224 & & $\mathrm{M} 4.5$ & 2.7 & Reiners and Basri (2007) \\
\hline \multirow[t]{3}{*}{ Gl 285} & YZ CMi & M4.5 & 3.3 & Johns-Krull and Valenti (2000) \\
\hline & & & $>3.9$ & Reiners and Basri (2007) \\
\hline & & & 4.5 & Kochukhov et al. (2009) \\
\hline GJ $1154 \mathrm{~A}$ & & M5.0 & 2.1 & Reiners et al. (2009a) \\
\hline GJ 1156 & & M5.0 & 2.1 & Reiners et al. (2009a) \\
\hline Gl 905 & & M5.5 & $<0.1$ & Reiners and Basri (2007) \\
\hline GJ 1057 & & M5.0 & $<0.2$ & Reiners and Basri (2007) \\
\hline Gl 905 & & M5.5 & $<0.1$ & Reiners and Basri (2007) \\
\hline GJ 1245B & & M5.5 & 1.7 & Reiners and Basri (2007) \\
\hline GJ 1286 & & M5.5 & 0.4 & Reiners and Basri (2007) \\
\hline GJ 1002 & & M5.5 & $<0.2$ & Reiners and Basri (2007) \\
\hline \multirow[t]{2}{*}{ Gl 406} & & M5.5 & 2.4 & Reiners and Basri (2007) \\
\hline & & & $2.1-2.4$ & Reiners et al. (2007) \\
\hline Gl $412 \mathrm{~B}$ & & M6.0 & $>3.9$ & Reiners et al. (2009a) \\
\hline GJ 1111 & & M6.0 & 1.7 & Reiners and Basri (2007) \\
\hline Gl $644 \mathrm{C}$ & VB 8 & M7.0 & 2.3 & Reiners and Basri (2007) \\
\hline GJ 3877 & LHS 3003 & M7.0 & 1.5 & Reiners and Basri (2007) \\
\hline $2 \mathrm{M} 0440232-053008$ & & M7.0 & 1.6 & Reiners and Basri (2010) \\
\hline $2 \mathrm{M} 0741068+173845$ & & M7.0 & 1.0 & Reiners and Basri (2010) \\
\hline $2 \mathrm{M} 0752239+161215$ & & M7.0 & 3.5 & Reiners and Basri (2010) \\
\hline $2 \mathrm{M} 0818580+233352$ & & M7.0 & 1.0 & Reiners and Basri (2010) \\
\hline 2M 1048126-112009 & GJ 3622 & M7.0 & 0.6 & Reiners and Basri (2010) \\
\hline $2 \mathrm{M} 1356414+434258$ & & M7.0 & 2.7 & Reiners and Basri (2010) \\
\hline $2 \mathrm{M} 1456383-280947$ & & M7.0 & 1.2 & Reiners and Basri (2010) \\
\hline $2 \mathrm{M} 1534570-141848$ & & M7.0 & 2.0 & Reiners and Basri (2010) \\
\hline LHS 2645 & & M7.5 & 2.1 & Reiners and Basri (2007) \\
\hline
\end{tabular}


2M 0331302-304238 2M 0351000-005244 2M 0417374-080000 2M 0429184-312356A 2M 1006319-165326 2M 1246517+314811 2M 1253124+403403 2M 1332244-044112 2M 1546054+374946 LP 412-31

VB 10

2M 0248410-165121

2M 0320596+185423

2M 0517376-334902

2M 0544115-243301

2M $1016347+275149$ 2M 1024099+181553 2M 1141440-223215 2M 1309218-233035 2M 1440229+133923 $2 \mathrm{M} \mathrm{1843221+404021}$ 2M 2037071-113756 2M 2306292-050227 2M 2349489+122438 2M 0024442-270825B 2M 0306115-364753 $2 \mathrm{M} 1124048+380805$ 2M 1403223+300754 2M 2226443-750342 2M 2331217-274949 2M 2353594-083331 LHS 2924

LHS 2065

$2 \mathrm{M} 0019457+521317$ 2M 0109511-034326 2M 0334114-495334 2M 0443376+000205 2M 0853362-032932 2M 1048147-395606 2M 1224522-123835 $2 \mathrm{M} 1438082+640836$ 2M 2237325+392239
M7.5

M7.5

M7.5

M7.5

M7.5

M7.5

M7.5

M7.5

M7.5

M8.0

M8.0

M8.0

M8.0

M8.0

M8.0

M8.0

M8.0

M8.0

M8.0

M8.0

M8.0

M8.0

M8.0

M8.0

M8.5

M8.5

M8.5

M8.5

M8.5

M8.5

M8.5

M9.0

M9.0

M9.0

M9.0

M9.0

M9.0

M9.0

M9.0

M9.0

M9.5

M9.5
2.0 Reiners and Basri (2010)

1.4 Reiners and Basri (2010)

1.8 Reiners and Basri (2010)

2.5 Reiners and Basri (2010)

1.6 Reiners and Basri (2010)

$<0.4$ Reiners and Basri (2010)

1.6 Reiners and Basri (2010)

1.6 Reiners and Basri (2010)

2.7 Reiners and Basri (2010)

$>3.9$ Reiners and Basri (2007)

1.3 Reiners and Basri (2007)

1.4 Reiners and Basri (2010)

3.7 Reiners and Basri (2010)

1.6 Reiners and Basri (2010)

1.2 Reiners and Basri (2010)

2.1 Reiners and Basri (2010)

$<1.4$ Reiners and Basri (2010)

1.8 Reiners and Basri (2010)

1.2 Reiners and Basri (2010)

$<0.6$ Reiners and Basri (2010)

1.2 Reiners and Basri (2010)

$<0.2$ Reiners and Basri (2010)

0.6 Reiners and Basri (2010)

1.2 Reiners and Basri (2010)

2.1 Reiners and Basri (2010)

1.6 Reiners and Basri (2010)

2.0 Reiners and Basri (2010)

2.1 Reiners and Basri (2010)

1.8 Reiners and Basri (2010)

3.1 Reiners and Basri (2010)

2.0 Reiners and Basri (2010)

1.6 Reiners and Basri (2007)

$>3.9$ Reiners and Basri (2007)

3.7 Reiners and Basri (2010)

1.4 Reiners and Basri (2010)

1.4 Reiners and Basri (2010)

$<1.0$ Reiners and Basri (2010)

2.9 Reiners and Basri (2010)

2.3 Reiners and Basri (2010)

1.4 Reiners and Basri (2010)

1.2 Reiners and Basri (2010)

1.0 Reiners and Basri (2010) 
Table 3: Average magnetic fields from Stokes I in pre-main sequence stars and young brown dwarfs.

\begin{tabular}{|c|c|c|c|}
\hline Star & SpType & $B f[\mathrm{kG}]$ & Reference \\
\hline TAP 10 & G5 & $<0.7$ & Basri et al. (1992) \\
\hline GW Ori & G5 & $<1.0$ & Guenther et al. (1999) \\
\hline \multirow[t]{2}{*}{$\mathrm{T}$ Tau } & K0 & 2.4 & Johns-Krull (2007) \\
\hline & & 2.5 & Guenther et al. (1999) \\
\hline TAP 35 & K1 & 1.0 & Basri et al. (1992) \\
\hline 2MASS 05361049-0519449 & K3 & 2.31 & Yang and Johns-Krull (2011) \\
\hline V1735 Orig & K4 & 2.08 & Yang and Johns-Krull (2011) \\
\hline $\mathrm{LkCa} 15$ & K5 & 1.55 & Guenther et al. (1999) \\
\hline V1227 Ori & K5-K6 & 2.14 & Yang and Johns-Krull (2011) \\
\hline OV Ori & K5-K6 & 1.85 & Yang and Johns-Krull (2011) \\
\hline GI Tau & $\mathrm{K} 6$ & 2.7 & Johns-Krull (2007) \\
\hline TW Hya & K7 & 2.6 & Johns-Krull (2007) \\
\hline GK Tau & $\mathrm{K} 7$ & 2.3 & Johns-Krull (2007) \\
\hline GM Aur & K7 & 1.0 & Johns-Krull (2007) \\
\hline Hubble 4 & K7 & 2.5 & Johns-Krull et al. (2004) \\
\hline AA Tau & $\mathrm{K} 7$ & 2.8 & Johns-Krull (2007) \\
\hline \multirow[t]{2}{*}{ BP Tau } & K7 & 2.2 & Johns-Krull (2007) \\
\hline & & 2.6 & Johns-Krull et al. (1999b) \\
\hline DK Tau & K7 & 2.6 & Johns-Krull (2007) \\
\hline GG TauA & $\mathrm{K} 7$ & 1.2 & Johns-Krull (2007) \\
\hline TWA $9 \mathrm{~A}$ & K7 & 2.9 & Yang et al. (2008) \\
\hline TW Hya & K7 & 2.7 & Yang et al. (2008) \\
\hline V1568 Ori & $\mathrm{K} 7$ & 1.42 & Yang and Johns-Krull (2011) \\
\hline DG Tau & K7.5 & 2.6 & Johns-Krull (2007) \\
\hline 2MASS 05353126-0518559 & K8 & 2.84 & Yang and Johns-Krull (2011) \\
\hline V1348 Ori & K8 & 3.14 & Yang and Johns-Krull (2011) \\
\hline V1123 Ori & $\mathrm{M} 0 / \mathrm{K} 8$ & 2.51 & Yang and Johns-Krull (2011) \\
\hline DN Tau & M0 & 2.0 & Johns-Krull (2007) \\
\hline LO Ori & M0 & 3.45 & Yang and Johns-Krull (2011) \\
\hline LW Ori & M0.5 & 1.30 & Yang and Johns-Krull (2011) \\
\hline 2MASS 05350475-0526380 & M0.5 & 2.79 & Yang and Johns-Krull (2011) \\
\hline V568 Ori & M1 & 1.53 & Yang and Johns-Krull (2011) \\
\hline 2MASS 05351281-0520436 & M1 & 1.70 & Yang and Johns-Krull (2011) \\
\hline CY Tau & M1 & 1.2 & Johns-Krull (2007) \\
\hline DF Tau & M1 & 2.9 & Johns-Krull (2007) \\
\hline TWA 9B & M1 & 3.3 & Yang et al. (2008) \\
\hline DH Tau & M1.5 & 2.7 & Johns-Krull (2007) \\
\hline TWA 5A & M1.5 & $4.2^{\mathrm{a}}$ & Yang et al. (2008) \\
\hline V1124 Ori & M1.5 & 2.09 & Yang and Johns-Krull (2011) \\
\hline DE Tau & $\mathrm{M} 2$ & 1.1 & Johns-Krull (2007) \\
\hline TWA 8A & M2 & 2.7 & Yang et al. (2008) \\
\hline TWA 7 & M3 & 2.0 & Yang et al. (2008) \\
\hline UpSco 55 & M5.5 & 2.3 & Reiners et al. (2009b) \\
\hline CFHT-BD-Tau 4 & M7 & $<1.8$ & Reiners et al. (2009b) \\
\hline UpSco-DENIS 160603 & M7.5 & $<0.4$ & Reiners et al. (2009b) \\
\hline 2MASS 1207 & M8 & $<1.0$ & Reiners et al. (2009b) \\
\hline$\rho$-Oph-ISO 32 & M8 & $<2.4$ & Reiners et al. (2009b) \\
\hline
\end{tabular}


Table 4: Magnetic field measurements not listed in Tables 1, 2, and 3.

\begin{tabular}{ll}
\hline Publication & Comment \\
\hline Robinson et al. (1980) & $\xi$ Boo A, 70 Oph A, 61 Vir \\
Vogt (1980) & BY Dra, HD 88230, 61 Cyg A, HD 209813 \\
Marcy (1981) & $\xi$ Boo A \\
Golub et al. (1983) & $\lambda$ And \\
Marcy (1984) & 29 G- and K main sequence stars \\
Marcy and Bruning (1984) & 8 evolved stars \\
Gray (1984) & 18 F-, G-, and K-dwarfs \\
Gondoin et al. (1985) & $\xi$ Boo A, 61 UMa, $\lambda$ And \\
Saar et al. (1986) & EQ Vir \\
Bruning et al. (1987) & 7 K- and M-dwarfs \\
Mathys and Solanki (1989) & Preliminary results, "Stenflo-Lindegren" technique \\
Bopp et al. (1989) & VY Ari \\
Saar (1990) & Selection of 31 G-M star measurements; including unpublished data \\
\hline
\end{tabular}




\subsection{Longitudinal fields and Zeeman Doppler maps from Stokes V}

\subsubsection{Dwarfs and subgiants}

Advantages and caveats in searching for cool star magnetism through polarization measurements were discussed in the Sections above. For a detection, the detailed line shape in the unpolarized case does not have to be understood at very high level, which means that the signal of a potential field will be relatively straightforward to detect (given suitable instrumentation). The signal expected from magnetic cool stars, however, may be extremely weak because of flux cancellation and complicated field geometries.

Early programs to search for longitudinal fields in late-type stars were presented by Brown and Landstreet (1981) and Borra et al. (1984). Both works show sophisticated methods to search for circular polarization simultaneously in many spectral lines and obtain encouraging results in hot stars with known strong magnetic fields. Both programs, however, fail to detect polarization signals in late-type stars confirming the suspicion that net longitudinal fields are difficult to detect in these stars. The key idea in the two mentioned programs is to co-add information from many spectral lines in order to enhance the polarization signal. Masks transmitting only the light in the vicinity of stellar absorption lines are used so that the final signal is constructed to be something like an average signal from many lines. The basic idea is very similar to the construction of a mean line profile using the approach of Least Squares Deconvolution (Section 2.1.8).

A detection of circular polarization in a low-mass star was successful in an effort to create Zeeman Doppler Images in RS CVn binaries. Donati et al. (1990) show signals in circular polarization in three Fe I lines of HR 1099, and more successful detections in RS CVn's are presented in Donati et al. (1992). These works obtained very high SNR data in order to detect polarization signals in individual lines. Later, the technique of Least Squares Deconvolution entered the domain of polarization measurements and Zeeman Doppler Imaging (see Section 2.1) and, since then, Stokes V signatures were investigated in many different stars (e.g., Donati et al., 1997). Table 5 gives a summary of Stokes $\mathrm{V}$ measurements in cool dwarfs and subgiants. It is sometimes difficult to compare the results from different projects, because results are sometimes presented in the form of surface maps and sometimes in the form of average magnetic field strengths. Note that also in this context, average fields mean the average value for the detected unsigned magnetic field, $|B|$, a definition similar to the value measured in Stokes I; the average value of the signed magnetic field is zero by construction.

A tremendous amount of work was put into the analysis of magnetic geometries in stars through ZDI, and the possibility of reconstructing magnetic fields on stellar surfaces is truly amazing. As laid out in Section 2.1, however, the interpretation of the field maps is very difficult, and conclusions have to be drawn with great care. Typical average field values for sun-like stars of spectral types $\mathrm{F}-\mathrm{K}$ are of the order of ten Gauss, local field strengths in Doppler maps go up to several hundred Gauss. Note that most of the stars imaged with ZDI are rapid rotators that are much more active than the slow-rotating Sun.

Much work was done also on maps of magnetic fields in M stars. Results derived from time-series of Stokes V measurements are presented in Donati et al. (2008b) and Morin et al. (2008, 2010). The typical average magnetic field strengths found in Stokes V measurements of M dwarfs are significantly larger than average fields from Stokes V work found in hotter, sun-like stars. Average fields up to $1.5 \mathrm{kG}$ were detected, and the range of $|B|$ in Doppler maps from Stokes $\mathrm{V}$ extend up to $2 \mathrm{kG}$, a value at which the weak-field approximation probably approaches its limitations.

Kochukhov et al. (2011) presented very high quality measurements of all four Stokes parameters in three sun-like stars. Using LSD, they detect circular and linear polarization in the RS CVn binary HR 1099. For the first time, linear polarization is detected in sun-like stars. Fields between 10 and $25 \mathrm{G}$ are found in different observations of HR 1099. The signal detected in linear polarization is significantly more complex than the circular polarization signal and, as expected, linear polarization is weaker than circular polarization by roughly a factor 10 . 


\subsubsection{Giants}

Another class of stars in the focus of magnetic field research are giants. Some of these evolved stars can be rather active, and they possess large convection zones potentially allowing the operation of a dynamo. Stokes V observing campaigns are available in a handful of giants providing information about their magnetism. For the active FK Com star HD 199178, Petit et al. (2004) constructed Zeeman Doppler maps with field strengths up to several hundred Gauss. The other work summarized in Table 6 find mean fields that are on the order of $1 \mathrm{G}$ or below, this means they are on average much weaker than the fields found in dwarfs.

\subsubsection{Young stars}

Pre-main sequence stars are particularly interesting objects for magnetic field measurements because fields may be of fossil origin or generated through dynamo operation, and magnetism is required for magnetospheric accretion (see Section 3.1.3). The observational situation from polarization measurements is similar to the one in main-sequence stars: results are available in the form of magnetic Doppler maps providing a range and geometry of the detected net field, and there are results reporting average field values from multiple or single polarization measurements.

A summary of magnetic field reports from circular polarization measurements in pre-main sequence stars is given in Table 7. Field strengths up to several hundred Gauss, and in the case of BP Tau up to $3 \mathrm{kG}$ are found in Zeeman Doppler maps from photospheric lines. Average fields on the order of ten to several hundred Gauss have been found in the analysis of photospheric lines. A remarkable difference exists between the field strengths found in polarization measurements carried out in photospheric lines and those carried out in emission lines that are formed predominantly in the region of the accretion shock, usually the He I line at $5876 \AA$. In the latter, field strengths are on the order of a few kilo-Gauss similar to field strengths detected in Stokes I measurements.

Assuming the same flux values across the star, the net flux seen in the accretion region alone may be higher than the net flux averaged over the photosphere, which would mean that the difference is a purely geometric effect. The accretion column could stem from a more or less unipolar bundle of fluxtubes allowing to see the true (almost uncanceled) flux, while the photospheric lines come from the entire star and are subject to cancellation. Furthermore, field strengths may actually be higher in the region of accretion, and the differences between magnetic fields measurements from integrated light and results from circular polarization in photospheric and accretion lines may provide additional information on the magnetic field geometry in pre-main sequence stars (see e.g., Johns-Krull, 2007; Donati et al., 2011c, and references therein). We come back to this point in Section 6.

Living Reviews in Solar Physics

http://www. livingreviews.org/lrsp-2012-1 
Table 5: Longitudinal magnetic fields or Zeeman Doppler maps from Stokes V for dwarfs and subgiants.

\begin{tabular}{|c|c|c|c|c|c|}
\hline Star & Other Name & SpType & $\begin{array}{c}<\boldsymbol{B}> \\
{[\mathrm{G}]}\end{array}$ & $\boldsymbol{B}$ Range & Reference \\
\hline$\tau$ Boo & & F7V & & $0-3$ & Catala et al. (2007) \\
\hline HR 1817 & & $\mathrm{~F} 8 \mathrm{~V}$ & 13 & $0-250$ & Marsden et al. (2006b) \\
\hline HD 73350 & & G0V & $12^{\mathrm{b}}$ & $0-20$ & Petit et al. (2008) \\
\hline Sun $^{\mathrm{a}}$ & & $\mathrm{G} 2 \mathrm{~V}$ & 4 & & Daou et al. (2006) \\
\hline$\alpha$ Cen A & & $\mathrm{G} 2 \mathrm{~V}$ & $<0.2$ & & Kochukhov et al. (2011) \\
\hline \multirow[t]{2}{*}{ HD 171488} & & $\mathrm{G} 2 \mathrm{~V}$ & & $0-500$ & Marsden et al. (2006a) \\
\hline & & & 31 & $0-500$ & Marsden et al. (2006b) \\
\hline HD 146233 & & $\mathrm{G} 2 \mathrm{~V}$ & 3.6 & $0-5$ & Petit et al. (2008) \\
\hline HD 76151 & & G3V & 5.6 & $0-10$ & Petit et al. (2008) \\
\hline HD 190771 & & G5IV & $15^{\mathrm{b}}$ & $0-20$ & Petit et al. (2008) \\
\hline LQ Hya & & $\mathrm{K} 0 \mathrm{~V}$ & & $0-800$ & Donati (1999) \\
\hline AB Dor & & $\mathrm{K} 0 \mathrm{~V}$ & & $0-800$ & Donati et al. (1999) \\
\hline HD 46375 & & $\mathrm{~K} 0 \mathrm{~V}$ & & $0-5$ & Gaulme et al. (2010) \\
\hline II Peg & & K1IV & & $0-700$ & Carroll et al. (2007) \\
\hline \multirow[t]{2}{*}{ HR 1099} & & K1IV & & $0-800$ & Donati (1999) \\
\hline & & & $12-25$ & & Kochukhov et al. (2011) \\
\hline HD 189733 & & $\mathrm{~K} 2 \mathrm{~V}$ & & $0-40$ & Moutou et al. (2007) \\
\hline$\epsilon$ Eri & & $\mathrm{K} 2 \mathrm{~V}$ & $-6-5$ & & Kochukhov et al. (2011) \\
\hline Gl 890 & & MOV & $<18$ & & Phan-Bao et al. (2009) \\
\hline Gl 410 & DS Leo & $\mathrm{M} 0 \mathrm{~V}$ & 100 & & Donati et al. (2008b) \\
\hline Gl 182 & & $\mathrm{M} 0.5 \mathrm{~V}$ & 172 & & Donati et al. (2008b) \\
\hline Gl 494 & DT Vir & $\mathrm{M} 0.5 \mathrm{~V}$ & 150 & & Donati et al. (2008b) \\
\hline Gl 49 & & M1.5 & 27 & & Donati et al. (2008b) \\
\hline GJ 9520 & OT Ser & M1.5 & 130 & & Donati et al. (2008b) \\
\hline Gl 569 A & CE Boo & $\mathrm{M} 2.0 \mathrm{~V}$ & 103 & & Donati et al. (2008b) \\
\hline Gl 752 A & LHS 473 & $\mathrm{M} 2.5 \mathrm{~V}$ & 16 & & Phan-Bao et al. (2009) \\
\hline GJ 3241 & KP Tau & M3V & 100 & & Phan-Bao et al. (2009) \\
\hline Gl 388 & AD Leo & M3V & 185 & & Morin et al. (2008) \\
\hline Gl $896 \mathrm{~A}$ & EQ Peg A & $\mathrm{M} 3.5 \mathrm{~V}$ & 480 & & Morin et al. (2008) \\
\hline \multirow[t]{2}{*}{ Gl 873} & EV Lac & $\mathrm{M} 3.5 \mathrm{~V}$ & $18-40$ & & Phan-Bao et al. (2006) \\
\hline & & & 530 & & Morin et al. (2008) \\
\hline \multirow[t]{2}{*}{ GJ 4247} & V374 Peg & $\mathrm{M} 4 \mathrm{~V}$ & & $0-2000$ & Donati et al. (2006) \\
\hline & & & 710 & & Morin et al. (2008) \\
\hline Gl $490 \mathrm{~B}$ & G $164-31$ & $\mathrm{M} 4 \mathrm{~V}$ & 680 & $0-1800$ & Phan-Bao et al. (2009) \\
\hline Gl 285 & YZ CMi & $\mathrm{M} 4.5 \mathrm{~V}$ & 555 & & Morin et al. (2008) \\
\hline \multirow[t]{2}{*}{ Gl 896 B } & EQ Peg B & $\mathrm{M} 4.5 \mathrm{~V}$ & 450 & & Morin et al. (2008) \\
\hline & & & 290 & & Phan-Bao et al. (2009) \\
\hline 2E 4498 & $2 \mathrm{E} 4498$ & $\mathrm{M} 4.5 \mathrm{~V}$ & 440 & & Phan-Bao et al. (2009) \\
\hline Gl 51 & & M5V & 1500 & & Morin et al. (2010) \\
\hline GJ 1156 & & M5V & 100 & & Morin et al. (2010) \\
\hline GJ 1245 B & & M5.5V & 150 & & Morin et al. (2010) \\
\hline Gl 905 & HH And & $\mathrm{M} 5.5 \mathrm{~V}$ & $<5$ & & Phan-Bao et al. (2006) \\
\hline Gl 412 B & WX UMa & $\mathrm{M} 6 \mathrm{~V}$ & 1000 & & Morin et al. (2010) \\
\hline GJ 1111 & DX Cnc & M6V & 100 & & Morin et al. (2010) \\
\hline GJ 3622 & & M6.5V & 55 & & Morin et al. (2010) \\
\hline
\end{tabular}


Table 6: Longitudinal magnetic fields or Zeeman Doppler maps from Stokes V for giants.

\begin{tabular}{|c|c|c|c|c|}
\hline Star & SpType & $\begin{array}{c}<B> \\
{[G]}\end{array}$ & $\begin{array}{c}B \text { Range } \\
{[\mathrm{G}]}\end{array}$ & Reference \\
\hline Betelgeuse & M2Iab & $0.5-1.6$ & & Aurière et al. (2010) \\
\hline HD 199178 & G5III & & $0-600$ & Petit et al. (2004) \\
\hline V390 Aur & G8III & $5-15$ & & Konstantinova-Antova et al. (2008) \\
\hline Pollux & KOIII & $0.1-1.4$ & & Aurière et al. (2009) \\
\hline Arcturus & K1.5III & $0.4-0.7$ & & Sennhauser and Berdyugina (2011) \\
\hline EK Boo & M5III & $0.1-0.8$ & & Konstantinova-Antova et al. (2010) \\
\hline
\end{tabular}

Table 7: Longitudinal magnetic fields or Zeeman Doppler maps from circular polarization in pre-main sequence stars.

\begin{tabular}{|c|c|c|c|c|}
\hline Star & SpType & $\begin{array}{c}<\boldsymbol{B}> \\
{[\mathrm{G}]}\end{array}$ & $\begin{array}{c}\boldsymbol{B} \text { Range } \\
{[\mathrm{G}]}\end{array}$ & Reference \\
\hline HD $155555 \mathrm{~A}$ & G5 & & $0-500$ & Dunstone et al. (2008) \\
\hline CV Cha & G8 & & $0-700$ & Hussain et al. (2009) \\
\hline HD $155555 \mathrm{~B}$ & K0 & & $0-300$ & Dunstone et al. (2008) \\
\hline T Tau & K0 & 12 & & Daou et al. (2006) \\
\hline CR Cha & $\mathrm{K} 2$ & & $0-400$ & Hussain et al. (2009) \\
\hline V410 Tau & K4 & & $0-1000$ & Skelly et al. (2010) \\
\hline \multirow[t]{2}{*}{ V2129 Oph } & K5 & & $0-800$ & Donati et al. (2007) \\
\hline & & & $0-2000$ & Donati et al. (2011a) \\
\hline \multirow[t]{3}{*}{ Tw Hya } & K6 & 150 & & Yang et al. (2007) \\
\hline & & $2000^{\mathrm{a}}$ & & Yang et al. (2007) \\
\hline & & & $0-3000$ & Donati et al. (2011b) \\
\hline \multirow[t]{4}{*}{ BP Tau } & K7 & 500 & $0-3000$ & Donati et al. (2008a) \\
\hline & & $2750^{\mathrm{a}}$ & & Johns-Krull and Valenti (2000) \\
\hline & & 200 & & Johns-Krull et al. (1999a) \\
\hline & & $2500^{\mathrm{a}}$ & & Johns-Krull et al. (1999a) \\
\hline DK Tau & M0 & $1450^{\mathrm{a}}$ & & Johns-Krull and Valenti (2000) \\
\hline AA Tau & M0 & $2900^{\mathrm{a}}$ & & Johns-Krull and Valenti (2000) \\
\hline DF Tau & M2 & $1000^{\mathrm{a}}$ & & Johns-Krull and Valenti (2000) \\
\hline V2247 Oph & M2.5 & & $0-800$ & Donati et al. (2010) \\
\hline
\end{tabular}




\section{The Rotation-Magnetic Field-Activity Relation}

The generation of stellar magnetic fields is the result of complex mechanisms acting in the moving plasma of the stellar interior. The variety of magnetic field-related phenomena observed on the Sun should be explainable by a theory of the solar dynamo, but this dynamo continues to pose serious challenges to both observers and theoreticians. Although our knowledge about the solar magnetic properties and its time-dependence is rich and growing with the growing fleet of instrumentation observing the magnetic Sun, this single star can only exhibit magnetic features according to its own properties, and the investigation of the Sun alone will not lead to a full understanding of stellar dynamos in general. It is, therefore, of large interest for a deep understanding of stellar dynamos and, in particular, of the solar dynamo to understand the dependence of magnetic field generation on stellar properties.

The driving force of dynamos operating in the Sun and low-mass stars is the interplay between convective plasma motion, density and temperature stratification, and stellar rotation. Differential rotation and shear play particularly important roles in the most favored versions of sun-like dynamos. For overviews on the solar dynamo and theoretical backgrounds, I refer to the many reviews on this topic, for example Ossendrijver (2003) and Charbonneau (2010). One often-mentioned expectation from stellar dynamo models is the relation between the magnetic field strength and the rotation of a star. The relation is expected because the efficiency of a dynamo can be described by the "dynamo number", $D$, that is related to the so-called $\alpha$-effect that itself depends on rotation. The exact functional dependence between dynamo efficiency and rotation is difficult to assess, but one can argue that a dynamo can only exist if the rotational influence on convection, expressed as the Coriolis number Co, exceeds a certain value in order to create the differential rotation that is required for the dynamo process (see, e.g., Durney and Latour, 1978). The Coriolis number is proportional to the Rossby number that is often used in work on stellar activity, $R o=P_{\text {rot }} / \tau_{\text {conv }}$, with $P_{\text {rot }}$ the rotational period and $\tau_{\text {conv }}$ the convective overturn time.

The definition of the convective overturn time is not exactly well defined particularly in very low-mass stars (e.g., Gilliland, 1986; Kim et al., 1996). It has therefore been attempted to derive "empirical" turnover times assuming a relation between magnetic activity and stellar rotation. A first and very successful approach was presented by Noyes et al. (1984) who connected observations of stellar chromospheric activity and rotation. Investigating a rich sample of X-ray, coronal, activity measurements, Pizzolato et al. (2003) were able to show a well-defined rotation-activity relation connecting normalized X-ray luminosity and Rossby number. The latter is a mass-dependent function chosen to minimize the scatter in the rotation-activity relation (Figure 18). Empirical convective overturn times in low-mass stars were derived by Kiraga and Stẹpień (2007), and Barnes and Kim (2010) cover a wide range of masses.

It has been argued that the construction of a mass-dependent empirical convective overturn time in order to minimize scatter in the rotation-activity relation is in principle nothing else than a compensation for a dependence of activity on stellar luminosity (Basri, 1986; Pizzolato et al., 2003). In other words, while normalized activity seems to scale with Rossby number, total (unnormalized) flux seems to scale with rotation period. It is argued that the reason for this is the approximate scaling of $\tau_{\text {conv }}$ with $\left(L_{\mathrm{bol}}\right)^{-0.5}$. Nevertheless, a tight dependence between Rossby number and normalized activity is clearly observed in sun-like and early- to mid-M type stars. The result is that magnetic activity is rising with decreasing Rossby number as long as $R o \geq 0.1$. At $R o \approx 0.1$, activity saturates and does not grow further with decreasing Rossby number. This behavior is interpreted as increasing dynamo efficiency with faster rotation in the regime where rotation is not yet dominating convection $(R o \geq 0.1)$. This is what may be expected from the dynamo models introduced above. At fast rotation $(R o \approx 0.1)$ the dynamo reaches a level of saturation that cannot be exceeded even if the star is spinning much faster.

We know from the Sun that activity is caused by magnetic fields. Together with expectations of 

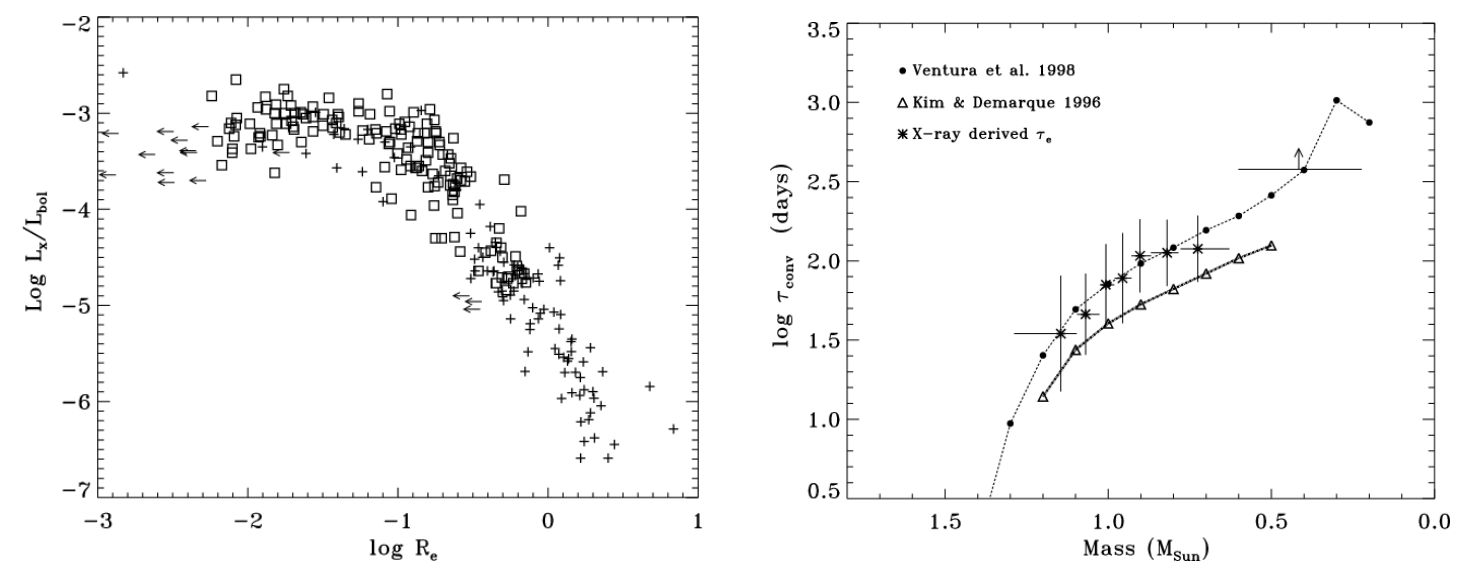

Figure 18: Left panel: Rotation-activity relation showing the normalized X-ray luminosity as a function of Rossby number. Right panel: Empirical turnover time chosen to minimize the scatter in the rotationactivity relation (from Pizzolato et al., 2003, reprinted with permission (C) ESO).

the relation between rotation and magnetic field strength from dynamo theory, it is straightforward to conclude that the reason for the observed rotation-activity relation is a rotation-dependence of magnetic field generation, i.e., what we observe is a direct consequence of the magnetic dynamo efficiency. From an observational standpoint, this is not entirely clear because all we have discussed so far is that activity scales with rotation (or Rossby number), but this may also be due to a constant magnetic field translating to observable activity in a fashion that depends on rotation. A direct link between rotation and magnetic field observations was shown by Saar (1996a, 2001), the observational basis of this work was discussed in Section 3.1. In sun-like and young stars it is found that magnetic field strengths indeed are a function of rotational period: $B f$ follows a relation that is proportional to some power of Ro consistent with expectations. In sun-like stars, however, magnetic fields can not be measured at very low Rossby numbers (saturated regime) because spectral line widths are too broad due to the rotational broadening at the corresponding rotation rates. Mainly because of the smaller radii, and perhaps also because of longer convective overturn times, the relation between Rossby number and equatorial velocity favors the detection of Zeeman splitting at low Rossby numbers (in the saturated regime) in low-mass stars (see, e.g., Reiners, 2007); M dwarfs have very small radii (and long overturn times) so that for small Rossby numbers the corresponding surface velocities are relatively low. This allows measuring magnetic fields of M stars well within the saturated regime. For M dwarfs of spectral type M6 and earlier, Reiners et al. (2009a) found that average magnetic fields indeed show evidence for saturation at low Rossby numbers. This can be interpreted as evidence that saturation of activity at high rotation rates is a consequence of saturation of the average magnetic field and that $B$ itself is limited (in contrast to a limit of the filling factor $f$ or of the coupling between magnetic fields and non-thermal heating). Unfortunately, it was not yet possible to separate $B$ from $f$ in the measurements of magnetic flux density $B f$ so that the true range and variation of both field strength $B$ and filling factor $f$ remains unknown. Although there is good evidence for a firm upper limit, there is still room for some variation of $B$ as a function of $R o$ in the saturated regime. Further observations, and especially information about values of both $B$ and $f$ are highly desired.

The relation between magnetic flux density and Rossby number is shown in Figure 19. Crosses are from sun-like stars and define the rising, unsaturated part of the rotation-activity relation, and circles are M-type stars defining saturation at a few kilo-Gauss average field strength. At least for sun-like stars and early-M stars, the rotation-magnetic field relation seems to be rather well defined. Looking back to the discussion on detectability of magnetic fields, at least in sun-like 


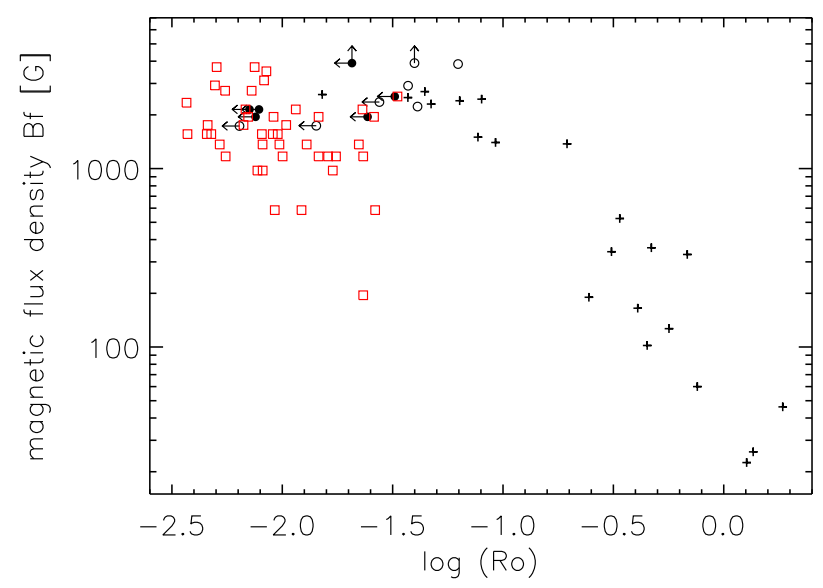

Figure 19: Magnetic fields as a function of Rossby number. Crosses are sun-like stars Saar (1996a, 2001), circles are M-type of spectral class M6 and earlier (see Reiners et al., 2009a). For the latter, no period measurements are available and Rossby numbers are upper limits (they may shift to the left hand side in the figure). The black crosses and circles follow the rotation-activity relation known from activity indicators. Red squares are objects of spectral type M7-M9 (Reiners and Basri, 2010) that do not seem to follow this trend $\left(\tau_{\text {conv }}=70 \mathrm{~d}\right.$ was assumed for this sample).

stars, some cautious doubt may be allowed as to the relation between Rossby number and $B f$ in the "low-field" regime $(B f<1 \mathrm{kG})$. First, in principle, the detection of magnetic fields in this region and, in particular, from optical data, is extremely difficult and the significance of the data points is difficult to assess (see Section 4.3). Second, assuming that the Sun has a Rossby number somewhere between 2 and $0.5\left(P_{\text {rot }} \sim 26 \mathrm{~d}, \tau_{\text {conv }} \sim 12-50 \mathrm{~d}\right)$, the average magnetic flux for the Sun is on the order of $20-100 \mathrm{G}$, which is significantly above the value detectable in the Sun if it is observed as a star.

One interesting and relatively firm conclusion from $\mathrm{M}$ dwarf magnetic field measurements is that the typical upper limit for average magnetic fields is of the order of a few kilo-Gauss, average fields of $10 \mathrm{kG}$ are not observed, and the upper limit does not seem to significantly depend on temperature. This contradicts the prediction of a close correlation between (maximum) magnetic field strength and spectral type introduced by assuming a limiting influence of buoyancy forces on the dynamo efficiency (Durney and Robinson, 1982). However, this conclusion is only valid for M-type main sequence stars because we have no good estimate of maximum field strengths in $\mathrm{F}$-K-type stars, and magnetic fields in pre-main sequence stars may follow different rules.

\subsection{The dynamo at very low masses}

Stars of spectral type $\mathrm{G}-\mathrm{K}$ are considered sun-like stars, their interior structure with an outer convective envelope and an inner radiative core, and the general observational evidence for similar evolutionary paths lead to the conclusion that this group of stars follows physical principles that are very much alike. Early-M type stars can be sorted into the same category. However, at midand late-M spectral types, serious changes occur to stellar structure that are predicted from theory and observed in different aspects of stellar evolution. The first important change in stellar structure occurs at spectral types M3/M4 (in field dwarfs). Stars hotter than M3 have a radiative core like the Sun, and cooler sun-like stars have convective envelopes that extend deeper into the interior of the star. On the other hand, stars cooler than M4 are believed to be fully convective without a 
radiative core, and without a transition region between the outer convective envelope and the inner radiative core. Because in solar dynamo models this transition region, the tachocline, is believed to be the locus where (at least the cyclic part of) the stellar dynamo is most efficient, a change in dynamo efficiency has been expected at spectral type M3/M4. So far, measurements of average field strengths show no evidence for such a break in dynamo behavior (but see Section 6); rapidly rotating stars on both sides of the convective boundary can produce magnetic fields of kG-strength.

Following stellar evolution to even lower temperatures and later spectral types leads into the regime of very-low-mass objects. At spectral type $\sim \mathrm{M} 6$ and later, there is no longer a unique relation between effective temperature and the mass of an object, because this is the regime where field stars and young brown dwarfs co-exist. Brown dwarfs are objects with mass lower than $\approx 0.08 M_{\odot}$ that cannot burn hydrogen into helium for significant fractions of their lifetime. The difference from stars to brown dwarfs is dramatic if long-term evolution is concerned. For a potential dynamo operating in objects at the cool end of the main-sequence, however, the source of energy is not necessarily expected to make any difference. Therefore, no fundamental difference should exist between magnetic fields on very-low mass stars and massive brown dwarfs. However, we have seen in Section 3.1.3 that so far no field could be detected in a brown dwarf.

Another effect that probably bears some importance for magnetic field generation in verylow mass stars is growing atmospheric neutrality (Meyer and Meyer-Hofmeister, 1999; Mohanty et al., 2002). Since magnetic fields can only couple to ions and electrons, the lack of ionization in atmospheres below a few thousand $\mathrm{K}$ should play a role in the generation of magnetic fields. The growth of ionisation fraction with depth may still allow field coupling sufficiently deep within the stellar interior and atmospheric ionisation may be provided by dust ionisation (see Helling et al., 2011). Growing atmospheric neutrality certainly is important for the coupling between magnetic fields and the stellar wind. Evidence for the latter is found in observations of high rotation velocities interpreted as very weak angular momentum loss in very-low mass stars and brown dwarfs (Reiners and Basri, 2008; Blake et al., 2010).

Recently, magnetic fields measurements in a sample of very-low mass stars of spectral types M7-M9 were reported in Reiners and Basri (2010). Stars in this regime are probably all fully convective and their atmospheres are significantly cooler than atmospheres in sun-like stars. The overall distribution of field strengths in very-low-mass stars does not seem to differ from highermass, earlier M dwarfs; average fields of up to $\sim 4 \mathrm{kG}$ are detected. However, there is evidence for a change in the relation between rotation and magnetic field strength. Reiners and Basri (2010) show that the correlation between projected surface velocity $v \sin i$ gradually weakens from spectral type M7 to M9 showing virtually no relation at lowest temperatures. We can estimate the Rossby number of the M7-M9 stars and plot the average magnetic fields of the M7-M9 stars as a function of Rossby number (red squares in Figure 19). This plot shows that the Rossby numbers for the sample stars are much lower than those for earlier stars. For given Rossby number, the distribution of average field strength $B f$ extends to lower values than seen in hotter stars. This also may be interpreted as evidence for the breakdown of the rotation-magnetic field relation in very-low mass stars.

\subsection{Magnetism and $\mathrm{H} \alpha$ activity}

The possibility to measure the surface magnetic fields in M stars from molecular FeH lines opens the opportunity to study the generation and consequences of magnetic fields in a large sample (see Table 2). The most frequently used FeH lines around $1 \mu \mathrm{m}$ can be observed with nearinfrared instrumentation, but also with spectrographs like HIRES or UVES that operate at optical wavelengths. With optical spectrographs, it is often possible to observe molecular FeH and simultaneously cover the most frequently used activity indicator in cool stars, the emission line of $\mathrm{H} \alpha$. Chromospheric emission is known to be variable on timescales of minutes and strictly simultane-

Living Reviews in Solar Physics

http://www. livingreviews.org/lrsp-2012-1 
ous observation of $\mathrm{H} \alpha$ together with the magnetic field is, therefore, particularly useful. Figure 20 shows the relation between normalized $\mathrm{H} \alpha$ luminosity $\log L_{\mathrm{H} \alpha} / L_{\mathrm{bol}}$ and the average magnetic field $B f$ for the $M$ dwarf measurements given in Table 2. A deeper discussion of this relation can be found in Reiners and Basri (2007, 2010).

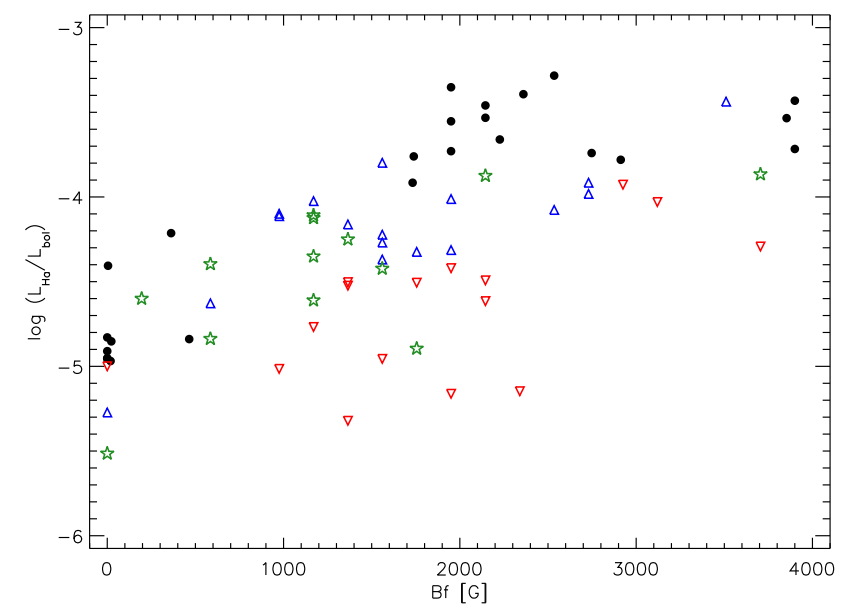

Figure 20: Normalized $\mathrm{H} \alpha$ luminosity as a function of $B f$. Filled black circles: Early-/mid-M type stars of spectral type M0 - M6; blue triangles: spectral type M7; green stars: spectral type M8; red upside down triangles: spectral type M9.

One can draw two interesting conclusions from the data shown in Figure 20. First, in early$\mathrm{M}$ dwarfs $(\leq \mathrm{M} 6)$, the relation between magnetic field and chromospheric activity follows a curve similar to the rotation-activity relation; chromospheric activity grows with average field strength in the low-field regime $(B f \leq 2000 \mathrm{G})$ but saturates at a critical field strength. The critical field strength in early M stars seems to be close to $2000 \mathrm{G}$. At this point, however, the data sample is rather sparse and uncertainties are high so that such conclusions can only be preliminary. This saturation - if existent - is different from the saturation of the field itself (at Ro $\sim 0.1$ ). The rotation-activity relation implies that fields cannot be stronger than $3-4 \mathrm{kG}$ in general. A saturation of chromospheric activity at $2000 \mathrm{G}$ would mean that additionally, $\mathrm{H} \alpha$ emission saturates at even lower rotation rates when the field is sufficiently strong.

Figure 20 also contains data for cooler stars of spectral type M7-M9. In these stars, the level of $\log L_{\mathrm{H} \alpha} / L_{\mathrm{bol}}$ is lower on average than in hotter stars, a reason may be the growing atmospheric neutrality that weakens the coupling between the ionized atmosphere and magnetic fields hence rendering magnetic heating ineffective. Interestingly, there is a hint in Figure 20 that the field strength at which saturation occurs may grow to larger fields in cooler stars. In other words, cooler stars need stronger field strengths to generate the same level of activity than hotter stars, and the saturation is not limited by a fixed field strength but by a maximum level of chromospheric emission $\left(\log L_{\mathrm{H} \alpha} / L_{\mathrm{bol}}\right)$.

\subsection{A posteriori knowledge about detectability of magnetic fields}

I have argued in Section 3.1 and 4 that the detection of magnetic fields from optical lines is extremely difficult, and that our picture of the non-saturated part of the rotation-magnetic field relation may be biased by influences of activity on line profiles other than the Zeeman effect. In other words, the relation between rotation period and measured fields may be driven by the 
presence of starspots that are generated when stars rotate more rapidly, and they influence the line profiles in a way that may mimic the presence of Zeeman splitting. It is currently very difficult to assess the influence of this effect. The rotation-magnetic field relation would probably look similar to the one shown in Figure 19, but its absolute values may differ significantly. One consequence of this might be the mismatch between the solar average field and the value predicted according to its rotation period.

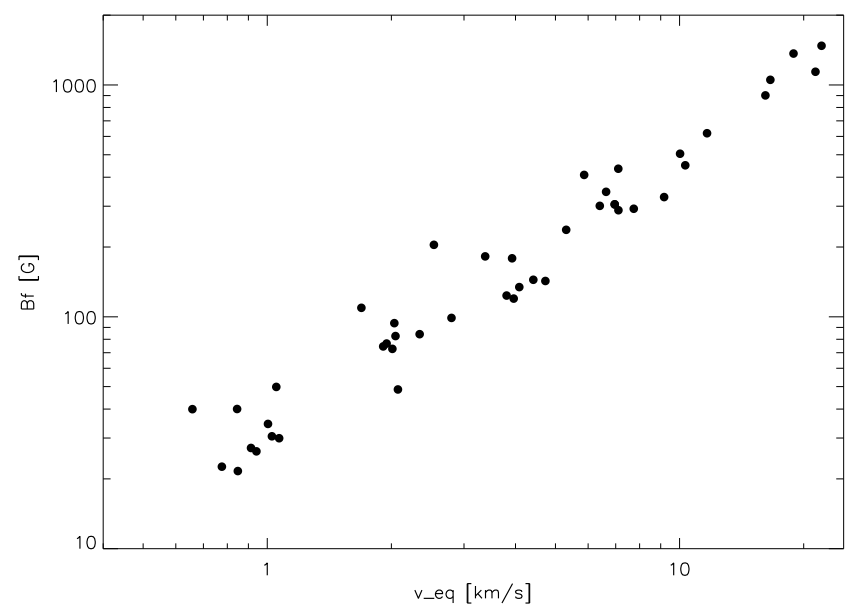

Figure 21: Estimated average magnetic fields as a function of equatorial rotational velocity. Equatorial velocities are calculated for stars with measured rotation periods in Noyes et al. (1984) and Donahue et al. (1996). Average fields are estimated from the relation given in the text.

As an interesting exercise, we can take the rotation-magnetic field relation in the unsaturated part of Figure 19 and estimate magnetic field strengths for a sample of stars with measured rotation periods. The relation in Figure 19 can be approximated by $B f=70 \mathrm{Ro}^{-1.5}$. We can then take empirical Rossby numbers (using measured rotation periods) from the work of Noyes et al. (1984) and Donahue et al. (1996), convert rotation period into approximate surface rotation velocity for each star, and estimate the average magnetic field strength from the relation between $B f$ and $R o$. The result of this exercise is shown in Figure 21. We can conclude that according to the relation in Figure 19, sun-like stars with surface rotation velocities of $5 \mathrm{~km} \mathrm{~s}^{-1}$ generate average magnetic fields of approximately $B f=200 \mathrm{G}$. Kilo-Gauss field strengths are generated in stars that rotate as rapidly as $v_{\text {eq }}=15 \mathrm{~km} \mathrm{~s}^{-1}$. A rough approximation to the relation shown in Figure 21 is $B f \approx 50 v_{\text {eq }}$, with $B f$ in Gauss and $v_{\text {eq }}$ in $\mathrm{km} \mathrm{s}^{-1}$. We can insert this relation into Equation (4) to achieve a very rough estimate of the ratio between rotational broadening and Zeeman splitting in sun-like stars. The resulting ratio is

$$
\frac{\Delta v_{\text {Zeeman }}}{v_{\text {eq }}} \approx 0.07 \lambda_{0} g
$$

with $\lambda_{0}$ in $\mu \mathrm{m}$. Thus, at optical wavelengths, the approximate Zeeman shift according to the rotation-magnetic field relation in the non-saturated dynamo regime is usually well below $10 \%$ of rotational broadening. Given the limitations and systematic uncertainties of detailed spectral synthesis, this is a very challenging problem for Zeeman observations. So far, conclusive investigations of Zeeman splitting at infrared wavelengths are lacking, but there is certainly a great need to verify the rotation-magnetic field relation at longer wavelengths $\lambda_{0}$. 


\section{Equipartition}

In stellar atmospheres, a magnetic field is a source of magnetic pressure, $P=B^{2} / 8 \pi$. In hydrostatic equilibrium, this pressure must be balanced by the gas pressure so that magnetic flux is limited by the total gas pressure. Such stability considerations lead to the expectation that stellar magnetic fields in general are limited by atmospheric structure (e.g., Spruit and Zweibel, 1979). Saar and Linsky (1985) and Saar (1990) estimate scaling relations of equipartition magnetic fields in field stars, and Johns-Krull (2007) provides a detailed comparison between magnetic fields in pre-main sequence stars and corresponding equipartition field strengths.

The values of equipartition fields and, therefore, the expected maximum field strengths in main sequence stars are obviously a function of surface temperature and gravity. They turn out to be of the order of $1-2 \mathrm{kG}$ for spectral type $\mathrm{G}, 2-3 \mathrm{kG}$ for spectral type $\mathrm{K}$, and $3.5-4.0 \mathrm{kG}$ for spectral type early- to mid-M (Saar, 1990). From a first glance, this is in remarkable agreement with the maximum average field strengths observed in $\mathrm{M}$ dwarfs. However, the average field of a star with flux tubes satisfying equipartition would probably be much lower than the maximum field because the gas pressure available to balance magnetic pressure must be available in some non-magnetic regions (in other words, $f$ cannot be 1 ).

As discussed above, we have no conclusive information about maximum field strengths in hotter, sun-like stars. We can, therefore, not exclude that maximum field strengths in sun-like stars are significantly lower than $\sim 4 \mathrm{kG}$. Even if the local field strengths in M dwarfs are probably above our estimate of equipartition field strengths, there still may be a scaling of maximum field strengths with gas pressure, and only the absolute value of the limiting field differs somewhat from our approximations. Again, infrared observations of Zeeman splitting are required in active sun-like stars to shed light on this fundamental question.

Equipartition field strengths are predicted to be much lower than $4 \mathrm{kG}$ in pre-main sequence stars. Johns-Krull (2007) has found a significant mismatch between observed field strengths and predictions from equipartition. Average magnetic fields in pre-main sequence stars appear to have typical values of $2.5 \mathrm{kG}$, but predicted field strengths for the young stars of the sample in JohnsKrull (2007) are between 0.5 and $3 \mathrm{kG}$, and the observed field values do not show a correlation with equipartition estimates. Therefore, at least in pre-main sequence stars, magnetic fields exist with magnetic pressure dominating gas pressure. Such stars probably have no field-free regions on their surface. Johns-Krull (2007) also provide evidence that fields in these young stars may be of fossil origin and not generated by a dynamo, although Chabrier and Küker (2006) estimate survival times for fossil fields in fully convective stars well below $1000 \mathrm{yr}$. Assuming that such fields survive much longer, a tentative conclusion consistent with observations is that dynamo generated fields are in agreement with pressure balance, while fossil fields can exceed this boundary. An alternative conclusion from the available data, however, is that the limit for stellar average fields is simply on the order of $3-4 \mathrm{kG}$ in all stars, and pressure balance has a minor effect on the generated field strength. A physical motivation for such a minimalistic approach, however, is missing. 


\section{Geometries of Stellar Magnetic Fields}

Stellar magnetic fields are vector fields. The total strength and energy contained in a stellar magnetic field are probably characteristic of the overall dynamo efficiency and resulting activity, and probably determine the rules of magnetic braking. The geometry of stellar fields adds information that is crucial for our understanding of these effects. The difficulties measuring both, total field strength and geometry, were discussed in the sections above.

As a starting point, again, we can take a look at the Sun. The surface-averaged flux density on the Sun is much lower than on many other stars, but we have a better view on it. Figure 22 show a recent visualization of the Sun's magnetic field during the eruption happening on August 1, 2010. As in stellar magnetic field reconstructions, this visualization rests on model assumptions and leaves some room for fields not captured by the applied methods. Nevertheless, the picture is tremendously rich in details revealing an enormously complex structure of the solar magnetic field.

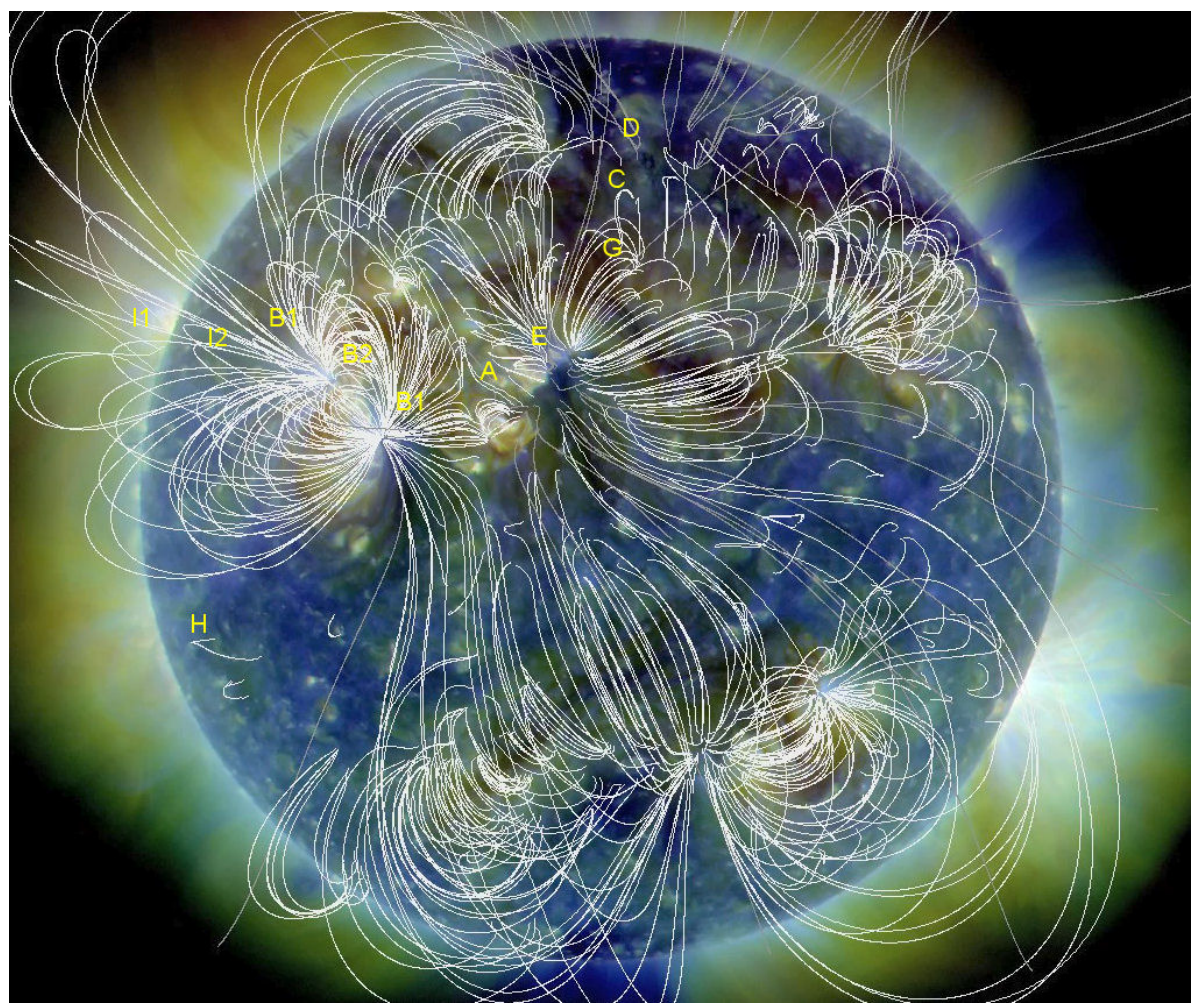

Figure 22: Three-color composite of EUV images of the Sun obtained by the Solar Dynamics Observatory during the Great Eruption of August 1, 2010. White lines show a model of the Sun's complex magnetic field based on an extrapolation for the full-sphere magnetic field (from Schrijver and Title, 2011).

A remarkable feature of the Sun's magnetic field are the large magnetic loops visible in Figure 22. Such loops also occur in images of the solar upper atmosphere where the plasma seems to follow the magnetic field. If the Sun was observed as a star, what part of its magnetic field would we be able to see? It was mentioned before that the Sun's magnetic field would probably be too small to be detected at any rate. In integrated light, Zeeman broadening would be too small by one or two orders of magnitude to produce any detectable signal. Linear polarization would probably remain undetectable, too. In circular polarization, the situation is more difficult. Measurements of polarization from net magnetic fields on the order of one or even a tenth of a Gauss were reported

Living Reviews in Solar Physics

http://www. livingreviews.org/lrsp-2012-1 
for some stars, and this may be within the range of an observable net field of the Sun at a given moment. However, the information about field geometry from such a measurement alone would certainly be very limited.

More information is available if a Doppler Image from a star with a stronger field can be obtained. Such an image takes into account all the net field snapshots visible at different rotational phases, which greatly enhances the detectability of tangled fields. An overview about the current picture of magnetic field geometries in low-mass stars, in particular among stars of spectral type M, was given by Donati and Landstreet (2009). The powerful methods of Least Squares Deconvolution and Zeeman Doppler Imaging have provided a wealth of Doppler Images showing very different pictures of stellar magnetic field geometries. A particularly interesting example are low-mass stars of spectral class M; not only are there many Doppler Images of M stars, this spectral range is also of particular interest for our understanding of the solar and stellar dynamos as pointed out earlier in this review.

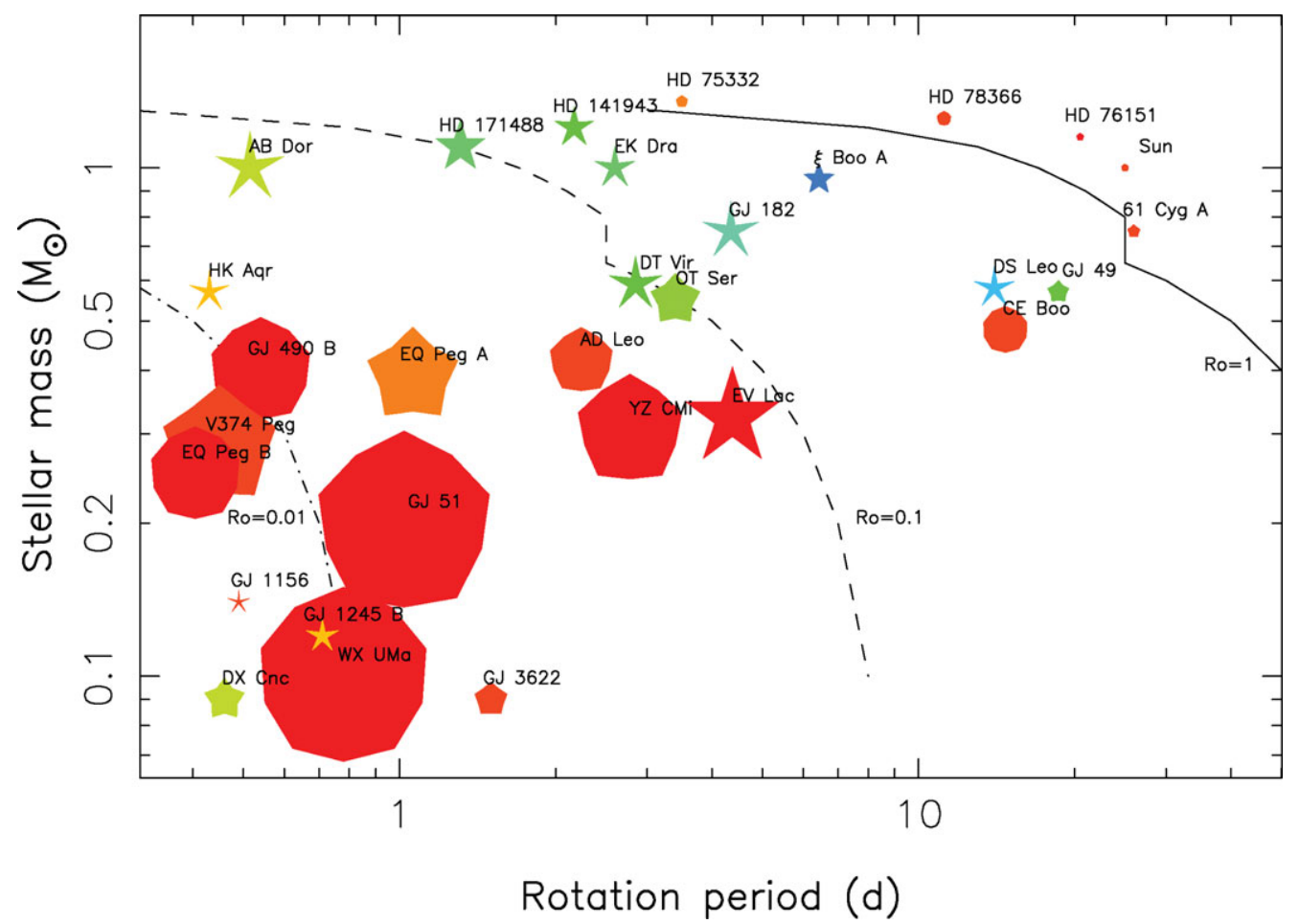

Figure 23: Properties of the large-scale magnetic geometries of cool stars (Donati, 2011) as a function of rotation period and stellar mass. Symbol size indicates magnetic densities with the smallest symbols corresponding to mean large-scale field strengths of $3 \mathrm{G}$ and the largest symbols to $1.5 \mathrm{kG}$. Symbol shapes depict different degrees of axisymmetry of the reconstructed magnetic field (from decagons for purely axisymmetric fields to sharp stars for purely non-axisymmetric fields). Colors illustrate field configuration (dark blue for purely toroidal fields, dark red for purely poloidal fields, intermediate colors for intermediate configurations). Full, dashed, and dash-dot lines trace lines of equal Rossby number $R o=1,0.1$, and 0.01, respectively (from Donati, 2011, reproduced by permission of Cambridge University Press).

Morin et al. (2010) summarized the results from Zeeman Doppler Imaging currently available in M-type stars. Including the results of Morin et al. (2010), Figure 23 shows properties of the large-scale magnetic geometries of cool stars from Donati (2011) in a visualization of magnetic field geometries as a function of mass and rotation period. Many of the stars follow the trend of 
stronger average fields in less massive and more rapidly rotating stars (Donati and Landstreet, 2009). These more active stars have field geometries that seem to be more axisymmetric and predominantly poloidal. This leads to the suggestion that rapidly rotating low-mass stars tend to produce strong, axisymmetric, and poloidal fields. Whether the reason for such a trend is due to rotation, mass (radius), or structural differences in the interior of the stars, is unknown. In any case, a more axisymmetric and poloidal field geometry is not what one expects from the general picture of magnetic dynamos; distributed dynamos in fully convective stars should not be able to produce strong fields that are more symmetric and poloidal than fields in sun-like stars in which the dynamo operating at the tachocline is believed to produce a rather organized global field. The trend towards stronger and more organized fields in low-mass stars is challenged by a number of very-low mass $\left(M \sim 0.1 M_{\odot}\right)$ rapid rotators $(P \sim 1 \mathrm{~d})$ exhibiting rather weak fields and geometries with a low degree of axisymmetry: a number of very-low-mass stars produce fields with entirely different geometries and field strengths (lower left in Figure 23).

It is well known that early-M dwarfs (M0-M3) in the field are generally much less active and slower-rotating than later, fully convective M stars (e.g., Delfosse et al., 1998; Reiners and Basri, 2008; Reiners et al., 2012). Early-M dwarfs appear to suffer much more severe rotational braking so that their activity lifetime is shorter than in later M dwarfs (West et al., 2008), and this can be explained by the severe change in radius and its consequences to angular momentum loss (Reiners and Mohanty, 2011). Do magnetic fields suffer significant change around spectral type M3/M4? In total field strength, visible to Stokes I, no change is detected; differences between field strengths are consistent with the assumption that flux generation is ruled by Rossby number (or rotation period) on both sides of the threshold to fully convective stars. On the other hand, Doppler Images show that differences between sun-like and early-M type stars on one side and very-low-mass stars on the other are enormous. If we assume that these differences are real, low-mass stars must be able to somehow generate fields of structure radically different from fields in sun-like stars. This would probably imply either a small scale dynamo mechanism capable of generating fields with very different global properties, or the co-existence of different dynamo mechanisms in fully convective stars.

It is important to realize that at spectral type M3/M4, severe changes happen also in more basic parameters of these stars, and the reason for a change for example in braking timescales seems to be much more fundamental than magnetic field geometry. For example, from spectral type M2 to M5, radius and mass diminish by more than a factor of two, which is enough to cause the observed differences in rotation and activity (Reiners and Mohanty, 2011). In other words, less effective magnetic braking in fully convective stars does not require a change in field geometry. To what extent such changes may also influence the detectability of magnetic fields, in particular of small-scale magnetic structures, is a question that is important for our understanding of stellar dynamos and, in particular, for the differences between dynamos in fully and partially convective stars.

We have seen in Section 2 that the fraction of the magnetic flux detected in the currently available Zeeman Doppler Images (from Stokes V) may be substantially lower than one, due to cancellation effects or the weak-field approximation. This fraction can be determined if the field is also visible in Stokes I, where the full field is measurable. The typical average field strength of a few hundred Gauss, as detected in Doppler Images, is much lower than average field strengths of magnetically active stars observed in Stokes I that are a typically few kG. We can compare the field measurements in Stokes I and V for stars contained in Tables 2 and 5. This comparison is shown in Figure 24, which is an update of Figure 2 in Reiners and Basri (2009).

Figure 24 shows the average magnetic fields from Stokes I and V, their ratios, and the ratios of magnetic energies as a function of Rossby number and stellar mass. In the top panel, the measurements are shown directly, the center panel shows the ratio between the average magnetic fields $\left\langle B_{V}\right\rangle /\left\langle B_{I}\right\rangle$. For the majority of stars, the ratio is on the order of ten percent or less, which

Living Reviews in Solar Physics

http://www. livingreviews.org/lrsp-2012-1 


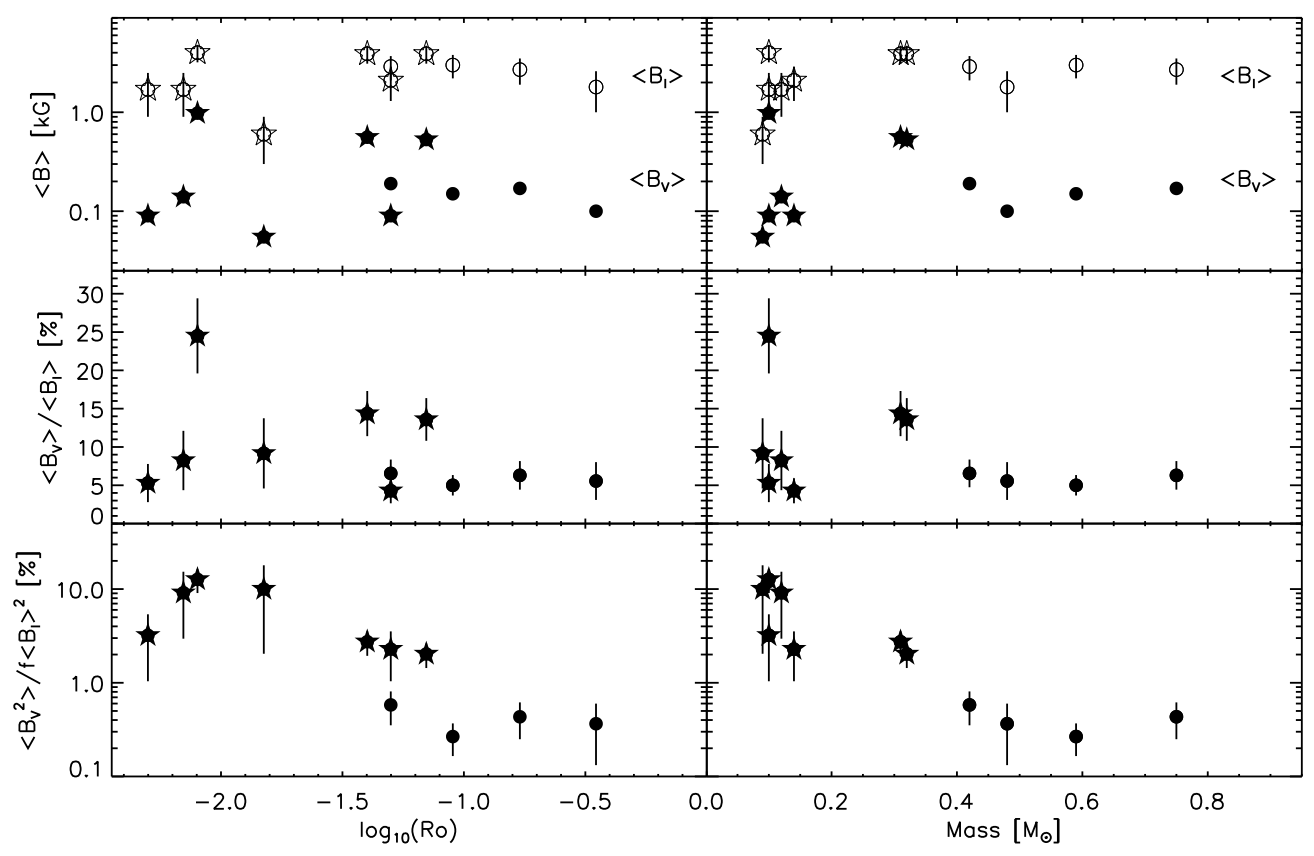

Figure 24: Measurements of $M$ dwarf magnetic fields from Stokes I and Stokes V. Top panel: Average magnetic field - Open symbols: measurements from Stokes I; Filled symbols: measurements from Stokes V. Center panel: Ratio between Stokes V and Stokes I measurements. Bottom panel: Ratio between magnetic energies detected in Stokes V and Stokes I. Circles show objects more massive than $0.4 M_{\odot}$, stars show objects less massive than that.

means that $<10 \%$ of the full magnetic field is detected in the Stokes V map. In other words, more than $90 \%$ of the field detected in Stokes I is invisible to this method. As discussed above, this is probably a consequence of cancellation between field components of different polarity. One very interesting case with a very high value of $\left\langle B_{V}\right\rangle /\left\langle B_{I}\right\rangle$ is the M6 star WX Uma, which has an average field of approximately $1 \mathrm{kG}$ in Stokes V (Gl 51 shows an even higher field but has not yet been investigated with the Stokes I method).

A second observable that comes with the Stokes V maps is average squared magnetic field, $\left\langle B^{2}\right\rangle$, which is proportional to the magnetic energy of the star. Under some basic assumptions, this value can be approximated from the Stokes I measurement, too (see Reiners and Basri, 2009). The ratio between approximate magnetic energies detected in Stokes V and I is shown in the bottom panel of Figure 24, it is between 0.3 and $15 \%$ for the stars considered. In contrast to the conclusions suggested in Donati et al. (2008b) and Reiners and Basri (2009), evidence for a change in magnetic geometries at the boundary between partial and complete convection is not very obvious when the latest results are included. Four of the late-M dwarfs have ratios $\left\langle B_{V}\right\rangle /\left\langle B_{I}\right\rangle$ below $10 \%$ while earlier results suggested that more flux is detectably in Stokes $\mathrm{V}$ in fully convective stars. On the other hand, the ratio of detectable magnetic energies stays rather high in this regime $(\geq 2 \%)$, which may reflect an influence of the convective nature of the star. An important question, however, is why the five low-mass stars with $<0.2 M_{\odot}$ show a relatively high fraction of detected magnetic energy, while they show such a low fraction of detected field strength? This may well be an effect of different magnetic geometries but cannot be clearly identified at this point.

In a typical Zeeman Doppler Image of a low-mass star, about $90 \%$ of the magnetic field and much more than $90 \%$ of the magnetic energy remains undetected. It is a challenging task to derive 
global properties for a field of which only a small fraction is visible. Small-scaled structures like sunspots are in principle difficult to detect in Stokes V measurements, but it is believed that the method can well reproduce the large-scale structures. Nevertheless, in field geometries as complex as shown in Figure 22, it is not immediately clear which part can be reconstructed by a given observation, and which part cannot. Our understanding of magnetic field geometries and magnetic dynamos, both in the Sun and other stars, will therefore depend on whether it will be possible to characterize the properties of the remaining $90 \%$ magnetic flux on stars other than the Sun. 


\section{Beyond Rotation}

Most of our knowledge about the occurrence and strength of magnetic fields in cool stars can be explained by a close relation between rotation and magnetic field generation. While geometries are more difficult to interpret - partly because the observational picture is not unambiguous - the general idea of the rotation-induced dynamo seems to be valid over a rather large scale of physical objects with outer convection zones. There are a few topics beyond this relation between rotation and field generation that should be discussed briefly in this section.

\subsection{A scaling law for saturated planetary and stellar dynamos}

The surface magnetic field of a star is controlled by its rotation rate, but magnetism saturates when rotation is faster than a critical velocity, perhaps associated to the rate where $R o \approx 0.1$. The value of the field strength at this saturation level, however, may vary between objects and depend on additional parameters. Christensen et al. (2009) suggested a scaling law based on energy-flux consistent with the maximum magnetic fields found in rapidly rotating low-mass stars, and some planetary fields like Earth and Jupiter (Figure 25). In this picture, the available heat flux in the convection zone is converted into magnetic energy. A few assumptions are necessary to explain fields of other planets, but the general idea is that a single scaling relation may hold in objects of vastly different dimensions like planets, brown dwarfs, and stars (for a deeper discussion of flux scaling relations, see Christensen, 2010).

In order to test a relation like this one, magnetic fields must be measured in stars in the saturated dynamo regime, i.e., at fast rotation. So far, this was possible only in pre-MS stars and M dwarfs, and fields on the order of a few $\mathrm{kG}$ were found here. The two species have available a comparable amount of heat flux so that the scaling relation yields comparable results for both groups, which is consistent with the empirical results from the field measurements. In order to further test the applicability of the scaling relation, it would be necessary to observe the saturation level in stars of very different nature, brown dwarfs, and finally exoplanets. The scaling law provides a prediction for magnetic fields in brown dwarfs; according to the relation, magnetic fields on the order of several hundred Gauss up to kG-strength should exist on rapidly rotating, evolved brown dwarfs. 


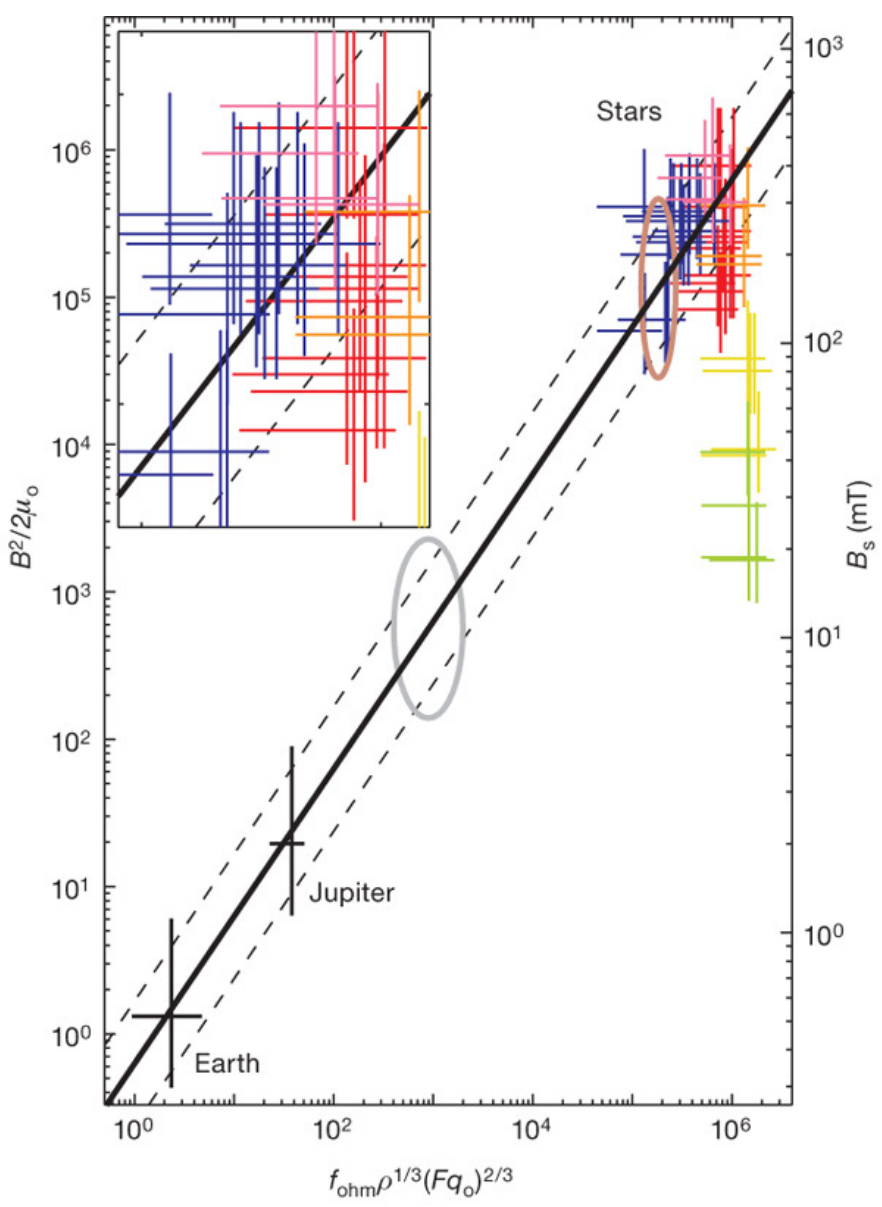

Figure 25: Magnetic energy density in the dynamo vs. a function of density and bolometric flux (both in units of $\mathrm{J} \mathrm{m}^{-3}$ ) according to Christensen et al. (2009). The scale on the right shows r.m.s. field strength at the dynamo surface. Blue: T Tauri stars; red: old $\mathrm{M}$ dwarfs. Black lines show the rescaled fit from Christensen et al. (2009) with $3 \sigma$ uncertainties (solid and dashed lines, respectively). The stellar field is enlarged in the inset. Brown and grey ellipses indicate predicted locations of a brown dwarf with $1500 \mathrm{~K}$ surface temperature and an extrasolar planet with seven Jupiter masses, respectively (from Christensen et al., 2009). 


\subsection{Brown dwarfs}

The sample of objects investigated for magnetic fields by Reiners et al. (2009b) and Reiners and Basri (2010) includes a number of brown dwarfs. All of them are of spectral type late-M implying that they are young, a few of them probably only ten Myr or less. So far, no significant magnetic field could be detected in a brown dwarf using Zeeman splitting although radio observations in brown dwarfs provide evidence for kG-strength magnetic fields (see Section 2.3). Interestingly, all young brown dwarfs investigated for Zeeman splitting so far are known accretors. Thus, they harbor a disk, which is probably the reason why they are not rotating beyond the critical rate at which Zeeman broadening becomes undetectable. Upper limits for the magnetic fields in young, accreting brown dwarfs are on the order of a few hundred Gauss, significantly lower than fields found in higher-mass young $\mathrm{T}$ Tauri stars or in older stars of the same spectral type. The field limits are also significantly below the predictions from the scaling model introduced in Section 7.1. It is currently unknown whether the low fields in accreting brown dwarfs are due to a less effective dynamo, the influence of accretion, or some other effect.

More evolved brown dwarfs at ages higher than typical disk lifetimes of ten Myr are usually rotating too fast for a successful detection of Zeeman broadening, and they are often also too faint for current spectroscopic instrumentation. An observation of a magnetic field (or its upper limit) in non-accreting brown dwarfs, regardless of age, would be important to make progress in this field.

\subsection{Fossil or dynamo fields in young low-mass stars}

One remarkable result from the observation of magnetic fields in young low-mass stars is the relatively small scatter in the fields detected in Stokes I. Most of the fields reported for 14 classical T Tauri stars investigated by Johns-Krull (2007) have average flux densities on the order of $2 \mathrm{kG}$ and do not follow predictions according to equipartition. This sample of stars was augmented with 14 other T Tauri stars by Yang and Johns-Krull (2011) still finding similar results. A potential explanation put forward by Yang and Johns-Krull (2011) is that the field strength in these young stars is not actually maintained through a magnetic dynamo that would be affected by the scatter of physical properties in the rather diverse sample of young stars. Instead, Yang and Johns-Krull (2011) propose that the weak scatter in magnetic field measurements is the result of the gradually weakening magnetic flux from a fossil field. Because the stars are still contracting, the younger stars with more magnetic flux, $4 \pi R_{\star}^{2} B$, are the stars that are larger than the older stars with less magnetic flux. According to pre-MS evolutionary models, the observed average field strengths are consistent with magnetic flux decreasing by about a factor of ten between 1 and 10 Myr according to the evolution of available convective energy. However, estimates of survival times of fossil fields predict much faster decay well below $1000 \mathrm{yr}$ (Chabrier and Küker, 2006). Furthermore, the weakening of total flux with age shown by Yang and Johns-Krull (2011) is based on age estimates derived from comparison to evolutionary models. However, Baraffe et al. (2009) show that episodic accretion can lead to a luminosity spread that can be misinterpreted as an age spread on the order of $10 \mathrm{Myr}$, hence age and radius estimates have large uncertainties in this regime. More Stokes I observations of average magnetic fields in stars of several $10 \mathrm{Myr}$ age would be helpful to see whether the total flux is indeed weakening on this timescale.

The obvious alternative to fossil fields are dynamo generated fields in young low-mass stars, and convective timescales are supporting this scenario (see above). Furthermore, Zeeman Doppler maps suggest that large-scale fields in classical $\mathrm{T}$ Tauri stars can undergo secular variations on timescales of a few years (e.g., Donati and Landstreet, 2009; Donati et al., 2011a). If the variability in these magnetic field maps is indeed due to a change in magnetic geometry of the star, this readily implies that fields cannot be of fossil origin but must be generated by a dynamo mechanism.

Nevertheless, an idea similar to the decay of fossil magnetic flux may be useful to answer the question why young (accreting) brown dwarfs have such small fields. Effective dynamos may not 
yet be operating in these young objects. The radii of young brown dwarfs are much larger than radii in older brown dwarfs and low-mass field stars, implying that average fields may be very low if the available magnetic flux (not a generated field) is the limiting factor. Observational evidence for any of these scenarios among brown dwarfs, however, demands conclusive magnetic field observations.

\section{Summary}

Cool-star magnetic fields are in the center of interest for a large number of reasons. Their influence on star-formation, solar and stellar activity, habitability of planets, magnetic dynamos, stellar structure, angular momentum evolution, and many other topics, put magnetic fields in the focus of vastly different research areas. Our vision of magnetic fields in different astronomical contexts, however, is motivated by only a very limited number of empirical facts, but influenced by an almost unlimited number of assumptions. An arsenal of different methods is available to search for magnetic fields in cool stars, but usually the different methods provide insights into very different aspects of magnetism.

Together with observations of stellar activity and rotation, direct magnetic fields measurements and reconstruction of field geometries provide an empirical picture of stellar dynamos. With observations of young stars on one side, and detailed information about the solar magnetic field on the other, we have boundary conditions that allow an investigation of an evolution of stellar magnetism. Our observations are crossing the physically important boundaries between partially and fully convective stars, between saturated activity and unsaturated stars, and between stars and brown dwarfs.

Magnetic fields in cool stars are just on the edge of detectability for our methods. The interpretation of results from the various methods opens a parameter space that certainly contains deep information about the fields, their geometry, and the underlying physical principles. Unfortunately, it is not always clear how to interpret our observational results, and how measurements from different analyses can be compared. Therefore, it is not only important to improve the general observational picture by collecting more observational material, but it is perhaps of even greater importance to understand the assumptions in and limitations of the methodology we are applying. Technology and computer power is improving rapidly and will at some point make more and more details available to direct observation. Nevertheless, the design of future instrumentation is driven by expectations and interpretation of earlier results. Given the fact that still a large fraction of the magnetic universe is unknown, we should be prepared to find a richness of magnetic phenomena that does not yet exist in our imagination of stellar magnetic fields - and we must ensure that our observations can find it.

\section{Acknowledgments}

I am very thankful to Steve Saar for his careful reading and thoughtful comments on the manuscript, and to Karel Schrijver and anomymous referees for very useful comments and their help to shape this article. Financial support is acknowledged from the Deutsche Forschungsgemeinschaft through the Emmy Noether programme, RE 1664/4-1, and the Heisenberg Programme, RE 1664/9-1. 


\section{References}

Afram, N., Reiners, A. and Berdyugina, S.V., 2009, "Magnetic Fields on M Dwarfs Measured with FeH", in Solar Polarization 5: In Honor of Jan Olof Stenflo, Centro Stefano Franscini-Monte Veritá, Ascona, Switzerland, 17-21 September, 2007, (Eds.) Berdyugina, S.V., Nagendra, K.N., Ramelli, R., vol. 405 of ASP Conference Series, p. 527, Astronomical Society of the Pacific, San Francisco. [ADS] (Cited on page 32.)

Al-Malki, M.B., Simmons, J.F.L., Ignace, R., Brown, J.C. and Clarke, D., 1999, "Scattering polarization due to light source anisotropy. I. Large spherical envelope", Astron. Astrophys., 347, 919-926. [ADS] (Cited on page 23.)

Alekseev, I.Y., 2003, "Broad-band Multicolor Photometry and Polarimetry of Spotted Stars", Astron. Rep., 47, 430-442. [DOI], [ADS] (Cited on page 23.)

Anderson, R.I., Reiners, A. and Solanki, S.K., 2010, "On detectability of Zeeman broadening in optical spectra of F- and G-dwarfs", Astron. Astrophys., 522, A81. [DOI], [ADS], [arXiv:1008.2213 [astroph.SR]] (Cited on pages 29, 30, 31, and 36.)

Aurière, M., Wade, G.A., Konstantinova-Antova, R., Charbonnel, C., Catala, C., Weiss, W.W., Roudier, T., Petit, P., Donati, J.-F., Alecian, E., Cabanac, R., van Eck, S., Folsom, C.P. and Power, J., 2009, "Discovery of a weak magnetic field in the photosphere of the single giant Pollux", Astron. Astrophys., 504, 231-237. [DOI], [ADS], [arXiv:0907.1423 [astro-ph.SR]] (Cited on page 44.)

Aurière, M., Donati, J.-F., Konstantinova-Antova, R., Perrin, G., Petit, P. and Roudier, T., 2010, "The magnetic field of Betelgeuse: a local dynamo from giant convection cells?", Astron. Astrophys., 516, L2. [DOI], [ADS], [arXiv:1005.4845 [astro-ph.SR]] (Cited on page 44.)

Bagnulo, S., Szeifert, T., Wade, G.A., Landstreet, J.D. and Mathys, G., 2002, "Measuring magnetic fields of early-type stars with FORS1 at the VLT", Astron. Astrophys., 389, 191-201. [DOI], [ADS] (Cited on pages 23 and 24.)

Baliunas, S.L., Donahue, R.A., Soon, W.H., Horne, J.H., Frazer, J., Woodard-Eklund, L., Bradford, M., Rao, L.M., Wilson, O.C., Zhang, Q., Bennett, W., Briggs, J., Carroll, S.M., Duncan, D.K., Figueroa, D., Lanning, H.H., Misch, T., Mueller, J., Noyes, R.W., Poppe, D., Porter, A.C., Robinson, C.R., Russell, J., Shelton, J.C., Soyumer, T., Vaughan, A.H. and Whitney, J.H., 1995, "Chromospheric variations in main-sequence stars. II.", Astrophys. J., 438, 269-287. [DOI], [ADS] (Cited on page 25.)

Baraffe, I., Chabrier, G. and Gallardo, J., 2009, "Episodic Accretion at Early Stages of Evolution of LowMass Stars and Brown Dwarfs: A Solution for the Observed Luminosity Spread in H-R Diagrams?", Astrophys. J. Lett., 702, L27-L31. [DOI], [ADS], [arXiv:0907.3886 [astro-ph.SR]] (Cited on page 59.)

Barnes, S.A. and Kim, Y.-C., 2010, "Angular Momentum Loss from Cool Stars: An Empirical Expression and Connection to Stellar Activity", Astrophys. J., 721, 675-685. [DOI], [ADS], [arXiv:1104.2350 [astroph.SR]] (Cited on page 45.)

Basri, G., 1986, "Rossby or Not Rossby", in Cool Stars, Stellar Systems and the Sun: Fourth Cambridge Workshop, Santa Fe, New Mexico, 16-18 October, 1985, (Eds.) Zeilik, M., Gibson, D.M., vol. 254 of Lecture Notes in Physics, p. 184, Springer, Berlin; New York. [ADS] (Cited on page 45.)

Basri, G. and Marcy, G.W., 1988, "Physical realism in the analysis of stellar magnetic fields", Astrophys. J., 330, 274-285. [DOI], [ADS] (Cited on page 36.)

Basri, G., Valenti, J.A. and Marcy, G.W., 1990, "Physical realism in the analysis of stellar magnetic fields. III. Flux tubes and multicomponent atmospheres", Astrophys. J., 360, 650-661. [DOI], [ADS] (Cited on page 28.)

Basri, G., Marcy, G.W. and Valenti, J.A., 1992, "Limits on the magnetic flux of pre-main-sequence stars", Astrophys. J., 390, 622-633. [DOI], [ADS] (Cited on pages 17, 34, and 39.) 
Beckers, J.M., 1969, A table of Zeeman Multiplets, Physical Science Research Papers no. 371, SPO and Air Force Cambridge Research Laboratories, Bedford, MA. [ADS] (Cited on pages 9 and 10.)

Benz, A.O. and Güdel, M., 1994, "The soft X-ray/microwave ratio of flares and coronae", Astron. Astrophys., 285, 621-630. [ADS] (Cited on page 26.)

Berdyugina, S.V., 2005, "Starspots: A Key to the Stellar Dynamo", Living Rev. Solar Phys., 2, lrsp-20058. [ADS]. URL (accessed 21 June 2011): http://www.livingreviews.org/lrsp-2005-8 (Cited on page 9.)

Berdyugina, S.V. and Solanki, S.K., 2002, "The molecular Zeeman effect and diagnostics of solar and stellar magnetic fields. I. Theoretical spectral patterns in the Zeeman regime", Astron. Astrophys., $\mathbf{3 8 5}$, 701-715. [DOI], [ADS] (Cited on page 32.)

Berger, E., Rutledge, R.E., Reid, I.N., Bildsten, L., Gizis, J.E., Liebert, J., Martín, E., Basri, G., Jayawardhana, R., Brandeker, A., Fleming, T.A., Johns-Krull, C.M., Giampapa, M.S., Hawley, S.L. and Schmitt, J.H.M.M., 2005, "The Magnetic Properties of an L Dwarf Derived from Simultaneous Radio, X-Ray, and H $\alpha$ Observations", Astrophys. J., 627, 960-973. [DOI], [ADS], [arXiv:astro-ph/0502384] (Cited on page 26.)

Berger, E., Rutledge, R.E., Phan-Bao, N., Basri, G., Giampapa, M.S., Gizis, J.E., Liebert, J., Martín, E. and Fleming, T.A., 2009, "Periodic Radio and $\mathrm{H} \alpha$ Emission from the L Dwarf Binary 2MASSW J0746425+200032: Exploring the Magnetic Field Topology and Radius Of An L Dwarf", Astrophys. J., 695, 310-316. [DOI], [ADS], [arXiv:0809.0001] (Cited on page 35.)

Blake, C.H., Charbonneau, D. and White, R.J., 2010, "The NIRSPEC Ultracool Dwarf Radial Velocity Survey", Astrophys. J., 723, 684-706. [DOI], [ADS], [arXiv:1008.3874 [astro-ph.SR]] (Cited on page 48.)

Bopp, B.W., Saar, S.H., Ambruster, C., Feldman, P., Dempsey, R.C., Allen, M. and Barden, S.C., 1989, "The active chromosphere binary HD 17433 (VY Arietis)", Astrophys. J., 339, 1059-1072. [DOI], [ADS] (Cited on page 40.)

Borra, E.F., Edwards, G. and Mayor, M., 1984, "The magnetic fields of the late-type stars", Astrophys. J., 284, 211-222. [DOI], [ADS] (Cited on page 41.)

Bouvier, J., Alencar, S.H.P., Harries, T.J., Johns-Krull, C.M. and Romanova, M.M., 2007, "Magnetospheric Accretion in Classical T Tauri Stars", in Protostars and Planets V, (Eds.) Reipurth, B., Jewitt, D., Keil, K., pp. 479-494, University of Arizona Press, Tucson, AZ. [ADS], [arXiv:astro-ph/0603498] (Cited on page 33.)

Brown, D.N. and Landstreet, J.D., 1981, "A search for weak longitudinal magnetic fields on late-type stars", Astrophys. J., 246, 899-904. [DOI], [ADS] (Cited on page 41.)

Bruning, D.H., Marcy, G.W. and Chenoweth Jr, R.E., 1987, "Magnetic fields on K and M dwarfs", in Cool Stars, Stellar Systems and the Sun: Fifth Cambridge Workshop, Boulder, Colorado, July 7-11, 1987, (Eds.) Linsky, J.L., Stencel, E.E., vol. 291 of Lecture Notes in Physics, pp. 36-37, Springer, Berlin; New York. [DOI], [ADS] (Cited on page 40.)

Carroll, T.A., Kopf, M., Ilyin, I. and Strassmeier, K.G., 2007, "Zeeman-Doppler imaging of late-type stars: The surface magnetic field of II Peg", Astron. Nachr., 328, 1043. [DOI], [ADS], [arXiv:0712.3769] (Cited on page 43.)

Catala, C., Donati, J.-F., Shkolnik, E., Bohlender, D. and Alecian, E., 2007, "The magnetic field of the planet-hosting star $\tau$ Bootis", Mon. Not. R. Astron. Soc., 374, L42-L46. [DOI], [ADS], [arXiv:astroph/0610758] (Cited on page 43.)

Chabrier, G. and Küker, M., 2006, "Large-scale $\alpha^{2}$-dynamo in low-mass stars and brown dwarfs", Astron. Astrophys., 446, 1027-1037. [DOI], [ADS], [arXiv:astro-ph/0510075] (Cited on pages 51 and 59.) 
Charbonneau, P., 2010, "Dynamo Models of the Solar Cycle", Living Rev. Solar Phys., 7, lrsp-2010-3. [ADS]. URL (accessed 21 June 2011):

http://www.livingreviews.org/lrsp-2010-3 (Cited on page 45.)

Christensen, U.R., 2010, "Dynamo Scaling Laws and Applications to the Planets", Space Sci. Rev., 152, 565-590. [DOI], [ADS] (Cited on page 57.)

Christensen, U.R., Holzwarth, V. and Reiners, A., 2009, "Energy flux determines magnetic field strength of planets and stars", Nature, 457, 167-169. [DOI], [ADS] (Cited on pages 57 and 58.)

Condon, E.U. and Shortley, G.H., 1963, The Theory of Atomic Spectra, Cambridge University Press, Cambridge; New York. [ADS], [Google Books] (Cited on page 9.)

Daou, A.G., Johns-Krull, C.M. and Valenti, J.A., 2006, "Spectropolarimetry of the Classical T Tauri Star T Tauri", Astron. J., 131, 520-526. [DOI], [ADS] (Cited on pages 43 and 44.)

Delfosse, X., Forveille, T., Perrier, C. and Mayor, M., 1998, "Rotation and chromospheric activity in field M dwarfs", Astron. Astrophys., 331, 581-595. [ADS] (Cited on page 54.)

Deutsch, A.J., 1958, "Harmonic analysis of the periodic spectrum variables", in Electromagnetic Phenomena in Cosmical Physics, Stockholm, Sweden, 27-31 August 1956, (Ed.) Lehnert, B., vol. 6 of IAU Symposia, pp. 209-221, Cambridge University Press, Cambridge; New York. [ADS], [Google Books] (Cited on page 18.)

Donahue, R.A., Saar, S.H. and Baliunas, S.L., 1996, "A Relationship between Mean Rotation Period in Lower Main-Sequence Stars and Its Observed Range", Astrophys. J., 466, 384-391. [DOI], [ADS] (Cited on page 50.)

Donati, J.-F., 1999, "Magnetic cycles of HR 1099 and LQ Hydrae", Mon. Not. R. Astron. Soc., 302, 457-481. [DOI], [ADS] (Cited on page 43.)

Donati, J.-F., 2001, "Imaging the Magnetic Topologies of Cool Active Stars", in Astrotomography: Indirect Imaging Methods in Observational Astronomy, (Eds.) Boffin, H.M.J., Steeghs, D., Cuypers, J., vol. 573 of Lecture Notes in Physics, pp. 207-231, Springer, Berlin; New York. [ADS], [Google Books] (Cited on pages 19,20 , and 21.)

Donati, J.-F., 2011, "Large-scale magnetic fields of low-mass dwarfs: the many faces of dynamo", in Astrophysical Dynamics: From Stars to Galaxies, IAU Symposium 271, 21 - 25 June 2010, Nice, France, (Eds.) Brummell, N.H., Brun, A.S., Miesch, M.S., Ponty, Y., vol. 6 of Proc. IAU, pp. 23-31, Cambridge University Press, Cambridge; New York. [DOI], [ADS] (Cited on page 53.)

Donati, J.-F. and Brown, S.F., 1997, "Zeeman-Doppler imaging of active stars. V. Sensitivity of maximum entropy magnetic maps to field orientation", Astron. Astrophys., 326, 1135-1142. [ADS] (Cited on pages 19, 21, and 23.)

Donati, J.-F. and Collier Cameron, A., 1997, "Differential rotation and magnetic polarity patterns on AB Doradus", Mon. Not. R. Astron. Soc., 291, 1-19. [ADS] (Cited on page 23.)

Donati, J.-F. and Landstreet, J.D., 2009, "Magnetic Fields of Nondegenerate Stars", Annu. Rev. Astron. Astrophys., 47, 333-370. [DOI], [ADS], [arXiv:0904.1938 [astro-ph.SR]] (Cited on pages 9, 19, 53, 54, and 59.)

Donati, J.-F., Semel, M., Rees, D.E., Taylor, K. and Robinson, R.D., 1990, "Detection of a magnetic region on HR 1099", Astron. Astrophys., 232, L1-L4. [ADS] (Cited on page 41.)

Donati, J.-F., Semel, M. and Rees, D.E., 1992, "Circularly polarized spectroscopic observations of RS CVn systems", Astron. Astrophys., 265, 669-681. [ADS] (Cited on page 41.) 
Donati, J.-F., Semel, M., Carter, B.D., Rees, D.E. and Collier Cameron, A., 1997, "Spectropolarimetric observations of active stars", Mon. Not. R. Astron. Soc., 291, 658-682. [ADS] (Cited on pages 22 and 41.)

Donati, J.-F., Collier Cameron, A., Hussain, G.A.J. and Semel, M., 1999, "Magnetic topology and prominence patterns on AB Doradus", Mon. Not. R. Astron. Soc., 302, 437-456. [DOI], [ADS] (Cited on page 43.)

Donati, J.-F., Forveille, T., Collier Cameron, A., Barnes, J.R., Delfosse, X., Jardine, M.M. and Valenti, J.A., 2006, "The Large-Scale Axisymmetric Magnetic Topology of a Very-Low-Mass Fully Convective Star", Science, 311, 633-635. [DOI], [ADS], [arXiv:astro-ph/0602069] (Cited on page 43.)

Donati, J.-F., Jardine, M.M., Gregory, S.G., Petit, P., Bouvier, J., Dougados, C., Ménard, F., Collier Cameron, A., Harries, T.J., Jeffers, S.V. and Paletou, F., 2007, "Magnetic fields and accretion flows on the classical T Tauri star V2129 Oph", Mon. Not. R. Astron. Soc., 380, 1297-1312. [DOI], [ADS], [arXiv:0709.1414] (Cited on page 44.)

Donati, J.-F., Jardine, M.M., Gregory, S.G., Petit, P., Paletou, F., Bouvier, J., Dougados, C., Ménard, F., Collier Cameron, A., Harries, T.J., Hussain, G.A.J., Unruh, Y.C., Morin, J., Marsden, S.C., Manset, N., Aurière, M., Catala, C. and Alecian, E., 2008a, "Magnetospheric accretion on the T Tauri star BP Tauri", Mon. Not. R. Astron. Soc., 386, 1234-1251. [DOI], [ADS], [arXiv:0802.2052] (Cited on page 44.)

Donati, J.-F., Morin, J., Petit, P., Delfosse, X., Forveille, T., Aurière, M., Cabanac, R., Dintrans, B., Fares, R., Gastine, T., Jardine, M.M., Lignières, F., Paletou, F., Ramírez Vélez, J.C. and Théado, S., 2008b, "Large-scale magnetic topologies of early M dwarfs", Mon. Not. R. Astron. Soc., 390, 545-560. [DOI], [ADS], [arXiv:0809.0269] (Cited on pages 41, 43, and 55.)

Donati, J.-F., Skelly, M.B., Bouvier, J., Jardine, M.M., Gregory, S.G., Morin, J., Hussain, G.A.J., Dougados, C., Ménard, F. and Unruh, Y.C., 2010, "Complex magnetic topology and strong differential rotation on the low-mass T Tauri star V2247 Oph", Mon. Not. R. Astron. Soc., 402, 1426-1436. [DOI], [ADS], [arXiv:0911.1080 [astro-ph.SR]] (Cited on page 44.)

Donati, J.-F., Bouvier, J., Walter, F.M., Gregory, S.G., Skelly, M.B., Hussain, G.A.J., Flaccomio, E., Argiroffi, C., Grankin, K.N., Jardine, M.M., Ménard, F., Dougados, C. and Romanova, M.M., 2011a, "Non-stationary dynamo and magnetospheric accretion processes of the classical T Tauri star V2129 Oph", Mon. Not. R. Astron. Soc., 412, 2454-2468. [DOI], [ADS], [arXiv:1011.4789 [astro-ph.SR]] (Cited on pages 44 and 59.)

Donati, J.-F., Gregory, S.G., Alencar, S.H.P., Bouvier, J., Hussain, G., Skelly, M.B., Dougados, C., Jardine, M.M., Ménard, F., Romanova, M.M. and Unruh, Y.C. [MaPP collaboration], 2011b, "The large-scale magnetic field and poleward mass accretion of the classical T Tauri star TW Hya", Mon. Not. R. Astron. Soc., 417, 472-487. [DOI], [ADS], [arXiv:1106.4162 [astro-ph.SR]] (Cited on page 44.)

Donati, J.-F., Gregory, S.G., Montmerle, T., Maggio, A., Argiroffi, C., Sacco, G., Hussain, G., Kastner, J., Alencar, S.H.P., Audard, M., Bouvier, J., Damiani, F., Güdel, M., Huenemoerder, D.P. and Wade, G.A., 2011c, "The close classical T Tauri binary V4046 Sgr: complex magnetic fields and distributed mass accretion", Mon. Not. R. Astron. Soc., 417, 1747-1759. [DOI], [ADS] (Cited on page 42.)

Dulk, G.A., 1985, "Radio emission from the sun and stars", Annu. Rev. Astron. Astrophys., 23, 169-224. [DOI], [ADS] (Cited on page 26.)

Dunstone, N.J., Hussain, G.A.J., Collier Cameron, A., Marsden, S.C., Jardine, M.M., Stempels, H.C., Ramírez Vélez, J.C. and Donati, J.-F., 2008, "The first magnetic maps of a pre-main-sequence binary star system - HD155555", Mon. Not. R. Astron. Soc., 387, 481-496. [DOI], [ADS], [arXiv:0803.0837] (Cited on page 44.)

Durney, B.R. and Latour, J., 1978, "On the angular momentum loss of late-type stars", Geophys. Astrophys Fluid Dyn., 9, 241-255. [ADS] (Cited on page 45.) 
Durney, B.R. and Robinson, R.D., 1982, "On an estimate of the dynamo-generated magnetic fields in late-type stars", Astrophys. J., 253, 290-297. [DOI], [ADS] (Cited on page 47.)

Falk, A.E. and Wehlau, W.H., 1974, "Harmonic Analysis of the Line Profiles of an Oblique Rotator", Astrophys. J., 192, 409-416. [DOI], [ADS] (Cited on page 18.)

Gaulme, P., Deheuvels, S., Weiss, W.W., Mosser, B., Moutou, C., Bruntt, H., Donati, J.-F., Vannier, M., Guillot, T., Appourchaux, T., Michel, E., Auvergne, M., Samadi, R., Baudin, F., Catala, C. and Baglin, A., 2010, "HD 46375: seismic and spectropolarimetric analysis of a young Sun hosting a Saturnlike planet", Astron. Astrophys., 524, A47. [DOI], [ADS], [arXiv:1011.2671 [astro-ph.SR]] (Cited on page 43.)

Gilliland, R.L., 1986, "Relation of chromospheric activity to convection, rotation, and pre-main-sequence evolution", Astrophys. J., 300, 339-347. [DOI], [ADS] (Cited on page 45.)

Golub, L., Giampapa, M.S. and Worden, S.P., 1983, "The magnetic field on the RS Canum Venaticorum star Lambda Andromedae", Astrophys. J. Lett., 268, L121-L125. [DOI], [ADS] (Cited on page 40.)

Goncharskii, A.V., Stepanov, V.V., Kokhlova, V.L. and Yagola, A.G., 1977, "Reconstruction of local line profiles from those observed in an Ap spectrum", Sov. Astron. Lett., 3, 147-149. [ADS] (Cited on page 18.)

Gondoin, P., Giampapa, M.S. and Bookbinder, J.A., 1985, "Stellar magnetic field measurements utilizing infrared spectral lines", Astrophys. J., 297, 710-718. [DOI], [ADS] (Cited on page 40.)

Gray, D.F., 1984, "Measurements of Zeeman broadening in F, G, and K dwarfs", Astrophys. J., 277, 640-647. [DOI], [ADS] (Cited on pages 17 and 40.)

Gray, D.F., 1985, "An apparent universal magnetic constant for cool stars", Publ. Astron. Soc. Pac., 97, 719-724. [DOI], [ADS] (Cited on page 28.)

Güdel, M., 2002, "Stellar Radio Astronomy: Probing Stellar Atmospheres from Protostars to Giants", Annu. Rev. Astron. Astrophys., 40, 217-261. [DOI], [ADS], [arXiv:astro-ph/0206436] (Cited on pages 9 and 26.)

Güdel, M., 2004, "X-ray astronomy of stellar coronae", Astron. Astrophys. Rev., 12, 71-237. [DOI], [ADS], [arXiv:astro-ph/0406661] (Cited on pages 9 and 25.)

Guenther, E.W., Lehmann, H., Emerson, J.P. and Staude, J., 1999, "Measurements of magnetic field strength on T Tauri stars", Astron. Astrophys., 341, 768-783. [ADS] (Cited on pages 34 and 39.)

Hall, J.C., 2008, "Stellar Chromospheric Activity", Living Rev. Solar Phys., 5, lrsp-2008-2. [ADS]. URL (accessed 21 June 2011):

http://www.livingreviews.org/lrsp-2008-2 (Cited on pages 9 and 25.)

Hallinan, G., Antonova, A., Doyle, J.G., Bourke, S., Lane, C. and Golden, A., 2008, "Confirmation of the Electron Cyclotron Maser Instability as the Dominant Source of Radio Emission from Very Low Mass Stars and Brown Dwarfs", Astrophys. J., 684, 644-653. [DOI], [ADS], [arXiv:0805.4010] (Cited on pages 26 and 35.)

Hanle, W., 1924, "Über magnetische Beeinflussung der Polarisation der Resonanzfluoreszenz", Z. Phys., 30, 93-105. [DOI] (Cited on page 9.)

Helling, C., Jardine, M.M. and Mokler, F., 2011, "Ionization in Atmospheres of Brown Dwarfs and Extrasolar Planets. II. Dust-induced Collisional Ionization", Astrophys. J., 737, 38. [DOI], [ADS], [arXiv:1105.4409 [astro-ph.SR]] (Cited on page 48.)

Huovelin, J. and Saar, S.H., 1991, "Broad-band linear polarization in cool stars. I. Models and spatial effects for magnetic and scattering regions", Astrophys. J., 374, 319-329. [DOI], [ADS] (Cited on page 23.) 
Hussain, G.A.J., Collier Cameron, A., Jardine, M.M., Dunstone, N.J., Ramírez Vélez, J.C., Stempels, H.C., Donati, J.-F., Semel, M., Aulanier, G., Harries, T.J., Bouvier, J., Dougados, C., Ferreira, J., Carter, B.D. and Lawson, W.A., 2009, "Surface magnetic fields on two accreting T Tauri stars: CV Cha and CR Cha", Mon. Not. R. Astron. Soc., 398, 189-200. [DOI], [ADS], [arXiv:0905.0914 [astro-ph.SR]] (Cited on page 44.)

Johns-Krull, C.M., 2007, "The Magnetic Fields of Classical T Tauri Stars", Astrophys. J., 664, 975-985. [DOI], [ADS], [arXiv:0704.2923] (Cited on pages 34, 39, 42, 51, and 59.)

Johns-Krull, C.M. and Valenti, J.A., 1996, "Detection of Strong Magnetic Fields on M Dwarfs", Astrophys. J. Lett., 459, L95-L98. [DOI], [ADS] (Cited on pages 31, 32, and 37.)

Johns-Krull, C.M. and Valenti, J.A., 2000, "Measurements of stellar magnetic fields", in Stellar Clusters and Associations: Convection, Rotation, and Dynamos, Proceedings for a meeting held in Mondello, Palermo, Italy, 25-28 May, 1999, (Eds.) Pallavicini, R., Micela, G., Sciortino, S., vol. 198 of ASP Conference Series, pp. 371-380, Astronomical Society of the Pacific, San Francisco. [ADS] (Cited on pages $31,32,37$, and 44 .)

Johns-Krull, C.M., Valenti, J.A., Hatzes, A.P. and Kanaan, A., 1999a, "Spectropolarimetry of Magnetospheric Accretion on the Classical T Tauri Star BP Tauri", Astrophys. J. Lett., 510, L41-L44. [DOI], [ADS] (Cited on page 44.)

Johns-Krull, C.M., Valenti, J.A. and Koresko, C., 1999b, "Measuring the Magnetic Field on the Classical T Tauri Star BP Tauri", Astrophys. J., 516, 900-915. [DOI], [ADS] (Cited on pages 33, 34, and 39.)

Johns-Krull, C.M., Valenti, J.A. and Saar, S.H., 2004, "Testing the Reality of Strong Magnetic Fields on T Tauri Stars: The Naked T Tauri Star Hubble 4", Astrophys. J., 617, 1204-1215. [DOI], [ADS], [arXiv:astro-ph/0409268] (Cited on page 39.)

Kim, Y.-C. and Demarque, P., 1996, "The Theoretical Calculation of the Rossby Number and the 'Nonlocal' Convective Overturn Time for Pre-Main-Sequence and Early Post-Main-Sequence Stars", Astrophys. J., 457, 340. [DOI], [ADS] (Cited on page 29.)

Kim, Y.-C., Fox, P.A., Demarque, P. and Sofia, S., 1996, "Modeling Convection in the Outer Layers of the Sun: A Comparison with Predictions of the Mixing-Length Approximation", Astrophys. J., 461, 499. [DOI], [ADS] (Cited on page 45.)

Kiraga, M. and Stẹpień, K., 2007, "Age-Rotation-Activity Relations for M Dwarf Stars", Acta Astron. 57, 149-172. [ADS], [arXiv:0707.2577] (Cited on page 45.)

Koch, R.H. and Pfeiffer, R.J., 1976, "The linear polarization of BY Draconis", Astrophys. J. Lett., 204, L47-L49. [DOI], [ADS] (Cited on page 23.)

Kochukhov, O. and Piskunov, N.E., 2002, "Doppler Imaging of stellar magnetic fields. II. Numerical experiments", Astron. Astrophys., 388, 868-888. [DOI], [ADS] (Cited on pages 16, 17, 19, and 21.)

Kochukhov, O., Drake, N.A., Piskunov, N.E. and de la Reza, R., 2004, "Multi-element abundance Doppler imaging of the rapidly oscillating Ap star HR 3831", Astron. Astrophys., 424, 935-950. [DOI], [ADS] (Cited on page 18.)

Kochukhov, O., Heiter, U., Piskunov, N.E., Ryde, N., Gustafsson, B., Bagnulo, S. and Plez, B., 2009, "Magnetic fields in M dwarf stars from high-resolution infrared spectra", in Cool Stars, Stellar Systems and the Sun: 15th Cambridge Workshop, St. Andrews, Scotland, 21 - 25 July 2008, (Ed.) Stempels, E., vol. 1094 of AIP Conference Proceedings, pp. 124-129, American Institute of Physics, Melville, NY. [DOI], [ADS] (Cited on pages 32 and 37.)

Kochukhov, O., Makaganiuk, V. and Piskunov, N.E., 2010, "Least-squares deconvolution of the stellar intensity and polarization spectra", Astron. Astrophys., 524, A5. [DOI], [ADS], [arXiv:1008.5115 [astroph.SR]] (Cited on page 16.) 
Kochukhov, O., Makaganiuk, V., Piskunov, N.E., Snik, F., Jeffers, S.V., Johns-Krull, C.M., Keller, C.U., Rodenhuis, M. and Valenti, J.A., 2011, "First Detection of Linear Polarization in the Line Profiles of Active Cool Stars", Astrophys. J. Lett., 732, L19. [DOI], [ADS], [arXiv:1103.6028 [astro-ph.SR]] (Cited on pages 12,41 , and 43.$)$

Kolenberg, K. and Bagnulo, S., 2009, "Observational constraints on the magnetic field of RR Lyrae stars", Astron. Astrophys., 498, 543-550. [DOI], [ADS] (Cited on page 23.)

Konstantinova-Antova, R., Aurière, M., Iliev, I.K., Cabanac, R., Donati, J.-F., Mouillet, D. and Petit, P., 2008, "Direct detection of a magnetic field at the surface of V390 Aurigae - an effectively single active giant", Astron. Astrophys., 480, 475-479. [DOI], [ADS] (Cited on page 44.)

Konstantinova-Antova, R., Aurière, M., Charbonnel, C., Drake, N.A., Schröder, K.-P., Stateva, I., Alecian, E., Petit, P. and Cabanac, R., 2010, "Direct detection of a magnetic field in the photosphere of the single $\mathrm{M}$ giant EK Bootis. How common is magnetic activity among M giants?", Astron. Astrophys., 524, A57. [DOI], [ADS], [arXiv:1009.2001 [astro-ph.SR]] (Cited on page 44.)

Landi Degl'Innocenti, E., 1982, "On the effective Landé factor of magnetic lines", Solar Phys., 77, 285-289. [DOI], [ADS] (Cited on page 10.)

Landi Degl'Innocenti, E. and Landolfi, M. (Eds.), 2004, Polarization in Spectral Lines, vol. 307 of Astrophysics and Space Science Library, Kluwer, Dordrecht. [ADS], [Google Books] (Cited on page 9.)

Landstreet, J.D., 1992, "Magnetic fields at the surfaces of stars", Astron. Astrophys. Rev., 4, 35-77. [DOI], [ADS] (Cited on page 9.)

Leroy, J.L., 1962, "Contributions à l'étude de la polarisation de la lumière solaire", Ann. Astrophys., 25, 127. [ADS] (Cited on pages 18 and 23.)

Linsky, J.L., Andrulis, C., Saar, S.H., Ayres, T.R. and Giampapa, M.S., 1994, "The Relationship Between Radiative and Magnetic Fluxes on Three Active Solar-type Dwarfs", in Cool Stars, Stellar Systems, and the Sun: Eight Cambridge Workshop, Athens, Georgia, October 11-14, 1993, (Ed.) Caillault, J.-P., vol. 64 of ASP Conference Series, pp. 438-440, Astronomical Society of the Pacific, San Francisco. [ADS] (Cited on page 36.)

Lites, B.W., 2000, "Remote sensing of solar magnetic fields", Rev. Geophys., 38, 1-36. [DOI], [ADS] (Cited on page 12.)

Marcy, G.W., 1981, "The magnetic field on the late-type dwarf XI Bootis A", Astrophys. J., 245, 624-629. [DOI], [ADS] (Cited on page 40.)

Marcy, G.W., 1984, "Observations of magnetic fields on solar-type stars", Astrophys. J., 276, $286-304$. [DOI], [ADS] (Cited on page 40.)

Marcy, G.W. and Basri, G., 1989, "Physical realism in the analysis of stellar magnetic fields. II. K dwarfs", Astrophys. J., 345, 480-488. [DOI], [ADS] (Cited on page 36.)

Marcy, G.W. and Bruning, D.H., 1984, "Magnetic field observations of evolved stars", Astrophys. J., 281, 286-291. [DOI], [ADS] (Cited on page 40.)

Marsden, S.C., Donati, J.-F., Semel, M., Petit, P. and Carter, B.D., 2006a, "Surface differential rotation and photospheric magnetic field of the young solar-type star HD 171488 (V889 Her)", Mon. Not. R. Astron. Soc., 370, 468-476. [DOI], [ADS] (Cited on page 43.)

Marsden, S.C., Mengel, M.W., Donati, J.-F., Carter, B.D., Semel, M. and Petit, P., 2006b, "The Magnetic Activity of Young Suns: Magnetic Topologies and Differential Rotation of F- and G-Dwarfs", in Solar Polarization 4, Proceedings of a meeting held in Boulder, Colorado, 19-23 September 2005, (Eds.) Casini, R., Lites, B.W., vol. 358 of ASP Conference Series, p. 401, Astronomical Society of the Pacific, San Francisco. [ADS] (Cited on page 43.) 
Martínez González, M.J., Asensio Ramos, A., Carroll, T.A., Kopf, M., Ramírez Vélez, J.C. and Semel, M., 2008, "PCA detection and denoising of Zeeman signatures in polarised stellar spectra", Astron. Astrophys., 486, 637-646. [DOI], [ADS], [arXiv:0804.4692] (Cited on page 22.)

Mathys, G. and Solanki, S.K., 1989, "Magnetic fields in late-type dwarfs: Preliminary results from a multiline approach", Astron. Astrophys., 208, 189-197. [ADS] (Cited on page 40.)

Mestel, L. and Landstreet, J.D., 2005, "Stellar Magnetic Fields", in Cosmic Magnetic Fields, (Eds.) Wielebinski, R., Beck, R., vol. 664 of Lecture Notes in Physics, pp. 183-218, Springer, Berlin; New York. [DOI], [ADS], [Google Books] (Cited on page 9.)

Meyer, F. and Meyer-Hofmeister, E., 1999, "On the source of viscosity in cool binary accretion disks", Astron. Astrophys., 341, L23-L26. [ADS], [arXiv:astro-ph/9901232] (Cited on page 48.)

Mohanty, S., Basri, G., Shu, F., Allard, F. and Chabrier, G., 2002, "Activity in Very Cool Stars: Magnetic Dissipation in Late M and L Dwarf Atmospheres", Astrophys. J., 571, 469-486. [DOI], [ADS], [arXiv:astro-ph/0201518] (Cited on page 48.)

Morin, J., Donati, J.-F., Petit, P., Delfosse, X., Forveille, T., Albert, L., Aurière, M., Cabanac, R., Dintrans, B., Fares, R., Gastine, T., Jardine, M.M., Lignières, F., Paletou, F., Ramírez Vélez, J.C. and Théado, S., 2008, "Large-scale magnetic topologies of mid M dwarfs", Mon. Not. R. Astron. Soc., 390, 567-581. [DOI], [ADS], [arXiv:0808.1423] (Cited on pages 22, 41, and 43.)

Morin, J., Donati, J.-F., Petit, P., Delfosse, X., Forveille, T. and Jardine, M.M., 2010, "Large-scale magnetic topologies of late M dwarfs", Mon. Not. R. Astron. Soc., 407, 2269-2286. [DOI], [ADS], [arXiv:1005.5552 [astro-ph.SR]] (Cited on pages 41, 43, and 53.)

Moutou, C., Donati, J.-F., Savalle, R., Hussain, G.A.J., Alecian, E., Bouchy, F., Catala, C., Collier Cameron, A., Udry, S. and Vidal-Madjar, A., 2007, "Spectropolarimetric observations of the transiting planetary system of the K dwarf HD 189733", Astron. Astrophys., 473, 651-660. [DOI], [ADS], [arXiv:0707.2016] (Cited on page 43.)

Mullan, D.J. and Bell, R.A., 1976, "Possible evidence for the occurrence of magnetic fields of order 10 kilogauss in the red dwarf star by Draconis", Astrophys. J., 204, 818-829. [DOI], [ADS] (Cited on pages 18 and 23.)

Narain, U. and Ulmschneider, P., 1996, "Chromospheric and Coronal Heating Mechanisms II", Space Sci. Rev., 75, 453-509. [DOI], [ADS] (Cited on page 9.)

Noyes, R.W., Hartmann, L.W., Baliunas, S.L., Duncan, D.K. and Vaughan, A.H., 1984, "Rotation, convection, and magnetic activity in lower main-sequence stars", Astrophys. J., 279, 763-777. [DOI], [ADS] (Cited on pages 45 and 50.)

Önehag, A., Heiter, U., Gustafsson, B., Piskunov, N.E., Plez, B. and Reiners, A., 2011, "M-dwarf metallicities - A high-resolution spectroscopic study in the near infrared", Astron. Astrophys., accepted. [ADS], [arXiv:1112.0141 [astro-ph.SR]] (Cited on page 32.)

Ortiz, A., Solanki, S.K., Domingo, V., Fligge, M. and Sanahuja, B., 2002, "On the intensity contrast of solar photospheric faculae and network elements", Astron. Astrophys., 388, 1036-1047. [DOI], [ADS], [arXiv:astro-ph/0207008] (Cited on page 24.)

Ossendrijver, M.A.J.H., 2003, "The solar dynamo", Astron. Astrophys. Rev., 11, 287-367. [DOI], [ADS] (Cited on page 45.)

Petit, P., Donati, J.-F., Oliveira, J.M., Aurière, M., Bagnulo, S., Landstreet, J.D., Lignières, F., Lüftinger, T., Marsden, S.C., Mouillet, D., Paletou, F., Strasser, S., Toqué, N. and Wade, G.A., 2004, "Photospheric magnetic field and surface differential rotation of the FK Com star HD 199178", Mon. Not. R. Astron. Soc., 351, 826-844. [DOI], [ADS], [arXiv:astro-ph/0403284] (Cited on pages 42 and 44.) 
Petit, P., Dintrans, B., Solanki, S.K., Donati, J.-F., Aurière, M., Lignières, F., Morin, J., Paletou, F., Ramírez Vélez, J.C., Catala, C. and Fares, R., 2008, "Toroidal versus poloidal magnetic fields in Sunlike stars: a rotation threshold", Mon. Not. R. Astron. Soc., 388, 80-88. [DOI], [ADS], [arXiv:0804.1290] (Cited on page 43.)

Pevtsov, A.A., Fisher, G.H., Acton, L.W., Longcope, D.W., Johns-Krull, C.M., Kankelborg, C.C. and Metcalf, T.R., 2003, "The Relationship Between X-Ray Radiance and Magnetic Flux", Astrophys. J., 598, 1387-1391. [DOI], [ADS] (Cited on pages 9, 25, and 26.)

Phan-Bao, N., Martín, E.L., Donati, J.-F. and Lim, J., 2006, "Magnetic Fields in M Dwarfs: Rapid Magnetic Field Variability in EV Lacertae", Astrophys. J. Lett., 646, L73-L76. [DOI], [ADS], [arXiv:astroph/0603480] (Cited on page 43.)

Phan-Bao, N., Lim, J., Donati, J.-F., Johns-Krull, C.M. and Martín, E.L., 2009, "Magnetic Field Topology in Low-Mass Stars: Spectropolarimetric Observations of M Dwarfs", Astrophys. J., 704, 1721-1729. [DOI], [ADS], [arXiv:0909.2355 [astro-ph.SR]] (Cited on pages 37 and 43.)

Piskunov, N.E. and Kochukhov, O., 2002, "Doppler Imaging of stellar magnetic fields. I. Techniques", Astron. Astrophys., 381, 736-756. [DOI], [ADS] (Cited on pages 12 and 19.)

Pizzolato, N., Maggio, A., Micela, G., Sciortino, S. and Ventura, P., 2003, "The stellar activity-rotation relationship revisited: Dependence of saturated and non-saturated X-ray emission regimes on stellar mass for late-type dwarfs", Astron. Astrophys., 397, 147-157. [DOI], [ADS] (Cited on pages 45 and 46.)

Preston, G.W., 1971, "The Mean Surface Fields of Magnetic Stars", Astrophys. J., 164, 309. [DOI], [ADS] (Cited on page 27.)

Reader, J. and Sugar, J., 1975, "Energy levels of iron, Fe I through Fe XXVI", J. Phys. Chem. Ref. Data, 4, 353-440. [DOI], [ADS] (Cited on page 10.)

Reiners, A., 2007, "The narrowest M-dwarf line profiles and the rotation-activity connection at very slow rotation", Astron. Astrophys., 467, 259-268. [DOI], [ADS], [arXiv:astro-ph/0702634] (Cited on pages 31 and 46.)

Reiners, A. and Basri, G., 2006, "Measuring Magnetic Fields in Ultracool Stars and Brown Dwarfs", Astrophys. J., 644, 497-509. [DOI], [ADS], [arXiv:astro-ph/0602221] (Cited on pages 32 and 33.)

Reiners, A. and Basri, G., 2007, "The First Direct Measurements of Surface Magnetic Fields on Very Low Mass Stars", Astrophys. J., 656, 1121-1135. [DOI], [ADS], [arXiv:astro-ph/0610365] (Cited on pages $25,32,33,37,38$, and 49.)

Reiners, A. and Basri, G., 2008, "Chromospheric Activity, Rotation, and Rotational Braking in M and L Dwarfs", Astrophys. J., 684, 1390-1403. [DOI], [ADS], [arXiv:0805.1059] (Cited on pages 48 and 54.)

Reiners, A. and Basri, G., 2009, "On the magnetic topology of partially and fully convective stars", Astron. Astrophys., 496, 787-790. [DOI], [ADS], [arXiv:0901.1659 [astro-ph.SR]] (Cited on pages 37, 54, and 55.)

Reiners, A. and Basri, G., 2010, "A Volume-Limited Sample of 63 M7-M9.5 Dwarfs. II. Activity, Magnetism, and the Fade of the Rotation-Dominated Dynamo", Astrophys. J., 710, 924-935. [DOI], [ADS], [arXiv:0912.4259 [astro-ph.SR]] (Cited on pages 25, 37, 38, 47, 48, 49, and 59.)

Reiners, A. and Christensen, U.R., 2010, "A magnetic field evolution scenario for brown dwarfs and giant planets", Astron. Astrophys., 522, A13. [DOI], [ADS], [arXiv:1007.1514 [astro-ph.EP]] (Cited on page 34.)

Reiners, A. and Mohanty, S., 2011, "Radius-Dependent Angular Momentum Evolution in Low-Mass Stars. I", Astrophys. J., 746, 43. [DOI], [ADS], [arXiv:1111.7071 [astro-ph.SR]] (Cited on page 54.)

Reiners, A. and Schmitt, J.H.M.M., 2003a, "Rotation and differential rotation in field F- and G-type stars", Astron. Astrophys., 398, 647-661. [DOI], [ADS] (Cited on page 22.) 
Reiners, A. and Schmitt, J.H.M.M., 2003b, "Differential rotation in rapidly rotating F-stars", Astron. Astrophys., 412, 813-819. [DOI], [ADS], [arXiv:astro-ph/0309616] (Cited on page 22.)

Reiners, A., Schmitt, J.H.M.M. and Liefke, C., 2007, "Rapid magnetic flux variability on the flare star CN Leonis", Astron. Astrophys., 466, L13-L16. [DOI], [ADS], [arXiv:astro-ph/0703172] (Cited on page 37.)

Reiners, A., Basri, G. and Browning, M., 2009a, "Evidence for Magnetic Flux Saturation in Rapidly Rotating M Stars", Astrophys. J., 692, 538-545. [DOI], [ADS], [arXiv:0810.5139] (Cited on pages 37, 46, and 47.)

Reiners, A., Basri, G. and Christensen, U.R., 2009b, "Surprisingly Weak Magnetism on Young Accreting Brown Dwarfs", Astrophys. J., 697, 373-379. [DOI], [ADS], [arXiv:0903.0857 [astro-ph.SR]] (Cited on pages $33,34,39$, and 59.)

Reiners, A., Joshi, N. and Goldman, B., 2012, "A catalogue of rotation and activity in early-M stars", Astron. J., accepted. [ADS], [1201.5774 [astro-ph.SR]] (Cited on page 54.)

Robinson, R.D., Worden, S.P. and Harvey, J.W., 1980, "Observations of magnetic fields on two late-type dwarf stars", Astrophys. J. Lett., 236, L155-L158. [DOI], [ADS] (Cited on page 40.)

Robinson Jr, R.D., 1980, "Magnetic field measurements on stellar sources: A new method", Astrophys. J., 239, 961-967. [DOI], [ADS] (Cited on pages 27 and 29.)

Rüedi, I., Solanki, S.K., Mathys, G. and Saar, S.H., 1997, "Magnetic field measurements on moderately active cool dwarfs", Astron. Astrophys., 318, 429-442. [ADS] (Cited on page 36.)

Saar, S.H., 1988, "Improved methods for the measurement and analysis of stellar magnetic fields", Astrophys. J., 324, 441-465. [DOI], [ADS] (Cited on pages 9, 17, and 28.)

Saar, S.H., 1990, "Magnetic fields on solar-like stars: The first decade", in Solar Photosphere: Structure, Convection, and Magnetic Fields, Kiev, USSR, May 15-20, 1989, (Ed.) Stenflo, J.O., vol. 138 of IAU Symposia, pp. 427-441, Kluwer Academic, Dordrecht. [ADS], [Google Books] (Cited on pages 40 and 51.)

Saar, S.H., 1994, "New Infrared Measurements of Magnetic Fields on Cool Stars", in Infrared Solar Physics, Proceedings of the 154th Symposium of the International Astronomical Union, held in Tucson, Arizona, U.S.A., March 2-6, 1992, (Eds.) Rabin, D.M., Jefferies, J.T., Lindsey, C., vol. 154 of IAU Symposia, p. 493, Kluwer Academic, Dordrecht; Boston. [ADS] (Cited on pages 31, 36, and 37.)

Saar, S.H., 1996a, "Recent magnetic fields measurements of stellar", in Stellar Surface Structure, Proceedings of the 176th Symposium of the International Astronomical Union, 9-13 October 1995, Vienna, Austria, (Eds.) Strassmeier, K.G., Linsky, J.L., vol. 176 of IAU Symposia, p. 237, Kluwer, Dordrecht; Boston. [ADS], [Google Books] (Cited on pages 46 and 47.)

Saar, S.H., 1996b, "Recent Measurements of Stellar Magnetic Fields", in Magnetodynamic Phenomena in the Solar Atmosphere: Prototypes of Stellar Magnetic Activity, Proceedings of the 153rd Colloquium of the IAU, held in Makuhari, Japan, $22-27$ May 1995, (Eds.) Uchida, Y., Kosugi, T., Hudson, H.S., pp. 367-374, Kluwer Academics, Dordrecht. [ADS], [Google Books] (Cited on pages 27, 29, 36, and 37.)

Saar, S.H., 2001, "Recent Measurements of (and Inferences About) Magnetic Fields on K and M Stars", in Cool Stars, Stellar Systems and the Sun: Eleventh Cambridge Workshop, Puerto de la Cruz, Tenerife, Spain, October 4-8, 1999, (Eds.) García López, R.J., Rebolo, R., Zapatero Osorio, M.R., vol. 223 of ASP Conference Series, p. 292, Astronomical Society of the Pacific, San Francisco. [ADS] (Cited on pages $25,29,46$, and 47.$)$

Saar, S.H. and Baliunas, S.L., 1992, "The Magnetic Cycle of Kappa Ceti", in The Solar Cycle, Proceedings of the NSO/Sacramento Peak 12th Summer Workshop, 15-18 October 1991, (Ed.) Harvey, K.L., vol. 27 of ASP Conference Series, pp. 197-202, Astronomical Society of the Pacific, San Francisco. [ADS] (Cited on page 36.$)$ 
Saar, S.H. and Huovelin, J., 1993, "Broad-band linear polarization in cool stars. II. Amplitude and wavelength dependence for magnetic and scattering regions", Astrophys. J., 404, 739-750. [DOI], [ADS] (Cited on page 23.)

Saar, S.H. and Linsky, J.L., 1985, "The photospheric magnetic field of the dM3.5e flare star AD Leonis", Astrophys. J. Lett., 299, L47-L50. [DOI], [ADS] (Cited on pages 31, 37, and 51.)

Saar, S.H. and Solanki, S.K.., 1992, "How Accurate are Stellar Magnetic Field Measurements?", in Cool Stars, Stellar Systems, and the Sun: Seventh Cambridge Workshop, Tuscon, Arizona, October 9-12, 1991, (Eds.) Giampapa, M.S., Bookbinder, J.A., vol. 26 of ASP Conference Series, pp. 259-261, Astronomical Society of the Pacific, San Francisco. [ADS] (Cited on page 28.)

Saar, S.H., Linsky, J.L. and Beckers, J.M., 1986, "The magnetic field of the BY Draconis flare star EQ Virginis", Astrophys. J., 302, 777-784. [DOI], [ADS] (Cited on page 40.)

Saar, S.H., Piskunov, N.E. and Tuominen, I., 1992, "Magnetic Surface Images of the BY DRA Star HD 82558", in Cool Stars, Stellar Systems, and the Sun: Seventh Cambridge Workshop, Tuscon, Arizona, October 9-12, 1991, (Eds.) Giampapa, M.S., Bookbinder, J.A., vol. 26 of ASP Conference Series, pp. 255-258, Astronomical Society of the Pacific, San Francisco. [ADS] (Cited on page 18.)

Saar, S.H., Bünte, M. and Solanki, S.K., 1994a, "How Accurate Are Stellar Magnetic Field Measurements? II. Analysis of Disk-Integrated Flux Tube Models", in Cool Stars, Stellar Systems, and the Sun: Eighth Cambridge Workshop, Athens, Georgia, October 11-14, 1993, (Ed.) Caillault, J.-P., vol. 64 of ASP Conference Series, pp. 474-476, Astronomical Society of the Pacific, San Francisco. [ADS] (Cited on page 28.)

Saar, S.H., Martens, P.C.H., Huovelin, J. and Linnaluoto, S., 1994b, "Possible detection of a stellar flare-generated particle beam in polarized light", Astron. Astrophys., 286, 194-202. [ADS] (Cited on page 23.)

Saar, S.H., Piskunov, N.E. and Tuominen, I., 1994c, "Multiepoch Magnetic Surface Images of LQ Hya", in Cool Stars, Stellar Systems, and the Sun: Eighth Cambridge Workshop, Athens, Georgia, October 11-14, 1993, (Ed.) Caillault, J.-P., vol. 64 of ASP Conference Series, pp. 661-663, Astronomical Society of the Pacific, San Francisco. [ADS] (Cited on page 18.)

Schrijver, C.J., 1990, "Relations between the photospheric magnetic field and the emission from the outer atmospheres of cool stars. II. The C IV $1550 \AA$ doublet", Astron. Astrophys., 234, 315-322. [ADS] (Cited on page 25.)

Schrijver, C.J. and Title, A.M., 2011, "Long-range magnetic couplings between solar flares and coronal mass ejections observed by SDO and STEREO", J. Geophys. Res., 116, A04108. [DOI], [ADS] (Cited on page 52.)

Schrijver, C.J., Coté, J., Zwaan, C. and Saar, S.H., 1989, "Relations between the photospheric magnetic field and the emission from the outer atmospheres of cool stars. I. The solar Ca II K line core emission", Astrophys. J., 337, 964-976. [DOI], [ADS] (Cited on pages 9, 24, and 25.)

Semel, M., 1989, "Zeeman-Doppler imaging of active stars. I. Basic principles", Astron. Astrophys., 225, 456-466. [ADS] (Cited on pages 18 and 22.)

Sennhauser, C. and Berdyugina, S.V., 2010, "Zeeman component decomposition for recovering common profiles and magnetic fields", Astron. Astrophys., 522, A57. [DOI], [ADS] (Cited on pages 22 and 23.)

Sennhauser, C. and Berdyugina, S.V., 2011, "First detection of a weak magnetic field on the giant Arcturus: remnants of a solar dynamo?", Astron. Astrophys., 529, A100. [DOI], [ADS] (Cited on page 44.)

Shulyak, D., Reiners, A., Wende, S., Kochukhov, O., Piskunov, N.E. and Seifahrt, A., 2010, "Modelling the molecular Zeeman effect in M-dwarfs: methods and first results", Astron. Astrophys., 523, A37. [DOI], [ADS], [arXiv:1008.2512 [astro-ph.SR]] (Cited on page 32.) 
Shulyak, D., Seifahrt, A., Reiners, A., Kochukhov, O. and Piskunov, N.E., 2011, "Rotation, magnetism and metallicity of M dwarf systems", Mon. Not. R. Astron. Soc., 418, 2548-2557. [DOI], [ADS], [arXiv:1108.3465 [astro-ph.SR]] (Cited on page 32.)

Skelly, M.B., Donati, J.-F., Bouvier, J., Grankin, K.N., Unruh, Y.C., Artemenko, S.A. and Petrov, P.P., 2010, "Dynamo processes in the T Tauri star V410 Tau", Mon. Not. R. Astron. Soc., 403, 159-169. [DOI], [ADS], [arXiv:0911.5476 [astro-ph.SR]] (Cited on page 44.)

Solanki, S.K. and Stenflo, J.O., 1985, "Models of solar magnetic fluxtubes: Constraints imposed by Fe I and II lines", Astron. Astrophys., 148, 123-132. [ADS] (Cited on page 10.)

Solanki, S.K., Inhester, B. and Schüssler, M., 2006, "The solar magnetic field", Rep. Prog. Phys., 69, 563-668. [DOI], [ADS], [arXiv:1008.0771 [astro-ph.SR]] (Cited on page 7.)

Spruit, H.C. and Zweibel, E.G., 1979, "Convective instability of thin flux tubes", Solar Phys., 62, 15-22. [DOI], [ADS] (Cited on page 51.)

Stenflo, J.O., 1994, Solar Magnetic Fields: Polarization Radiation Diagnostics, vol. 189 of Astrophysics and Space Science Library, Kluwer Academic, Dordrecht. [Google Books] (Cited on page 22.)

Stokes, G.G., 1852, "On the composition and resolution of streams of polarized light from different sources", Trans. Cambridge Philos. Soc., 9, 399-416 (Cited on page 11.)

Strassmeier, K.G., 2002, "Doppler images of starspots", Astron. Nachr., 323, 309-316. [DOI], [ADS] (Cited on page 18.)

Strassmeier, K.G., Pallavicini, R., Rice, J.B., Andersen, M.I. and Zerbi, F.M., 2004, "The science case of the PEPSI high-resolution echelle spectrograph and polarimeter for the LBT", Astron. Nachr., 325, 278-298. [DOI], [ADS] (Cited on page 19.)

Tinbergen, J., 1996, Astronomical Polarimetry, Cambridge University Press, Cambridge; New York. [ADS], [Google Books] (Cited on page 11.)

Tinbergen, J. and Zwaan, C., 1981, "Magnetic structure in cool stars. II. Observational evidence for transverse magnetic fields", Astron. Astrophys., 101, 223-225. [ADS] (Cited on page 23.)

Trujillo Bueno, J., 2006, "A Gentle Introduction to the Physics of Spectral Line Polarization", in Solar Physics and Solar Eclipses (SPSE 2006), Proceedings of an International Symposium held at Waw an Namos, Libya, 27 - 29 March 2006, (Eds.) Ramelli, R., Shalabiea, O., Saleh, I., Stenflo, J.O., pp. 77-92, Istituto Ricerche Solari Locarno, Locarno. [ADS]. URL (accessed 23 June 2011): http://www.irsol.ch/spse/ (Cited on page 9.)

Trujillo Bueno, J., Shchukina, N. and Asensio Ramos, A., 2004, "A substantial amount of hidden magnetic energy in the quiet Sun", Nature, 430, 326-329. [DOI], [ADS], [arXiv:astro-ph/0409004] (Cited on pages 7 and 9.)

Unno, W., 1956, "Line Formation of a Normal Zeeman Triplet", Publ. Astron. Soc. Japan, 8, 108-125. [ADS] (Cited on page 22.)

Valenti, J.A., Marcy, G.W. and Basri, G., 1995, "Infrared Zeeman analysis of $\epsilon$ Eridani", Astrophys. J., 439, 939-956. [DOI], [ADS] (Cited on pages 17, 28, 29, and 36.)

Valenti, J.A., Piskunov, N.E. and Johns-Krull, C.M., 1998, "Spectral Synthesis of TiO Lines", Astrophys. J., 498, 851. [DOI], [ADS] (Cited on page 32.)

Valenti, J.A., Johns-Krull, C.M. and Piskunov, N.E., 2001, "Using FeH to Measure Magnetic Fields on Cool Stars and Brown Dwarfs", in Cool Stars, Stellar Systems and the Sun: Eleventh Cambridge Workshop, Puerto de la Cruz, Tenerife, Spain, October 4-8, 1999, (Eds.) García López, R.J., Rebolo, R., Zapatero Osorio, M.R., vol. 223 of ASP Conference Series, p. 1579, Astronomical Society of the Pacific, San Francisco. [ADS] (Cited on page 32.) 
Vogt, S.S., 1980, "A magnetic study of spotted UV Ceti flare stars and related late-type dwarfs", Astrophys. J., 240, 567-584. [DOI], [ADS] (Cited on pages 27 and 40.)

Vogt, S.S. and Penrod, G.D., 1983, "Doppler imaging of spotted stars: Application to the RS Canum Venaticorum star HR 1099", Publ. Astron. Soc. Pac., 95, 565-576. [DOI], [ADS] (Cited on page 18.)

Wade, G.A., Donati, J.-F., Landstreet, J.D. and Shorlin, S.L.S., 2000, "Spectropolarimetric measurements of magnetic Ap and Bp stars in all four Stokes parameters", Mon. Not. R. Astron. Soc., 313, 823-850. [DOI], [ADS] (Cited on pages 12 and 22.)

Wallace, L., Livingston, W.C., Bernath, P.F. and Ram, R.S., 1999, An Atlas of the Sunspot Umbral Spectrum in the Red and Infrared from 8900 to $15,050 \mathrm{~cm}^{-1}$ (6642 to 11,230 A), revised, NSO Technical Report \#99-001, National Solar Observatory, Tucson, AZ. [ADS]. Online version (accessed 7 February 2012):

ftp://nsokp.nso.edu/pub/atlas/ (Cited on page 32.)

Wende, S., Reiners, A. and Ludwig, H.-G., 2009, "3D simulations of M star atmosphere velocities and their influence on molecular FeH lines", Astron. Astrophys., 508, 1429-1442. [DOI], [ADS], [arXiv:0910.3493 [astro-ph.SR]] (Cited on page 32.)

Wende, S., Reiners, A., Seifahrt, A. and Bernath, P.F., 2010, "CRIRES spectroscopy and empirical lineby-line identification of $\mathrm{FeH}$ molecular absorption in an $\mathrm{M}$ dwarf", Astron. Astrophys., 523, A58. [DOI], [ADS], [arXiv:1007.4116 [astro-ph.SR]] (Cited on page 32.)

West, A.A., Hawley, S.L., Bochanski, J.J., Covey, K.R., Reid, I.N., Dhital, S., Hilton, E.J. and Masuda, M., 2008, "Constraining the Age-Activity Relation for Cool Stars: The Sloan Digital Sky Survey Data Release 5 Low-Mass Star Spectroscopic Sample", Astron. J., 135, 785-795. [DOI], [ADS], [arXiv:0712.1590] (Cited on page 54.)

Yang, H. and Johns-Krull, C.M., 2011, "Magnetic Field Measurements of T Tauri Stars in the Orion Nebula Cluster", Astrophys. J., 729, 83. [DOI], [ADS], [arXiv:1102.3662 [astro-ph.SR]] (Cited on pages 34, 39, and 59.)

Yang, H., Johns-Krull, C.M. and Valenti, J.A., 2007, "Spectropolarimetry of the Classical T Tauri Star TW Hydrae", Astron. J., 133, 73-80. [DOI], [ADS], [arXiv:astro-ph/0609289] (Cited on page 44.)

Yang, H., Johns-Krull, C.M. and Valenti, J.A., 2008, "Magnetic Properties of Young Stars in the TW Hydrae Association", Astron. J., 136, 2286-2294. [DOI], [ADS], [arXiv:0809.3290] (Cited on page 39.)

Zeeman, P., 1897, "On the Influence of Magnetism on the Nature of the Light Emitted by a Substance", Astrophys. J., 5, 332. [DOI], [ADS] (Cited on page 9.) 